\title{
Allylic Azide Rearrangement in Tandem with Huisgen Cycloaddition for Stereoselective Annulation: Synthesis of C- Glycosyl Iminosugars
}

Lorna Moynihan, Rekha Chadda, Patrick McArdle and Paul V. Murphy*.

Email: paul.v.murphy@nuigalway.ie

Table of Contents

General Experimental Methods

Page S2

Experimental procedures/analytical data for compounds in the manuscript......Page S2-S20

Schemes for synthesis of compounds 24 and $\mathbf{3 1}$ ...Page S21

Experimental procedures/analytical data relevant for preparation of $\mathbf{2 4 / 3 1 . . . . . . P a g e ~ S 2 1 - S 3 0 ~}$

Table S1: ${ }^{1} \mathrm{H}-\mathrm{NMR}$ data for $20 \alpha$ and comparison with natural product. ..Page S31

Table S2: ${ }^{13}$ C-NMR data for $\mathbf{2 0} \alpha$ and comparison with natural product/20 $\beta$......Page S31

Table S3 and associated plot: Differences in ${ }^{13} \mathrm{C}-\mathrm{NMR}$ data for $\mathbf{2 0} \alpha$ from natural product $/ 20 \beta$. Page S32

Tables S4, S5 and associated plots: Differences in ${ }^{13} \mathrm{C}-\mathrm{NMR}$ data between $\mathbf{2 7}$ and $29 / 30$. Page S33-34

Table S6: ${ }^{1} \mathrm{H}-\mathrm{NMR}$ data for $\mathbf{2 7}$ and comparison with 29/30 Page S34

References: Page S35 


\section{Supporting information - Experimental Section}

\section{General Experimental Methods}

NMR spectra were recorded using a $500 \mathrm{MHz}$ spectrometer. Chemical shifts are reported relative to internal $\mathrm{Me}_{4} \mathrm{Si}$ in $\mathrm{CDCl}_{3}(\delta 0.0), \mathrm{HOD}$ for $\mathrm{D}_{2} \mathrm{O}(\delta 4.65)$ and $\mathrm{CD}_{2} \mathrm{HOD}(\delta 3.31)$ for ${ }^{1} \mathrm{H}$ and $\mathrm{CDCl}_{3}(\delta 77.16)$ and $\mathrm{CD}_{3} \mathrm{OD}(\delta 49.0)$ for ${ }^{13} \mathrm{C} .{ }^{1} \mathrm{H}-\mathrm{NMR}$ signals were assigned with the aid of COSY. ${ }^{13} \mathrm{C}$ signals were assigned with the aid of DEPT-135, HSQC and HMBC. Coupling constants $(J)$ are reported in Hertz and are reported uncorrected. Low and high resolution mass spectra were measured in positive and/or negative mode as indicated using $\mathrm{MeCN}, \mathrm{H}_{2} \mathrm{O}$ and/or $\mathrm{MeOH}$ as solvent using a Waters LCT Mass Spectrometry instrument. FT-IR spectra were recorded using a polarized UATR (Universal Attenuated Total Reflectance) Accessory. Optical rotations were determined at the sodium D line at $23{ }^{\circ} \mathrm{C}$ using $\mathrm{CHCl}_{3}$ or $\mathrm{D}_{2} \mathrm{O}$ as indicated. TLC was performed on aluminium sheets pre-coated with Silica Gel $60\left(\mathrm{HF}_{254}\right.$, E. Merck) and spots visualized by UV and charring with cerium (IV) molybdate solution or ninhydrin solutions. Flash column chromatography was generally employed and was carried out using silica gel $60(0.040-0.630 \mathrm{~mm})$ using a stepwise solvent polarity gradient correlated with TLC mobility. Chromatography solvents used were petroleum ether (40-60 ${ }^{\circ} \mathrm{C}$, Fisher Scientific), EtOAc, $\mathrm{CH}_{2} \mathrm{Cl}_{2}$ and $\mathrm{MeOH}$ (Sigma Aldrich). Ion exchange chromatography was carried out using DOWEX-50WX8 (200-400 mesh) resin. Anhydrous pyridine was used as purchased from Sigma-Aldrich. THF, toluene, $\mathrm{CH}_{2} \mathrm{Cl}_{2}$, $\mathrm{Et}_{2} \mathrm{O}, \mathrm{DMF}$ and methanol were used as obtained from a Pure-Solv ${ }^{\mathrm{TM}}$ solvent purification system. The numbering scheme for NMR assignments is indicated in some places below.
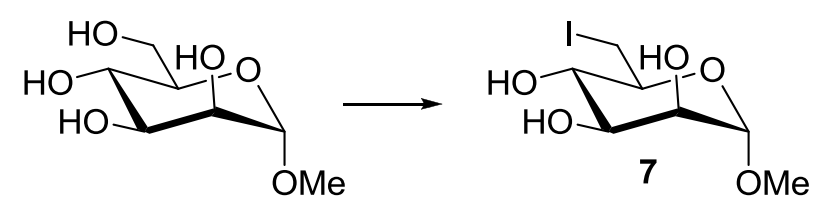

Methyl 6-deoxy-6-iodo- $\alpha$-D-mannopyranoside: Methyl $\alpha$-D-mannopyranoside $(10.0 \mathrm{~g}$, $0.051 \mathrm{~mol})$ was dissolved in THF (100 mL). Triphenyl phosphine (20.3 g, $0.077 \mathrm{~mol})$ and imidazole $(7.0 \mathrm{~g}, 0.103 \mathrm{~mol})$ were added and the mixture was heated to approx. $60{ }^{\circ} \mathrm{C}$. Iodine (19.6 g, $0.077 \mathrm{~mol})$ in THF was added portion-wise and the colour changed from brown to yellow. The reaction was stirred while heating at reflux for $4 \mathrm{~h}$. The salts were then filtered and solvent was removed under diminished pressure. Flash column chromatography of the residue $\left(\mathrm{CH}_{2} \mathrm{Cl}_{2}\right.$ followed by 1:9 $\left.\mathrm{MeOH}: \mathrm{CH}_{2} \mathrm{Cl}_{2}, \mathrm{R}_{\mathrm{f}} 0.15\right)$ gave $7(11.7 \mathrm{~g}, 75 \%)$ as a white solid. All analytical data provided below for corresponded well to previously reported data. ${ }^{1}$ $[\alpha]_{\mathrm{D}}+19.0\left(c 7.9, \mathrm{D}_{2} \mathrm{O}\right) ;{ }^{1} \mathrm{H} \mathrm{NMR}\left(500 \mathrm{MHz}, \mathrm{D}_{2} \mathrm{O}\right) \delta 4.53(\mathrm{~d}, J=1.7 \mathrm{~Hz}, 1 \mathrm{H}, \mathrm{H}-1), 3.74(\mathrm{dd}$, 


\section{Supporting information - Experimental Section}

$J=3.5,1.7 \mathrm{~Hz}, 1 \mathrm{H}, \mathrm{H}-2), 3.58$ (dd, $J=9.6,3.5 \mathrm{~Hz}, 1 \mathrm{H}, \mathrm{H}-3), 3.43$ - 3.35 (m, 2H, H-4, H-6, overlapping signals), $3.27-3.23(\mathrm{~m}, 1 \mathrm{H}, \mathrm{H}-5), 3.22\left(\mathrm{~s}, 3 \mathrm{H},-\mathrm{OCH}_{3}\right), 3.15$ (dd, $J=11.0,7.2$ $\mathrm{Hz}, 1 \mathrm{H}, \mathrm{H}-6) ;{ }^{13} \mathrm{C}$ NMR (126 MHz, D $\left.2 \mathrm{O}\right) \delta 101.0$ (C-1), 71.4 (C-5), 70.6 (C-4), 70.1 (C-3), 69.8 (C-2), $55.0\left(-\mathrm{OCH}_{3}\right), 6.5$ (C-6); FTIR 3113, 2976, 2937, 1988, 1576, 1525, 1412, 1327, 1256, 1187, 1130, 1085, 1041, 962, 917, 748, $657 \mathrm{~cm}^{-1}$; LRMS (ESI) $\mathrm{m} / z$ calc for $\mathrm{C}_{7} \mathrm{H}_{17} \mathrm{O}_{5} \mathrm{IN}: 322.0$, Found: $322.1\left[\mathrm{M}+\mathrm{NH}_{4}\right]^{+}$.

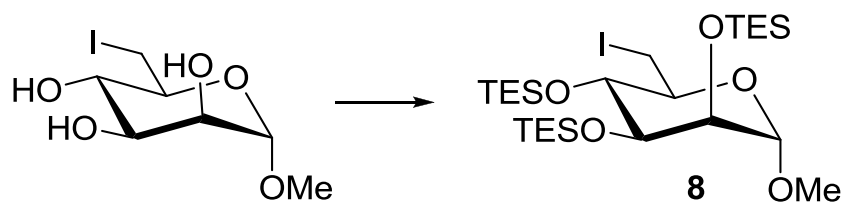

Methyl 6-deoxy-6-iodo-2,3,4-tri- $O$-triethylsilyl- $\alpha$-D-mannopyranoside 8 The precursor iodide 7 (16.0 g, $0.053 \mathrm{~mol})$ was suspended in $\mathrm{CH}_{2} \mathrm{Cl}_{2}(200 \mathrm{~mL})$. Imidazole (21.5 g, 0.317 mol) and chlorotriethylsilane $(53.0 \mathrm{~mL}, 0.317 \mathrm{~mol})$ were added and the reaction was stirred at room temp overnight. The reaction was diluted with $\mathrm{H}_{2} \mathrm{O}(100 \mathrm{~mL})$ and stirred for $15 \mathrm{~min}$. The layers were separated and the organic layer was washed with $\mathrm{H}_{2} \mathrm{O}(100 \mathrm{~mL})$, dried over $\mathrm{Na}_{2} \mathrm{SO}_{4}$ and the solvent was removed under diminished pressure. Flash column chromatography of the crude residue (hexane followed by 1:20 EtOAc-hexane, $R_{f} 0.6$ ) gave 8 as a clear oil $(23.8 \mathrm{~g}, 70 \%)$. All analytical data provided for the title compound corresponded well to previously reported data; ${ }^{1}[\alpha]_{\mathrm{D}}+35.4\left(c 0.81, \mathrm{CHCl}_{3}\right) ;{ }^{1} \mathrm{H} \mathrm{NMR}\left(500 \mathrm{MHz}, \mathrm{CDCl}_{3}\right) \delta$ 4.51 (apt s, 1H, H-1), $3.83-3.78$ (m, 3H, H-2, H-3, H-4), 3.56 (dd, $J=10.0,3.5 \mathrm{~Hz}, 1 \mathrm{H}, \mathrm{H}-$ 6), 3.41 (s, 3H, $-\mathrm{OCH}_{3}$ ), $3.32-3.25$ (m, 2H, H-5, H-6'), $1.00-0.94$ (m, 27H, $\left.-\mathrm{Si}\left(\mathrm{CH}_{2} \mathrm{CH}_{3}\right)_{3}\right)$, $0.69-0.59\left(\mathrm{~m}, 18 \mathrm{H},-\mathrm{Si}\left(\mathrm{CH}_{2} \mathrm{CH}_{3}\right)_{3}\right) ;{ }^{13} \mathrm{C} \mathrm{NMR}\left(126 \mathrm{MHz}, \mathrm{CDCl}_{3}\right): \delta 101.7(\mathrm{C}-1), 73.6-$ $72.5(\mathrm{CH}, \mathrm{C}-2, \mathrm{C}-3, \mathrm{C}-4, \mathrm{C}-5), 55.2\left(-\mathrm{OCH}_{3}\right), 8.3(\mathrm{C}-6), 7.1-6.8\left(-\mathrm{Si}\left(\mathrm{CH}_{2} \mathrm{CH}_{3}\right)_{3}\right), 5.2-5.1$ ($\left.\mathrm{Si}\left(\mathrm{CH}_{2} \mathrm{CH}_{3}\right)_{3}\right)$; FTIR 2954, 2911, 2877, 1459, 1413, 1378, 1238, 1135, 1102, 1062, 1003, 967, 919, 880, 837, 803, 782, $721 \mathrm{~cm}^{-1}$; HRMS (ESI) $\mathrm{m} / z$ calc for $\mathrm{C}_{27} \mathrm{H}_{58} \mathrm{O}_{5} \mathrm{Si}_{3} \mathrm{INNa}$ : 710.2565, Found: $710.2569[\mathrm{M}+\mathrm{MeCN}+\mathrm{Na}]^{+}$.
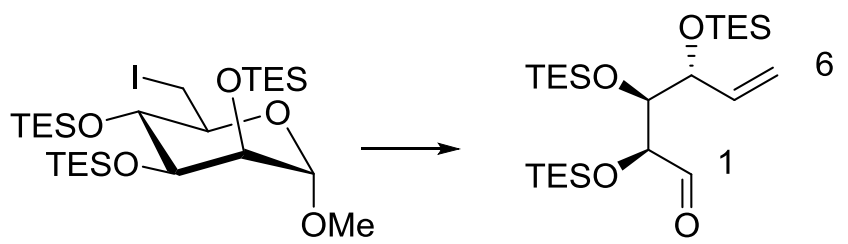

(2S,3S,4R)-5,6-Dideoxy-2,3,4-tri- $\boldsymbol{O}$-triethylsilyl-hex-5-en-1-al Compound 8 (3.1 g, 4.81 mmol) was dissolved in a mixture of THF- $\mathrm{H}_{2} \mathrm{O}(9: 1,50 \mathrm{~mL})$. Pre-activated $\mathrm{Zn}$ dust $(3.2 \mathrm{~g}$, $48.1 \mathrm{mmol}$ ) was added and the mixture was sonicated at $40{ }^{\circ} \mathrm{C}$ for $2-4 \mathrm{~h}$. The mixture was 


\section{Supporting information - Experimental Section}

filtered and washed with $\mathrm{Et}_{2} \mathrm{O}(50 \mathrm{~mL})$. The organic layer was washed with $\mathrm{H}_{2} \mathrm{O}(30 \mathrm{~mL})$, satd aq $\mathrm{NaHCO}_{3}(30 \mathrm{~mL})$ and brine $(30 \mathrm{~mL})$, dried over $\mathrm{Na}_{2} \mathrm{SO}_{4}$ and the solvent was removed to give the title aldehyde $(1.97 \mathrm{~g}, 84 \%)$ as a clear oil. The product was used immediately due to the risk of epimerization; $\left.[\alpha]_{\mathrm{D}}+33.4(c) 3.4, \mathrm{CHCl}_{3}\right) ;{ }^{1} \mathrm{H} \mathrm{NMR}\left(500 \mathrm{MHz}, \mathrm{CDCl}_{3}\right) \delta 9.52$ (d, $J=1.3 \mathrm{~Hz}, 1 \mathrm{H}, \mathrm{H}-1), 5.97$ (ddd, $J=17.0,10.4,6.3 \mathrm{~Hz}, 1 \mathrm{H}, \mathrm{H}-5), 5.23$ (dt, $J=17.4,1.6$ Hz, 1H, H-6), 5.14 (dt, $J=10.5,1.5 \mathrm{~Hz}, 1 \mathrm{H}, \mathrm{H}-6$ ') 4.18 (tt, $J=6.3,1.4 \mathrm{~Hz}, 1 \mathrm{H}, \mathrm{H}-4), 4.13$ (t, $J=1.4 \mathrm{~Hz}, 1 \mathrm{H}, \mathrm{H}-2), 3.93(\mathrm{dd}, J=6.2,1.5 \mathrm{~Hz}, 1 \mathrm{H}, \mathrm{H}-3), 1.00-0.90(\mathrm{~m}, 27 \mathrm{H}$, $\left.\mathrm{Si}\left(\mathrm{CH}_{2} \mathrm{CH}_{3}\right)_{3}\right), 0.66-0.53\left(\mathrm{~m}, 18 \mathrm{H},-\mathrm{Si}\left(\mathrm{CH}_{2} \mathrm{CH}_{3}\right)_{3}\right) ;{ }^{13} \mathrm{C} \mathrm{NMR}\left(126 \mathrm{MHz}, \mathrm{CDCl}_{3}\right) \delta 202.4$

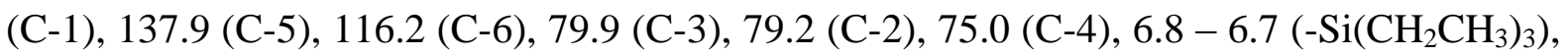
4.8 - $4.7\left(-\mathrm{Si}\left(\mathrm{CH}_{2} \mathrm{CH}_{3}\right)_{3}\right)$; FTIR 2955, 2912, 2878, 1734, 1459, 1414, 1379, 1239, 1135 , 1110, 1079, 1003, 974, 924, 813, $725 \mathrm{~cm}^{-1}$; HRMS (ESI) $\mathrm{m} / z$ calc for $\mathrm{C}_{24} \mathrm{H}_{52} \mathrm{O}_{4} \mathrm{Si}_{3} \mathrm{Na}$ : 511.3071, Found: $511.3095[\mathrm{M}+\mathrm{Na}]^{+}$.
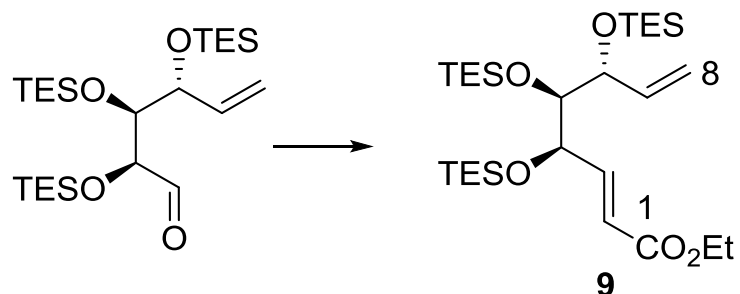

(4R,5S,6R,E)-Ethyl 4,5,6-tris(triethylsilyloxy)octa-2,7-dienoate 9 The precursor aldehyde $(2.4 \mathrm{~g}, \quad 0.049 \mathrm{~mol})$ was dissolved in toluene $(50 \mathrm{~mL})$. (Carbethoxy methylene)triphenylphosphorane $(2.5 \mathrm{~g}, 0.073 \mathrm{~mol})$ was added and the reaction was heated to reflux for $12 \mathrm{~h}$. The solvent was removed under diminished pressure. Flash column chromatography of the residue (hexane followed by 1:40 EtOAc-hexane, $\mathrm{R}_{\mathrm{f}} 0.5$ ) gave 9 (2.2 g, $80 \%)$ as a clear oil; $[\alpha]_{\mathrm{D}}+40.8\left(c 0.31, \mathrm{CHCl}_{3}\right) ;{ }^{1} \mathrm{H} \mathrm{NMR}\left(500 \mathrm{MHz}, \mathrm{CDCl}_{3}\right) \delta 6.98(\mathrm{dd}, J$ $=15.6,5.1 \mathrm{~Hz}, 1 \mathrm{H}, \mathrm{H}-3), 5.97-5.88$ (m, 2H, H-2, H-7, overlapping signals), 5.25 (dt, $J=$ $17.4,1.8 \mathrm{~Hz}, 1 \mathrm{H}, \mathrm{H}-8), 5.14$ (dt, $J=10.6,1.8 \mathrm{~Hz}, 1 \mathrm{H}, \mathrm{H}-8$ '), 4.48 (dt, $J=5.1,1.6 \mathrm{~Hz}, 1 \mathrm{H}, \mathrm{H}-$ 4), 4.18 (q, $J=7.1 \mathrm{~Hz}, 2 \mathrm{H},-\mathrm{OCH}_{2}$ ), 4.13 (q, $\left.J=5.4,4.7 \mathrm{~Hz}, 1 \mathrm{H}, \mathrm{H}-6\right), 3.80-3.78$ (m, 1H, $\mathrm{H}-5), 1.27\left(\mathrm{t}, J=7.1 \mathrm{~Hz}, 3 \mathrm{H},-\mathrm{CH}_{3}\right), 1.00-0.91\left(\mathrm{~m} 27 \mathrm{H},-\mathrm{Si}\left(\mathrm{CH}_{2} \mathrm{CH}_{3}\right)_{3}\right), 0.63-0.57(\mathrm{~m}$,

18H, $\left.-\mathrm{Si}\left(\mathrm{CH}_{2} \mathrm{CH}_{3}\right)_{3}\right) ;{ }^{13} \mathrm{C}$ NMR (126 MHz, $\left.\mathrm{CDCl}_{3}\right): \delta 166.7$ (C-1), 148.8 (C-3), $137.8(\mathrm{C}-2)$, 121.2 (C-7), 115.3 (C-8), 80.8 (C-5), 74.9 (C-6), 72.0 (C-4), $60.0\left(-\mathrm{OCH}_{2}\right), 14.2\left(-\mathrm{CH}_{3}\right), 6.83$ - $6.82\left(-\mathrm{Si}\left(\mathrm{CH}_{2} \mathrm{CH}_{3}\right)_{3}\right), 4.9-4.8\left(-\mathrm{Si}\left(\mathrm{CH}_{2} \mathrm{CH}_{3}\right)_{3}\right)$; FTIR 2955, 2912, 2878, 1724, 1660, 1459, 1414, 1367, 1262, 1239, 1113, 1064, 1004, 975, 923, 856, 798, $723 \mathrm{~cm}^{-1}$; HRMS (ESI) $\mathrm{m} / z$ calc for $\mathrm{C}_{28} \mathrm{H}_{58} \mathrm{O}_{5} \mathrm{Si}_{3} \mathrm{Na}$ : 581.3490, Found: 581.3477 [M+Na] ${ }^{+}$. 


\section{Supporting information - Experimental Section}

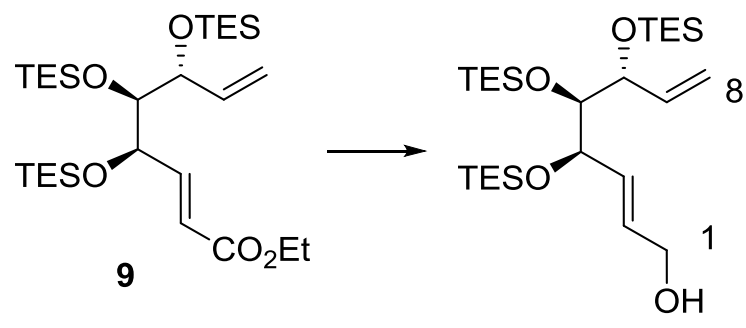

(4R,5S,6R,E)-4,5,6-Tris(triethylsilyloxy)octa-2,7-dien-1-ol Compound 9 (2.2 g, 3.87 mmol) was dissolved in $\mathrm{CH}_{2} \mathrm{Cl}_{2}(70 \mathrm{~mL})$ and cooled to $-78{ }^{\circ} \mathrm{C}$. DIBAL-H (12 mL, 11.6 mmol, $1.0 \mathrm{M}$ in $\mathrm{CH}_{2} \mathrm{Cl}_{2}$ ) was added dropwise. The reaction was stirred at $-78{ }^{\circ} \mathrm{C}$ for $4 \mathrm{~h}$. The mixture was quenched with $\mathrm{MeOH}$ at $-78{ }^{\circ} \mathrm{C}$ and warmed to room temp. The mixture was stirred with aq sodium potassium tartrate solution until clear. The layers were separated and the organic layer was washed with $\mathrm{H}_{2} \mathrm{O}(40 \mathrm{~mL})$, dried over $\mathrm{Na}_{2} \mathrm{SO}_{4}$ and the solvent was removed. The residue was purified by flash column chromatography (1:20 EtOAc-hexane, $\mathrm{R}_{\mathrm{f}}$ $0.2)$ to give the title alcohol $(1.4 \mathrm{~g}, 73 \%)$ as a clear oil; $[\alpha]_{\mathrm{D}}+23\left(c 0.87, \mathrm{CHCl}_{3}\right) ;{ }^{1} \mathrm{H}$ NMR $\left(500 \mathrm{MHz}, \mathrm{CDCl}_{3}\right): \delta 5.88(\mathrm{ddd}, J=17.3,10.5,5.8 \mathrm{~Hz}, 1 \mathrm{H}, \mathrm{H}-7), 5.78-5.66(\mathrm{~m}, 2 \mathrm{H}, \mathrm{H}-2$, $\mathrm{H}-3$, overlapping signals), 5.17 (dt, $J=17.3,1.8 \mathrm{~Hz}, 1 \mathrm{H}, \mathrm{H}-8), 5.07$ (dt, $J=10.5,1.7 \mathrm{~Hz}, 1 \mathrm{H}$, H-8'), 4.30 (dd, $J=6.3,1.3 \mathrm{~Hz}, 1 \mathrm{H}, \mathrm{H}-4), 4.10$ (t, $J=5.8 \mathrm{~Hz}, 2 \mathrm{H}, \mathrm{H}-1, \mathrm{H}-1$ '), 4.05 (tt, $J=$ 6.0, $1.4 \mathrm{~Hz}, 1 \mathrm{H}, \mathrm{H}-6), 3.73$ (dd, $J=6.1,1.8 \mathrm{~Hz}, 1 \mathrm{H}, \mathrm{H}-5), 1.06-0.84$ (m, 27H, $\left.\mathrm{Si}\left(\mathrm{CH}_{2} \mathrm{CH}_{3}\right)_{3}\right), 0.71-0.42\left(\mathrm{~m}, 18 \mathrm{H},-\mathrm{Si}\left(\mathrm{CH}_{2} \mathrm{CH}_{3}\right)_{3}\right) ;{ }^{13} \mathrm{C} \mathrm{NMR}\left(126 \mathrm{MHz}, \mathrm{CDCl}_{3}\right) \delta 138.5$ (C-7), 132.2 (C-3), 130.5 (C-2), 115.0 (C-8), 80.4 (C-5), 75.2 (C-6), 72.6 (C-4), 63.6 (C-1), 6.9 - $6.9\left(-\mathrm{Si}\left(\mathrm{CH}_{2} \mathrm{CH}_{3}\right)_{3}\right), 5.2-4.3\left(-\mathrm{Si}\left(\mathrm{CH}_{2} \mathrm{CH}_{3}\right)_{3}\right)$; FTIR $3326(-\mathrm{OH}), 2954,2912,2877$, $1458,1414,1379,1238,1120,1083,1050,1003,972,921,835,788,719,672 \mathrm{~cm}^{-1}$; HRMS (ESI) $m / z$ calc for $\mathrm{C}_{26} \mathrm{H}_{55} \mathrm{O}_{4} \mathrm{Si}_{3}: 515.3408$, Found: 515.3430 [M-H]'.

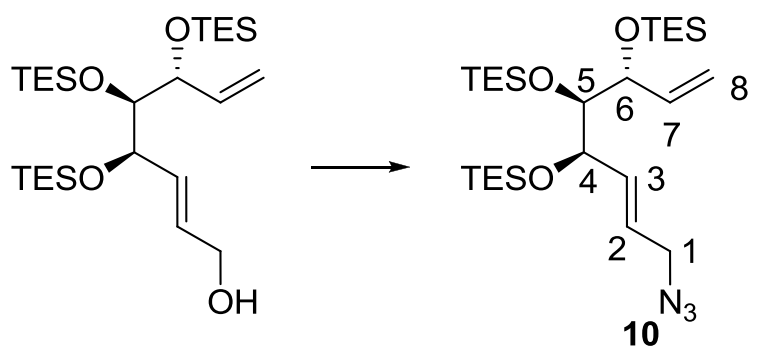

3,4,5-Tris(triethylsilyloxy) (3R,4S,5R,E)-8-azidoocta-1,6-diene 10 The precursor alcohol (1.4 g, $2.77 \mathrm{mmol})$ was dissolved in THF (40 mL). Triphenyl phosphine (1.24 g, 4.71 mmol) was added and the mixture was cooled to $0{ }^{\circ} \mathrm{C}$. Diisopropyl azodicarboxylate $(0.9 \mathrm{~mL}$, $4.71 \mathrm{mmol})$ and diphenylphosphoryl azide $(1.0 \mathrm{~mL}, 4.71 \mathrm{mmol})$ were added dropwise. The mixture was warmed to room temp and stirred for $8 \mathrm{~h}$. The solvent was evaporated and the 


\section{Supporting information - Experimental Section}

residue was purified by flash column chromatography (1:20 EtOAc-hexane, $\left.R_{f} 0.8\right)$ to give $10(1.2 \mathrm{~g}, 77 \%)$ as a pale yellow oil. $[\alpha]_{\mathrm{D}}+23.5\left(c 1.2, \mathrm{CHCl}_{3}\right) ;{ }^{1} \mathrm{H} \mathrm{NMR}\left(500 \mathrm{MHz}, \mathrm{CDCl}_{3}\right)$ $\delta 5.92-5.81$ (m, 2H, H-3, H-7, overlapping signals), 5.59 (dt, $J=15.5,6.6,1 \mathrm{H}, \mathrm{H}-2), 5.19$ (dt, $J=17.3,1.8 \mathrm{~Hz}, 1 \mathrm{H}, \mathrm{H}-8$ ), 5.09 (dt, $J=10.5,1.8 \mathrm{~Hz}, 1 \mathrm{H}, \mathrm{H}-8$ '), 4.33 (apt d, $J=6.6 \mathrm{~Hz}$, $1 \mathrm{H}, \mathrm{H}-4), 4.06$ (tt, $J=5.9,1.6 \mathrm{~Hz}, 1 \mathrm{H}, \mathrm{H}-6), 3.74$ (dd, $J=6.2,1.7 \mathrm{~Hz}, 1 \mathrm{H}, \mathrm{H}-5), 3.73-3.68$ (m, 2H, H-1, H-1', overlapping signals), $0.98-0.93\left(\mathrm{~m}, 27 \mathrm{H},-\mathrm{Si}\left(\mathrm{CH}_{2} \mathrm{CH}_{3}\right)_{3}\right), 0.65-0.56(\mathrm{~m}$, $\left.18 \mathrm{H},-\mathrm{Si}\left(\mathrm{CH}_{2} \mathrm{CH}_{3}\right)_{3}\right) ;{ }^{13} \mathrm{C}$ NMR (126 MHz, $\left.\mathrm{CDCl}_{3}\right) \delta 138.2(\mathrm{C}-7), 135.7$ (C-3), 124.5 (C-2), 115.1 (C-8), 80.4 (C-5), 75.2 (C-6), 72.3 (C-4), 52.7 (C-1), 6.9 - $6.8\left(-\mathrm{Si}_{(}\left(\mathrm{CH}_{2} \mathrm{CH}_{3}\right)_{3}\right), 5.0$ 4.9 (-Si( $\left.\left.\mathrm{CH}_{2} \mathrm{CH}_{3}\right)_{3}\right)$; FTIR 2955, 2912, 2877, $2097\left(-\mathrm{N}_{3}\right), 1458,1414,1379,1237,1119$, 1081, 1051, 1004, 971, 921, 884, 860, 834, 788, $725 \mathrm{~cm}^{-1}$; HRMS (ESI) $\mathrm{m} / z$ calc for $\mathrm{C}_{26} \mathrm{H}_{55} \mathrm{O}_{3} \mathrm{~N}_{3} \mathrm{Si}_{3} \mathrm{Na}$ : 564.3449, Found: 564.3439 [M+Na] ${ }^{+}$.
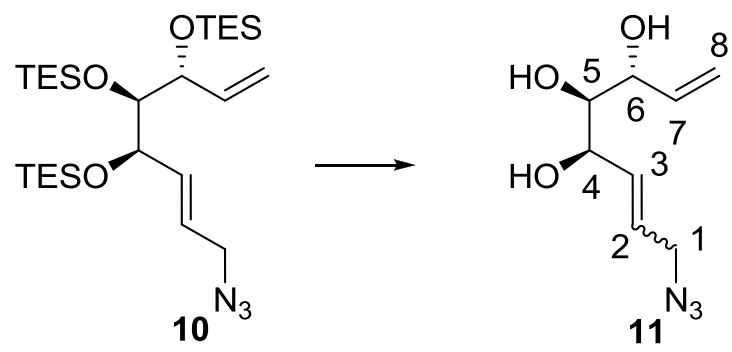

(3R,4S,5R)-8-Azidoocta-1,6-diene-3,4,5-triol 11 Compound 10 (1.1 g, 1.98 mmol) was dissolved in THF (30 mL). TBAF (12 mL, $11.86 \mathrm{mmol}, 1.0 \mathrm{M}$ in THF) was added slowly and the reaction was stirred at room temp for $12 \mathrm{~h}$. The solution was stirred with $\mathrm{CaCO}_{3}$, DOWEX-50X8 and $\mathrm{MeOH}$ for $1 \mathrm{~h}$. The slurry was filtered through a pad of celite and washed thoroughly with $\mathrm{MeOH}$ and the solvent was removed under diminished pressure. Flash column chromatography of the residue (1:1 EtOAc-hexane, $\left.\mathrm{R}_{\mathrm{f}} 0.15\right)$ gave $11(0.28 \mathrm{~g}$, $72 \%$ ), a mixture of isomers as a clear oil. Analytical data for major isomer: ${ }^{1} \mathrm{H}$ NMR (500 $\left.\mathrm{MHz}, \mathrm{CDCl}_{3}\right) \delta 5.95-5.87$ (m, 3H, H-2, H-3, H-7, overlapping signals), 5.38 (dt, $J=17.2$, $1.4 \mathrm{~Hz}, 1 \mathrm{H}, \mathrm{H}-8$ ), 5.28 (dt, $\left.J=10.5,1.4 \mathrm{~Hz}, 1 \mathrm{H}, \mathrm{H}-8^{\prime}\right), 4.37$ (t, $\left.J=4.3 \mathrm{~Hz}, 1 \mathrm{H}, \mathrm{H}-4\right), 4.34$ (ddt, $J=5.9,3.6,1.3 \mathrm{~Hz}, 1 \mathrm{H}, \mathrm{H}-6$ ), $3.88-3.81$ (m, 2H, H-1, H-1'), 3.56 (dd, $J=4.5,3.7 \mathrm{~Hz}$, 1H, H-5), 2.83 (s, -OH); ${ }^{13} \mathrm{C}$ NMR (126 MHz, $\left.\mathrm{CDCl}_{3}\right) \delta 136.9$ (C-7), 133.4 (C-3), 126.0 (C2), 117.4 (C-8), 75.0 (C-5), 73.5 (C-4), 72.4 (C-6), 52.1 (C-1); FTIR 3387 (-OH), 2919, 2100 $\left(-\mathrm{N}_{3}\right), 1749,1438,1371,1219,1037,980,930 \mathrm{~cm}^{-1}$; HRMS (ESI) $\mathrm{m} / z$ calc for $\mathrm{C}_{8} \mathrm{H}_{12} \mathrm{O}_{3} \mathrm{~N}_{3}$ : 198.0879, Found: 198.0886 [M-H]'. 


\section{Supporting information - Experimental Section}
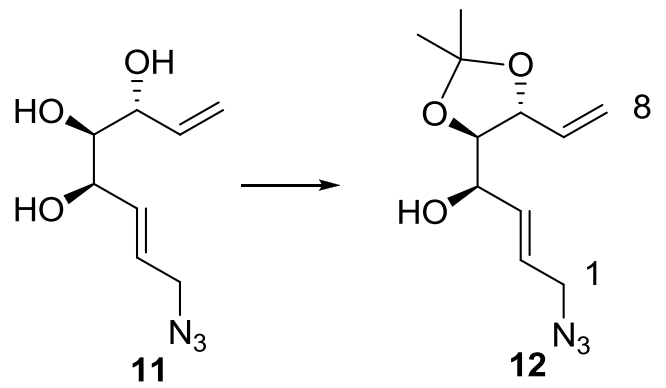

(R,E)-4-Azido-1-((4R,5R)-2,2-dimethyl-5-vinyl-1,3-dioxolan-4-yl)but-2-en-1-ol 12 Azide $11(0.7 \mathrm{~g}, 3.72 \mathrm{mmol})$ was dissolved in dry acetone $(50 \mathrm{~mL}$, dried over $4 \AA \hat{́}$ sieves $) . \mathrm{H}_{2} \mathrm{SO}_{4}$ (cat.) was added and the reaction stirred at room temp for $12 \mathrm{~h}$. The reaction was neutralised with aq $\mathrm{NaHCO}_{3}$ and evaporated to remove the acetone. The residue was re-dissolved in $\mathrm{CH}_{2} \mathrm{Cl}_{2}(40 \mathrm{~mL})$ and washed with $\mathrm{H}_{2} \mathrm{O}(20 \mathrm{~mL})$, dried over $\mathrm{Na}_{2} \mathrm{SO}_{4}$ and the solvent was removed to give $12(0.89 \mathrm{~g}, 78 \%)$ as a clear oil (1:1 EtOAc-hexane, $\left.\mathrm{R}_{\mathrm{f}} 0.8\right)$; $[\alpha]_{\mathrm{D}}-1.5(c$ 2.5, $\left.\mathrm{CHCl}_{3}\right) ;{ }^{1} \mathrm{H} \mathrm{NMR}\left(500 \mathrm{MHz}, \mathrm{CDCl}_{3}\right) \delta 5.87-5.67$ (m, 3H, H-2, H-3, H-7, overlapping signals), 5.33 (dt, $J=17.1,1.3 \mathrm{~Hz}, 1 \mathrm{H}, \mathrm{H}-8), 5.21$ (dt, $J=10.3,1.2 \mathrm{~Hz}, 1 \mathrm{H}, \mathrm{H}-8$ '), 4.39 4.35 (m, 2H, H-4, H-6), 3.79 - 3.74 (m, 3H, H-5, H-1, H-1', overlapping signals), 2.59 (s, $1 \mathrm{H},-\mathrm{OH}), 1.41\left(\mathrm{~s}, 3 \mathrm{H},-\mathrm{C}\left(\mathrm{CH}_{3}\right)_{2}\right), 1.39\left(\mathrm{~s}, 3 \mathrm{H},-\mathrm{C}\left(\mathrm{CH}_{3}\right)_{2}\right) ;{ }^{13} \mathrm{C} \mathrm{NMR}\left(126 \mathrm{MHz}, \mathrm{CDCl}_{3}\right) \delta$ 136.0 (C-7), 132.1, 125.7 (C-2, C-3), 118.7 (C-8), 109.3 (C, -C( $\left.\left.\mathrm{CH}_{3}\right)_{2}\right), 82.7$ (C-5), 77.7 (C6), 70.4 (C-4), 52.1 (t, C-1), $26.9\left(\mathrm{CH}_{3},-\mathrm{C}\left(\mathrm{CH}_{3}\right)_{2}\right), 26.9\left(\mathrm{CH}_{3},-\mathrm{C}\left(\mathrm{CH}_{3}\right)_{2}\right)$; FTIR 3460, 2988, 2937, 2887, $2099\left(-\mathrm{N}_{3}\right), 1645,1457,1373,1216,1168,1121,1055,976,910,873,814,727$ $\mathrm{cm}^{-1}$; HRMS (ESI) $\mathrm{m} / z$ calc for $\mathrm{C}_{11} \mathrm{H}_{19} \mathrm{~N}_{3} \mathrm{O}_{3}: 240.1348$, Found: $240.1349[\mathrm{M}+\mathrm{H}]^{+}$.

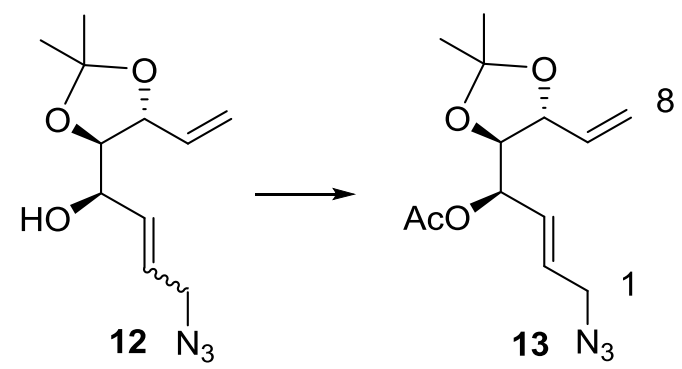

(R,E)-4-Azido-1-((4S,5R)-2,2-dimethyl-5-vinyl-1,3-dioxolan-4-yl)but-2-enyl acetate 13 Compound 12 (50 mg, $0.21 \mathrm{mmol})$ was dissolved in pyridine (5 mL, excess). $\mathrm{Ac}_{2} \mathrm{O}(20 \mu \mathrm{l}$, $0.23 \mathrm{mmol}$ ) and DMAP (2 mg, cat.) were added and stirred at room temp for $6 \mathrm{~h}$. The reaction was removed under diminished pressure. Flash column chromatography of the residue (1:4 EtOAc-hexane, $\left.\mathrm{R}_{\mathrm{f}} 0.5\right)$ gave $\mathbf{1 3}(47 \mathrm{mg}, 81 \%)$ as a pale yellow oil; $[\alpha]_{\mathrm{D}}-14(c$ $\left.0.8, \mathrm{CHCl}_{3}\right) ;{ }^{1} \mathrm{H} \mathrm{NMR}\left(500 \mathrm{MHz}, \mathrm{CDCl}_{3}\right) \delta 5.85-5.78$ (m, 3H, H-2, H-3, H-7, overlapping 


\section{Supporting information - Experimental Section}

signals), 5.42 (t, $J=5.0 \mathrm{~Hz}, 1 \mathrm{H}, \mathrm{H}-4), 5.36$ (apt d, $J=17.1 \mathrm{~Hz}, 1 \mathrm{H}, \mathrm{H}-8$ ), 5.27 (apt d, $J=$ $10.2 \mathrm{~Hz}, 1 \mathrm{H}, \mathrm{H}-8$ '), 4.25 (t, $J=7.8 \mathrm{~Hz}, 1 \mathrm{H}, \mathrm{H}-6$ ), 3.84 (dd, $J=8.3,4.6 \mathrm{~Hz}, 1 \mathrm{H}, \mathrm{H}-5$ ), 3.81 3.80 (m, 2H, H-1, H-1', overlapping signals), 2.07 (s, 3H, $\left.-\mathrm{OCH}_{3}\right), 1.42\left(\mathrm{~s}, 3 \mathrm{H},-\mathrm{C}\left(\mathrm{CH}_{3}\right)_{2}\right)$, $1.40\left(\mathrm{~s}, 3 \mathrm{H},-\mathrm{C}\left(\mathrm{CH}_{3}\right)_{2}\right) ;{ }^{13} \mathrm{C}$ NMR $\left(126 \mathrm{MHz} \mathrm{CDCl}_{3}\right) \delta 169.7(\mathrm{C}-1), 135.1(\mathrm{C}-7), 128.7,128.5$ (C-2, C-3), 119.3 (C-8), $109.8\left(\mathrm{C},-\mathrm{C}\left(\mathrm{CH}_{3}\right)_{2}\right), 81.1(\mathrm{C}-5), 79.4$ (C-6), 72.4 (C-4), 51.9 (C-1), $27.0\left(\mathrm{CH}_{3},-\mathrm{C}\left(\mathrm{CH}_{3}\right)_{2}\right), 26.7\left(\mathrm{CH}_{3},-\mathrm{C}\left(\mathrm{CH}_{3}\right)_{2}\right), 21.0\left(-\mathrm{OCH}_{3}\right)$; FTIR 2989, 2931, 2857, 2101 ($\left.\mathrm{N}_{3}\right), 1743,1713,1431,1372,1223,1172,1065,1021,984,934,872,811,715 \mathrm{~cm}^{-1}$; HRMS (ESI) $m / z$ calc for $\mathrm{C}_{26} \mathrm{H}_{39} \mathrm{O}_{8} \mathrm{~N}_{6}: 563.2829$, Found: $563.2857[2 \mathrm{M}+\mathrm{H}]^{+}$.

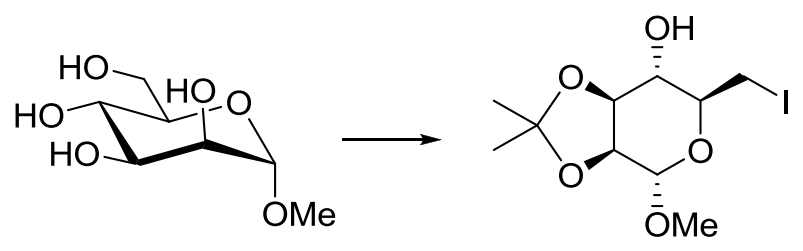

Methyl 6-deoxy-6-iodo-2,3- $O$-isopropylidene- $\alpha$-D-mannopyranoside Methyl $\alpha$-Dmannopyranoside (10.0 g, $0.051 \mathrm{~mol})$, imidazole (7.0 g, $0.103 \mathrm{~mol})$ and triphenyl phosphine (20.3 g, $0.077 \mathrm{~mol})$ were dissolved in THF $(150 \mathrm{~mL})$ and heated to $60{ }^{\circ} \mathrm{C}$. Iodine $(19.7 \mathrm{~g}$, $0.077 \mathrm{~mol}$ ) was added portion-wise and the reaction was heated at reflux for $2 \mathrm{~h}$. The mixture was cooled to room temp and the solvent was removed under diminished pressure. The residue was re-dissolved in a mixture of acetone $(300 \mathrm{~mL})$ and 2,2-DMP $(30 \mathrm{~mL})$ and cooled to $0{ }^{\circ} \mathrm{C}$. $p$ - $\mathrm{TsOH}(5.3 \mathrm{~g}, 0.031 \mathrm{~mol})$ was added and the mixture was stirred at room temp for 2 h. $\mathrm{Et}_{3} \mathrm{~N}(30 \mathrm{~mL})$ was added and the solvent was removed under diminished pressure. The crude residue was re-dissolved in $\mathrm{CH}_{2} \mathrm{Cl}_{2}(50 \mathrm{~mL})$, washed with $\mathrm{NaHCO}_{3}(40 \mathrm{~mL})$, dried over $\mathrm{Na}_{2} \mathrm{SO}_{4}$ and the solvent was removed. Flash column chromatography of the residue $(1: 4$ EtOAc-hexane) gave the title compound (12.6 g, $71 \%$ ) as a white solid. All analytical data corresponded well to literature data; ${ }^{2}$ TLC 1:4 EtOAc-hexane, $\left.\mathrm{R}_{\mathrm{f}} 0.35\right) ;[\alpha]_{\mathrm{D}}+33(c$ 1.1, $\mathrm{CHCl}_{3}$ ); ${ }^{1} \mathrm{H}$ NMR (500 MHz, $\mathrm{CDCl}_{3}$ ) $\delta 4.92$ (apt s, 1H, H-1), 4.16 - 4.09 (m, 2H, H-3, H-4, overlapping signals), 3.59 (dd, $J=10.6,2.7 \mathrm{~Hz}, 1 \mathrm{H}, \mathrm{H}-6), 3.54$ (m, 1H, H-2), 3.50 - 3.47 (m, $4 \mathrm{H}, \mathrm{H}-4,-\mathrm{OCH}_{3}$, overlapping signals), 3.31 (dd, $J=10.6,7.2 \mathrm{~Hz}, 1 \mathrm{H}, \mathrm{H}-6$ '), 1.52 (s, 3H, $\left.\mathrm{C}\left(\mathrm{CH}_{3}\right)_{2}\right), 1.35\left(\mathrm{~s}, 3 \mathrm{H},-\mathrm{C}\left(\mathrm{CH}_{3}\right)_{2}\right) ;{ }^{13} \mathrm{C} \mathrm{NMR}\left(126 \mathrm{MHz}, \mathrm{CDCl}_{3}\right) \delta 109.8\left(-\mathrm{C}\left(\mathrm{CH}_{3}\right)_{2}\right), 98.5(\mathrm{C}-$ 1), 78.2, 75.7 (C-3, C-4), $73.2(\mathrm{C}-2), 69.3(\mathrm{C}-5), 55.6\left(-\mathrm{OCH}_{3}\right), 28.0\left(-\mathrm{C}\left(\mathrm{CH}_{3}\right)_{2}\right), 26.1$ ($\left.\mathrm{C}\left(\mathrm{CH}_{3}\right)_{2}\right)$, 6.6 (C-6); FTIR 3442, 2985, 2937, 2910, 1456, 1444, 1402, 1383, 1308, 1269 , 1238, 1221, 1202, 1169, 1133, 1080, 1061, 1027, 988, 967, 956, 919, 848, 815, 781, $735 \mathrm{~cm}^{-}$ ${ }^{1}$; HRMS (ESI) $\mathrm{m} / \mathrm{z}$ calc for $\mathrm{C}_{10} \mathrm{H}_{18} \mathrm{O}_{5} \mathrm{I}$ : 345.0199, Found: $345.0188[\mathrm{M}+\mathrm{H}]^{+}$. 


\section{Supporting information - Experimental Section}

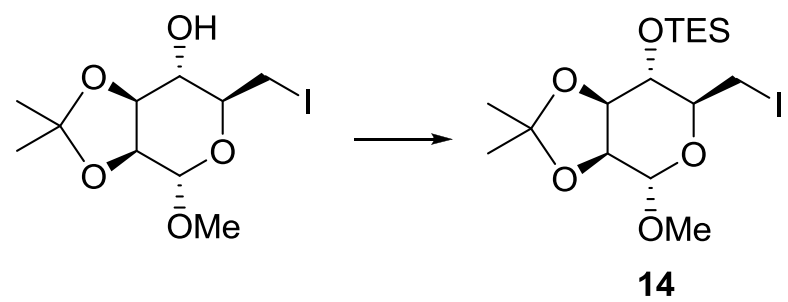

Compound 14. The precursor iodide (12.6 g, $3.66 \mathrm{~mol})$ was dissolved in $\mathrm{CH}_{2} \mathrm{Cl}_{2}(200 \mathrm{~mL})$. Imidazole $(4.99 \mathrm{~g}, 7.33 \mathrm{~mol})$ and chlorotriethylsilane $(15.4 \mathrm{~mL}, 7.33 \mathrm{~mol})$ were added and the mixture was stirred at room temp and water $(100 \mathrm{~mL})$ was charged. The layers were separated and the organic layer was dried over $\mathrm{Na}_{2} \mathrm{SO}_{4}$ and the solvent was removed under diminished pressure. Flash column chromatography of the residue (1:10 EtOAc-hexane) gave $14(11.8 \mathrm{~g}, 70 \%)$ as a clear oil. (TLC 1:4 EtOAc-hexane, $\left.\mathrm{R}_{\mathrm{f}} 0.9\right) ;[\alpha]_{\mathrm{D}}+36.8\left(c\right.$ 3.5, $\left.\mathrm{CHCl}_{3}\right)$; ${ }^{1} \mathrm{H}$ NMR (500 MHz, $\mathrm{CDCl}_{3}$ ) $\delta 4.91$ (apt s, 1H, H-1), 4.09 (dd, $\left.J=5.7,0.8 \mathrm{~Hz}, 1 \mathrm{H}, \mathrm{H}-2\right), 4.00$ (t, $J=6.0 \mathrm{~Hz}, 1 \mathrm{H}, \mathrm{H}-3), 3.56(\mathrm{dd}, J=10.4,2.0 \mathrm{~Hz}, 1 \mathrm{H}, \mathrm{H}-6), 3.48$ (s, 3H, $\left.-\mathrm{OCH}_{3}\right), 3.44-$ 3.41 (m, 2H, H-4, H-5, overlapping signals), 3.23 (dd, $J=10.4,7.3 \mathrm{~Hz}, 1 \mathrm{H}, \mathrm{H}-6$ '), 1.51 (s, $\left.3 \mathrm{H},-\mathrm{C}\left(\mathrm{CH}_{3}\right)_{2}\right), 1.33\left(\mathrm{~s}, 3 \mathrm{H},-\mathrm{C}\left(\mathrm{CH}_{3}\right)_{2}\right), 0.96\left(\mathrm{t}, J=7.9 \mathrm{~Hz}, 9 \mathrm{H},-\mathrm{Si}\left(\mathrm{CH}_{2} \mathrm{CH}_{3}\right)_{3}\right), 0.71-0.63$ $\left(\mathrm{m}, 6 \mathrm{H},-\mathrm{Si}\left(\mathrm{CH}_{2} \mathrm{CH}_{3}\right)_{3}\right) ;{ }^{13} \mathrm{C} \mathrm{NMR}\left(126 \mathrm{MHz}, \mathrm{CDCl}_{3}\right) \delta 109.3\left(-\mathrm{C}\left(\mathrm{CH}_{3}\right)_{2}\right), 98.4(\mathrm{C}-1), 78.9$ (C-3), 75.9 (C-2), 74.7 (C-4), $69.5(\mathrm{C}-5), 55.5\left(-\mathrm{OCH}_{3}\right), 28.0\left(-\mathrm{C}\left(\mathrm{CH}_{3}\right)_{2}\right), 26.3\left(-\mathrm{C}\left(\mathrm{CH}_{3}\right)_{2}\right)$, 7.20 (C-6), $6.8\left(\mathrm{CH}_{3},-\mathrm{Si}\left(\mathrm{CH}_{2} \mathrm{CH}_{3}\right)_{3}\right), 4.9\left(\mathrm{CH}_{2},-\mathrm{Si}\left(\mathrm{CH}_{2} \mathrm{CH}_{3}\right)_{3}\right)$; FTIR 2954, 2911, 2877 , 1742, 1458, 1414, 1381, 1372, 1243, 1219, 1168, 1122, 1090, 1026, 974, 960, 853, 776, 743, 727, $677 \mathrm{~cm}^{-1}$; HRMS (ESI) $\mathrm{m} / z$ calc for $\mathrm{C}_{16} \mathrm{H}_{35} \mathrm{O}_{5} \mathrm{ISiN}$ : 476.1329, Found: 476.1340 $\left[\mathrm{M}+\mathrm{NH}_{4}\right]^{+}$.

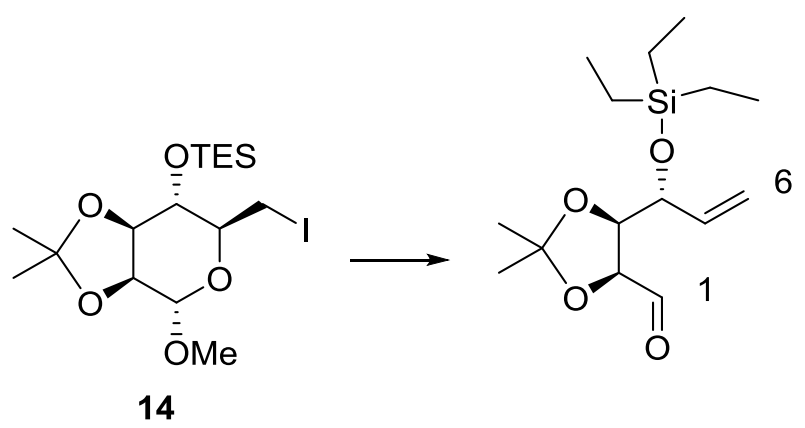

(4S,5R)-2,2-Dimethyl-5-((R)-1-(triethylsilyloxy)allyl)-1,3-dioxolane-4-carbaldehyde

Compound 14 (11.5 g, $0.025 \mathrm{~mol})$ was dissolved in a mixture of THF- $\mathrm{H}_{2} \mathrm{O}(9: 1,110 \mathrm{~mL})$. Pre-activated $\mathrm{Zn}$ dust $(16.4 \mathrm{~g}, 0.25 \mathrm{~mol})$ was added and the mixture was sonicated at $40{ }^{\circ} \mathrm{C}$ for $2 \mathrm{~h}$. The mixture was filtered and washed with $\mathrm{Et}_{2} \mathrm{O}(100 \mathrm{~mL})$. The organic layer was washed with $\mathrm{H}_{2} \mathrm{O}(80 \mathrm{~mL})$, satd aq $\mathrm{NaHCO}_{3}(80 \mathrm{~mL})$ and brine $(80 \mathrm{~mL})$, dried over $\mathrm{Na}_{2} \mathrm{SO}_{4}$ and the solvent was removed to give the title compound $(5.95 \mathrm{~g}, 79 \%)$ as a clear oil; $[\alpha]_{\mathrm{D}}$ - 


\section{Supporting information - Experimental Section}

$3.1\left(\right.$ c 10, $\left.\mathrm{CHCl}_{3}\right) ;{ }^{1} \mathrm{H}$ NMR $\left(500 \mathrm{MHz}, \mathrm{CDCl}_{3}\right) \delta 9.69(\mathrm{t}, J=1.7 \mathrm{~Hz}, 1 \mathrm{H}, \mathrm{H}-1), 5.95$ (ddd, $J=$ 7.4, 10.3, $17.8 \mathrm{~Hz}, 1 \mathrm{H}, \mathrm{H}-5), 5.26$ (dd, $J=17.4,1.3 \mathrm{~Hz}, 1 \mathrm{H}, \mathrm{H}-6), 5.18$ (dq, $J=10.3,0.7 \mathrm{~Hz}$, 1H, H-6'), 4.38 (dt, $J=7.8,1.7$ Hz, 1H, H-2), 4.34 (ddd, $J=7.8,3.4,1.4 \mathrm{~Hz}, 1 \mathrm{H}, \mathrm{H}-3$ ), 4.24 -2.21 (m, 1H, H-4), 1.56 (s, 3H, - $\left.\mathrm{C}\left(\mathrm{CH}_{3}\right)_{2}\right), 1.35$ (s, 3H, - $\left.\mathrm{C}\left(\mathrm{CH}_{3}\right)_{2}\right), 0.92$ (t, J = 8.0 Hz, 9H, $\left.\mathrm{Si}\left(\mathrm{CH}_{2} \mathrm{CH}_{3}\right)_{3}\right), 0.56$ (q, $\left.J=7.9 \mathrm{~Hz}, 6 \mathrm{H},-\mathrm{Si}\left(\mathrm{CH}_{2} \mathrm{CH}_{3}\right)_{3}\right) ;{ }^{13} \mathrm{C} \mathrm{NMR}\left(126 \mathrm{MHz}, \mathrm{CDCl}_{3}\right) \delta 200.5$ (C-1), 137.1 (C-5), 117.5 (C-6), 110.8 (-C(CH$\left.)_{2}\right), 83.5$ (C-3), 81.1 (C-2), 72.5 (C-4), 26.6 ($\left.\mathrm{C}\left(\mathrm{CH}_{3}\right)_{2}\right), 25.2\left(-\mathrm{C}\left(\mathrm{CH}_{3}\right)_{2}\right), 6.7\left(\mathrm{CH}_{3},-\mathrm{Si}\left(\mathrm{CH}_{2} \mathrm{CH}_{3}\right)_{3}\right), 5.1\left(\mathrm{CH}_{2},-\mathrm{Si}\left(\mathrm{CH}_{2} \mathrm{CH}_{3}\right)_{3}\right)$; FTIR 2955, 2913, 2878, 1732, 1458, 1416, 1381, 1372, 1241, 1215, 1126, 1066, 1003, 928, 831, 727, 972 $\mathrm{cm}^{-1}$; HRMS (ESI) $\mathrm{m} / z$ calc for $\mathrm{C}_{15} \mathrm{H}_{28} \mathrm{O}_{4} \mathrm{SiNa}$ : 323.1655, Found: $323.1664[\mathrm{M}+\mathrm{Na}]^{+}$.

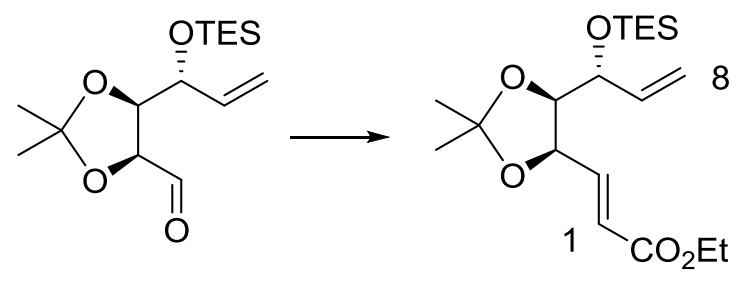

15

(E)-Ethyl 3-((4R,5R)-2,2-dimethyl-5-((R)-1-(triethylsilyloxy)allyl)-1,3-dioxolan-4yl)acrylate 15 The precursor aldehyde $(6.0 \mathrm{~g}, 0.02 \mathrm{~mol})$ was dissolved in toluene (100 $\mathrm{mL})$. (Carbethoxy methylene) triphenylphosphorane $(10.4 \mathrm{~g}, 0.03 \mathrm{~mol})$ was added and the reaction was heated to reflux for $12 \mathrm{~h}$. The solvent was removed to a yellow solid. The residue was purified by column chromatography (1:10 EtOAc-hexane) to give $\mathbf{1 5}(6.1 \mathrm{~g}, 83$ $\%)$ as a clear oil. (TLC 1:9 EtOAc-hexane, $\left.\mathrm{R}_{\mathrm{f}} 0.7\right)$; $[\alpha]_{\mathrm{D}}+15.3\left(c 4.9, \mathrm{CHCl}_{3}\right) ;{ }^{1} \mathrm{H}$ NMR (500 $\left.\mathrm{MHz}, \mathrm{CDCl}_{3}\right) \delta 7.02(\mathrm{dd}, J=15.7,6.2 \mathrm{~Hz}, 1 \mathrm{H}, \mathrm{H}-3), 6.02(\mathrm{dd}, J=15.7,1.5 \mathrm{~Hz}, 1 \mathrm{H}, \mathrm{H}-2)$, 5.84 (dddd, $J=16.8,10.4,4.1,2.1 \mathrm{~Hz}, 1 \mathrm{H}, \mathrm{H}-7$ ), 5.28 (d, $J=17.2 \mathrm{~Hz}, 1 \mathrm{H}, \mathrm{H}-8$ '), 5.17 (dd, $J$ $\left.=10.4,1.7 \mathrm{~Hz}, 1 \mathrm{H}, \mathrm{H}-8^{\prime}\right), 4.65$ (td, $\left.J=6.4,1.5 \mathrm{~Hz}, 1 \mathrm{H}, \mathrm{H}-4\right), 4.20$ (q, $J=7.1 \mathrm{~Hz}, 2 \mathrm{H}$, $\mathrm{CO}_{2} \mathrm{Et}$ ), $4.16-4.13$ (m, 2H, H-5, H-6, overlapping signals), 1.51 (s, 3H, $\left.-\mathrm{C}\left(\mathrm{CH}_{3}\right)_{2}\right), 1.36$ (s, $\left.3 \mathrm{H},-\mathrm{C}\left(\mathrm{CH}_{3}\right)_{2}\right), 1.28\left(\mathrm{t}, J=7.1 \mathrm{~Hz}, 3 \mathrm{H},-\mathrm{CO}_{2} \mathrm{Et}\right), 0.94\left(\mathrm{t}, J=7.9 \mathrm{~Hz}, 9 \mathrm{H},-\mathrm{Si}\left(\mathrm{CH}_{2} \mathrm{CH}_{3}\right)_{3}\right), 0.59$ $\left(\mathrm{qd}, J=7.9,1.2 \mathrm{~Hz}, 6 \mathrm{H},-\mathrm{Si}\left(\mathrm{CH}_{2} \mathrm{CH}_{3}\right)_{3}\right) ;{ }^{13} \mathrm{C} \mathrm{NMR}\left(126 \mathrm{MHz}, \mathrm{CDCl}_{3}\right) \delta 165.9(\mathrm{C}-1), 143.7$ (C-3), 136.7 (C-7), 122.8 (C-2), 117.4 (C-8), $109.3\left(-\mathrm{C}\left(\mathrm{CH}_{3}\right)_{2}\right), 81.6$ (C-5), 76.5 (C-4), 72.9 (C-6), $60.4\left(-\mathrm{CO}_{2} \mathrm{Et}\right), 27.3 \quad\left(-\mathrm{C}\left(\mathrm{CH}_{3}\right)_{2}\right), 25.3 \quad\left(-\mathrm{C}\left(\mathrm{CH}_{3}\right)_{2}\right), 14.2 \quad\left(-\mathrm{CO}_{2} \mathrm{Et}\right), 6.7\left(\mathrm{CH}_{3}\right.$, $\left.\mathrm{Si}\left(\mathrm{CH}_{2} \mathrm{CH}_{3}\right)_{3}\right), 4.9\left(\mathrm{CH}_{2},-\mathrm{Si}\left(\mathrm{CH}_{2} \mathrm{CH}_{3}\right)_{3}\right)$; FTIR 2956, 2909, 2877, 1722, 1659, 1459, 1416, 1369, 1303, 1250, 1216, 1159, 1063, 1034, 1005, 979, 931, 884, 837, 802, $727 \mathrm{~cm}^{-1}$; HRMS (ESI) $m / z$ calc for $\mathrm{C}_{19} \mathrm{H}_{34} \mathrm{O}_{5}$ SiNa: 393.2073, Found: $393.2060[\mathrm{M}+\mathrm{Na}]^{+}$. 


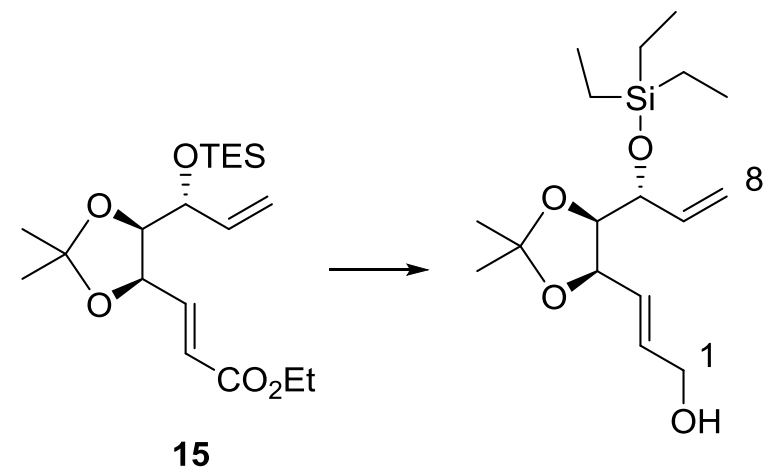

(E)-3-((4R,5R)-2,2-dimethyl-5-((R)-1-(triethylsilyloxy)allyl)-1,3-dioxolan-4-yl)prop-2-en-

1-ol Compound 15 (6.0 g, $0.016 \mathrm{~mol})$ was dissolved in $\mathrm{CH}_{2} \mathrm{Cl}_{2}(80 \mathrm{~mL})$ and cooled to -78 ${ }^{\circ} \mathrm{C}$. DIBAL-H (49 mL, $0.049 \mathrm{~mol}, 1.0 \mathrm{M}$ in $\mathrm{CH}_{2} \mathrm{Cl}_{2}$ ) was added dropwise. The reaction was stirred at $-78{ }^{\circ} \mathrm{C}$ for $4 \mathrm{~h}$. The mixture was quenched with $\mathrm{MeOH}$ at $-78{ }^{\circ} \mathrm{C}$ and warmed to room temp. The mixture was stirred with aq sodium potassium tartrate solution until clear. The layers were separated and the organic layer was washed with $\mathrm{H}_{2} \mathrm{O}(40 \mathrm{~mL})$, dried over $\mathrm{Na}_{2} \mathrm{SO}_{4}$ and the solvent was removed to give the title compound $(4.41 \mathrm{~g}, 84 \%)$ as a clear oil. (1:9 EtOAc-hexane, $\mathrm{R}_{\mathrm{f}}$ 0.2); $[\alpha]_{\mathrm{D}}+20.6\left(c 1.0\right.$ in $\left.\mathrm{CHCl}_{3}\right) ;{ }^{1} \mathrm{H} \mathrm{NMR}\left(500 \mathrm{MHz}, \mathrm{CDCl}_{3}\right) \delta 5.89$ - 5.82 (m, 3H, H-2, H-3, H-7, overlapping siganls), 5.30 (dt, $J=17.2,1.7 \mathrm{~Hz}, 1 \mathrm{H}, \mathrm{H}-8$ ), 5.14 (dt, $J=10.5,1.6 \mathrm{~Hz}, 1 \mathrm{H}, \mathrm{H}-8^{\prime}$ ), 4.50 (t, $\left.J=6.8 \mathrm{~Hz}, 1 \mathrm{H}, \mathrm{H}-4\right), 4.16-4.14$ (m, 3H, H-1, H-1', H-6, overlapping signals), 3.98 (t, $J=6.5 \mathrm{~Hz}, 1 \mathrm{H}, \mathrm{H}-5), 1.48$ (s, 3H, -C(CH3) $\left.)_{2}\right), 1.34$ (s, 3H, $\left.\mathrm{C}\left(\mathrm{CH}_{3}\right)_{2}\right), 0.94\left(\mathrm{t}, J=7.9 \mathrm{~Hz}, 9 \mathrm{H},-\mathrm{Si}\left(\mathrm{CH}_{2} \mathrm{CH}_{3}\right)_{3}\right), 0.60(\mathrm{qd}, J=7.9,2.8 \mathrm{~Hz}, 6 \mathrm{H}$, $\left.\mathrm{Si}\left(\mathrm{CH}_{2} \mathrm{CH}_{3}\right)_{3}\right) ;{ }^{13} \mathrm{C}$ NMR (126 MHz, $\left.\mathrm{CDCl}_{3}\right) \delta 137.0$ (C-7), 133.7 (C-2), 127.3 (C-3), 116.6 (C-8), $108.6\left(-\mathrm{C}\left(\mathrm{CH}_{3}\right)_{2}, 81.7\right.$ (C-5), 78.1 (C-4), 72.6 (C-6), $\left.62.8(\mathrm{C}-1), 27.7\left(-\mathrm{C}_{(\mathrm{CH}}\right)_{2}\right), 25.5$ $\left(-\mathrm{C}\left(\mathrm{CH}_{3}\right)_{2}\right), 6.8\left(\mathrm{CH}_{3},-\mathrm{Si}\left(\mathrm{CH}_{2} \mathrm{CH}_{3}\right)_{3}\right), 5.0\left(\mathrm{CH}_{2},-\mathrm{Si}\left(\mathrm{CH}_{2} \mathrm{CH}_{3}\right)_{3}\right)$; FTIR 3423, 2954, 2913, 2877, 1742, 1458, 1415, 1370, 1239, 1218, 1145, 1126, 1093, 1059, 1003, 974, 928, 868, $845,741,725 \mathrm{~cm}^{-1}$; HRMS (ESI) $\mathrm{m} / z$ calc for $\mathrm{C}_{34} \mathrm{H}_{63} \mathrm{O}_{8} \mathrm{Se}: 655.4062$, Found: 655.4061 [2M$\mathrm{H}]^{-}$.

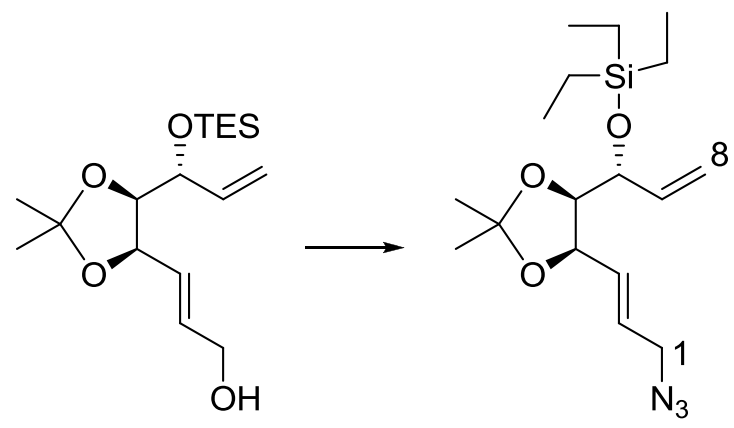




\section{Supporting information - Experimental Section}

((R)-1-((4R,5R)-5-((E)-3-azidoprop-1-enyl)-2,2-dimethyl-1,3-dioxolan-4-

yl)allyloxy)triethylsilane The alcohol precursor $(4.4 \mathrm{~g}, 0.013 \mathrm{~mol})$ was dissolved in THF $(80 \mathrm{~mL})$. Triphenyl phosphine $(6.0 \mathrm{~g}, 0.023 \mathrm{~mol})$ was added and the mixture was cooled to 0 ${ }^{\circ} \mathrm{C}$. Diisopropyl azodicarboxylate $(4.5 \mathrm{~mL}, 0.023 \mathrm{~mol})$ and diphenylphosphoryl azide (4.9 $\mathrm{mL}, 0.023 \mathrm{~mol}$ ) were added dropwise. The mixture was warmed to room temp and stirred for $8 \mathrm{~h}$. The solvent was removed under diminished pressure. Flash column chromatography of the residue (1:6 EtOAc-hexane) gave the title compound (3.46 g, $73 \%$ ) as a clear oil; (TLC 1:9 EtOAc-hexane, $\left.\mathrm{R}_{\mathrm{f}} 0.7\right) ;[\alpha]_{\mathrm{D}}+18\left(c\right.$ 1.5, $\left.\mathrm{CHCl}_{3}\right) ;{ }^{1} \mathrm{H} \mathrm{NMR}\left(500 \mathrm{MHz}, \mathrm{CDCl}_{3}\right) \delta 5.94-$ 5.84 (m, 2H, H-3, H-7, overlapping signals), 5.77 (dt, $J=15.4,6.1 \mathrm{~Hz}, 1 \mathrm{H}, \mathrm{H}-2), 5.32$ (d, $J=$ $18.3 \mathrm{~Hz}, 1 \mathrm{H}, \mathrm{H}-1), 5.17$ (d, $J=10.4 \mathrm{~Hz}, 1 \mathrm{H}, \mathrm{H}-1$ '), 4.53 (t, $J=7.0 \mathrm{~Hz}, 1 \mathrm{H}, \mathrm{H}-4), 4.17$ (t, $J=$ $6.4 \mathrm{~Hz}, 1 \mathrm{H}, \mathrm{H}-6), 4.02$ (t, $J=6.5 \mathrm{~Hz}, 1 \mathrm{H}, \mathrm{H}-5$ ), 3.80 (apt d, $J=6.0 \mathrm{~Hz}, 2 \mathrm{H}, \mathrm{H}-1, \mathrm{H}-1$ ') 1.50 $\left(\mathrm{s}, 3 \mathrm{H},-\mathrm{C}\left(\mathrm{CH}_{3}\right)_{2}\right), 1.36\left(\mathrm{~s}, 3 \mathrm{H},-\mathrm{C}\left(\mathrm{CH}_{3}\right)_{2}\right), 0.96\left(\mathrm{t}, J=7.9 \mathrm{~Hz}, 9 \mathrm{H},-\mathrm{Si}\left(\mathrm{CH}_{2} \mathrm{CH}_{3}\right)_{3}\right), 0.62(\mathrm{qd}, J$ $\left.=7.9,2.1 \mathrm{~Hz}, 6 \mathrm{H},-\mathrm{Si}\left(\mathrm{CH}_{2} \mathrm{CH}_{3}\right)_{3}\right) ;{ }^{13} \mathrm{C} \mathrm{NMR}\left(126 \mathrm{MHz}, \mathrm{CDCl}_{3}\right) \delta 136.8(\mathrm{C}-7), 131.2(\mathrm{C}-3)$, 127.2 (C-2), 116.9 (C-8), $108.8\left(-\mathrm{C}\left(\mathrm{CH}_{3}\right)_{2}\right), 81.6$ (C-5), 77.6 (C-4), 72.6 (C-6), 52.1 (C-1), $27.7\left(-\mathrm{C}\left(\mathrm{CH}_{3}\right)_{2}\right), 25.5\left(-\mathrm{C}\left(\mathrm{CH}_{3}\right)_{2}\right), 6.8\left(\mathrm{CH}_{3},-\mathrm{Si}\left(\mathrm{CH}_{2} \mathrm{CH}_{3}\right)_{3}\right), 5.0\left(\mathrm{CH}_{2},-\mathrm{Si}\left(\mathrm{CH}_{2} \mathrm{CH}_{3}\right)_{3}\right)$; FTIR 2935, 2913, 2877, $2099\left(-\mathrm{N}_{3}\right), 1741,1458,1415,1380,1370,1240,1218,1144,1125,1092$, 1060, 1006, 974, 930, 866, 844, 915, 777, 741, $725 \mathrm{~cm}^{-1}$; HRMS (ESI) $\mathrm{m} / z$ calc for $\mathrm{C}_{17} \mathrm{H}_{32} \mathrm{O}_{3} \mathrm{~N}_{3} \mathrm{Si}:$ 354.2213, Found: $354.2201[\mathrm{M}+\mathrm{H}]^{+}$.

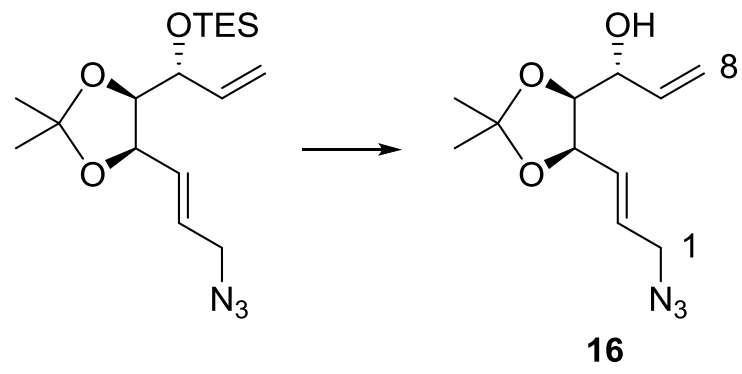

(R)-1-((4S,5R)-5-((E)-3-Azidoprop-1-enyl)-2,2-dimethyl-1,3-dioxolan-4-yl)prop-2-en-1-

ol 16 The silylated precursor $(3.5 \mathrm{~g}, 9.77 \mathrm{mmol})$ was dissolved in THF (80 mL). TBAF (29 $\mathrm{mL}, 0.029 \mathrm{~mol}, 1.0 \mathrm{M}$ in THF) was added slowly and the reaction was stirred at room temp for $12 \mathrm{~h}$. The solution was stirred with $\mathrm{CaCO}_{3}$, DOWEX-50X8 and $\mathrm{MeOH}$ for $1 \mathrm{~h}$. The slurry was filtered through a pad of celite and washed thoroughly with $\mathrm{MeOH}$ and the solvent was removed under diminished pressure. Flash column chromatography of the residue $(1: 1$ EtOAc-hexane) gave 16 (1.7 g, $73 \%$ ), which was a mixture of isomers, as a clear oil (TLC, 1:4 EtOAc-hexane $\mathrm{R}_{\mathrm{f}} 0.1$ ); ${ }^{1} \mathrm{H}$ NMR (500 MHz, $\left.\mathrm{CDCl}_{3}\right) \delta 5.97$ (ddt, $J=15.4,7.7,1.3 \mathrm{~Hz}$, $1 \mathrm{H}, \mathrm{H}-3$ ), $5.87-5.81$ (m, 2H, H-2, H-7, overlapping signals), 5.37 (apt d, $J=17.2 \mathrm{~Hz}, 1 \mathrm{H}$, H-8), 5.25 (apt d, $J=10.5 \mathrm{~Hz}, 1 \mathrm{H}, \mathrm{H}-8$ '), 4.66 (apt t, $J=6.8 \mathrm{~Hz}, 1 \mathrm{H}, \mathrm{H}-4$ ) , $4.14-4.10$ (m, 


\section{Supporting information - Experimental Section}

2H, H-5, H-6, overlapping signals), 3.83 - 3.82 (m, 2H, H-1, H-1', overlapping signals), 1.54 (s, 3H, - $\left.\mathrm{C}\left(\mathrm{CH}_{3}\right)_{2}\right), 1.40\left(\mathrm{~s}, 3 \mathrm{H},-\mathrm{C}\left(\mathrm{CH}_{3}\right)_{2}\right) ;{ }^{13} \mathrm{C} \mathrm{NMR}\left(126 \mathrm{MHz}, \mathrm{CDCl}_{3}\right) \delta 136.7(\mathrm{C}-7), 130.7$ (C-3), 128.2 (C-2), 117.4 (C-8), $109.0\left(-\mathrm{C}\left(\mathrm{CH}_{3}\right)_{2}\right), 80.5$ (C-5), 77.6 (C-4), 70.7 (C-6), 52.0 (C-1), $27.3\left(-\mathrm{C}\left(\mathrm{CH}_{3}\right)_{2}\right), 24.9\left(-\mathrm{C}\left(\mathrm{CH}_{3}\right)_{2}\right)$; FTIR 3449, 2988, 2935, 2909, $2099\left(-\mathrm{N}_{3}\right), 1738$, 1643, 1455, 1372, 1218, 1165, 1139, 1085, 1048, 974, 931, 859, $789 \mathrm{~cm}^{-1}$; HRMS (ESI) $\mathrm{m} / z$ calc for $\mathrm{C}_{22} \mathrm{H}_{34} \mathrm{O}_{6} \mathrm{~N}_{6} \mathrm{Na}$ : 501.2438, Found: $501.2421[2 \mathrm{M}+\mathrm{Na}]^{+}$.
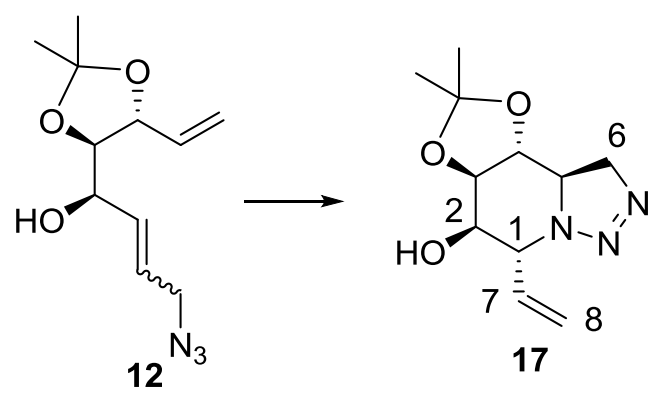

Triazoline 17 Azide 12 (150 mg, $0.63 \mathrm{mmol}$ ) was dissolved in DMF (30 mL) and heated to $100{ }^{\circ} \mathrm{C}$ for $6 \mathrm{~h}$. The mixture cooled to room temp and diluted with $\mathrm{H}_{2} \mathrm{O}(30 \mathrm{~mL})$. The aq layer was extracted with $\mathrm{Et}_{2} \mathrm{O}$ until no product remained in the aq layer (as observed by TLC, 1:1 EtOAc-hexane, $\mathrm{R}_{\mathrm{f}} 0.5$, visualised by $\mathrm{UV}$ and staining). The combined organic layers were dried over $\mathrm{Na}_{2} \mathrm{SO}_{3}$ and the solvent was removed. Flash column chromatography (1:4 to 1:2 EtOAc-hexane) gave $17(90 \mathrm{mg}, 60 \%)$ as a clear oil; $[\alpha]_{\mathrm{D}}-65\left(c 1.8, \mathrm{CHCl}_{3}\right) ;{ }^{1} \mathrm{H} \mathrm{NMR}(500$ $\mathrm{MHz}, \mathrm{CDCl}_{3}$ ) $\delta 5.84(\mathrm{ddd}, J=17.2,10.6,3.7 \mathrm{~Hz}, 1 \mathrm{H}, \mathrm{H}-7$ ), 5.35 (apt dd, $J=17.2,1.5 \mathrm{~Hz}$, 1H, H-8), 5.30 (apt dd, $J=10.6,1.6 \mathrm{~Hz}, 1 \mathrm{H}, \mathrm{H}-8$ '), 5.25 (dq, $J=4.1,2.2 \mathrm{~Hz}, 1 \mathrm{H}, \mathrm{H}-1$ ), 4.54 (dd, $J=16.1,2.7 \mathrm{~Hz}, 1 \mathrm{H}, \mathrm{H}-6), 4.42$ (apt s, 1H, H-2), 4.01 (dd, $J=16.1,10.0 \mathrm{~Hz}, 1 \mathrm{H}, \mathrm{H}-6$ '), $3.75(\mathrm{td}, J=9.7,2.7 \mathrm{~Hz}, 1 \mathrm{H}, \mathrm{H}-5), 3.53-3.47$ (m, 2H, H-3, H-4, overlapping signals), 2.84 (s, $1 \mathrm{H},-\mathrm{OH}), 1.39\left(\mathrm{~s}, 3 \mathrm{H},-\mathrm{C}\left(\mathrm{CH}_{3}\right)_{2}\right), 1.37\left(\mathrm{~s}, 3 \mathrm{H},-\mathrm{C}\left(\mathrm{CH}_{3}\right)_{2}\right) ;{ }^{13} \mathrm{C} \mathrm{NMR}\left(126 \mathrm{MHz}, \mathrm{CDCl}_{3}\right) \delta$ 133.7 (C-7), 117.4 (C-8), 110.4 (C, -C( $\left.\left.\mathrm{CH}_{3}\right)_{2}\right), 77.1$ (C-3), 70.2 (C-2), 69.1 (C-4), 67.6 (C-6), 63.3 (C-1), 56.3 (C-5), $26.8\left(\mathrm{CH}_{3},-\mathrm{C}\left(\mathrm{CH}_{3}\right)_{2}\right), 26.6\left(\mathrm{CH}_{3},-\mathrm{C}\left(\mathrm{CH}_{3}\right)_{2}\right)$; FTIR 3402, 2986, 2934, 1592, 1560, 1491, 1372, 1228, 1141, 1084, 1062, 1034, 924, 834, 778, 730, 715, $662 \mathrm{~cm}^{-1}$; HRMS (ESI) $m / z$ calc for $\mathrm{C}_{11} \mathrm{H}_{18} \mathrm{O}_{3} \mathrm{~N}_{3}: 240.1348$, Found: $240.1344[\mathrm{M}+\mathrm{H}]^{+}$. 


\section{Supporting information - Experimental Section}

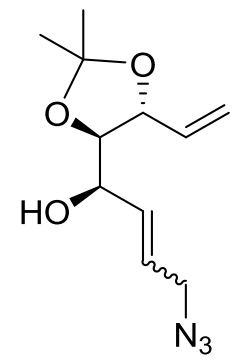

12

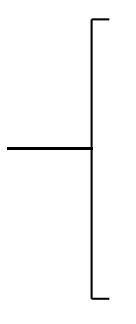

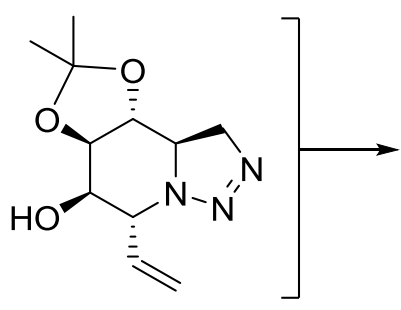

17

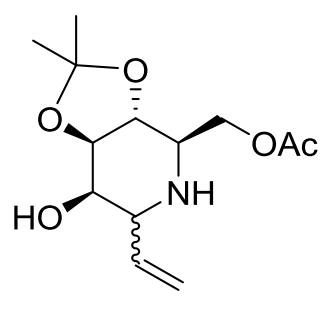

18

Piperidine 18 Azide 12 (220 mg, $0.92 \mathrm{mmol})$ was dissolved in toluene (20 mL) and heated to reflux for $6 \mathrm{~h}$. The reaction was cooled to $50{ }^{\circ} \mathrm{C}$. Acetic acid $(0.3 \mathrm{~mL}, 4.6 \mathrm{mmol})$ was added slowly, evolution of gas was observed along with a colour change from yellow to orange. After $5 \mathrm{~min}$ the reaction was judged to be complete by TLC and the solvent was removed. Flash column chromatography of the residue (2:1 EtOAc-hexane) gave $\mathbf{1 8}$ as a yellow oil $(8.7 \mathrm{mg}, 35 \%)$ and mixture of anomers; ${ }^{1} \mathrm{H}$ NMR of major anomer $(500 \mathrm{MHz}$, $\left.\mathrm{CDCl}_{3}\right) \delta 5.79(\mathrm{ddd}, J=17.4,10.7,4.1 \mathrm{~Hz}, 1 \mathrm{H}, \mathrm{H}-7), 5.36(\mathrm{ddd}, J=17.4,2.3,1.3 \mathrm{~Hz}, 1 \mathrm{H}, \mathrm{H}-$ 8'), 5.24 (ddd, $J=10.7,2.5,1.3 \mathrm{~Hz}, 1 \mathrm{H}, \mathrm{H}-8$ '), 4.34 - 4.28 (m, 2H, H-2, H-6, overlapping signals), 4.15 (dd, $J=11.5,5.7 \mathrm{~Hz}, 1 \mathrm{H}, \mathrm{H}-6$ ') 3.78 (dq, $J=4.4,2.3 \mathrm{~Hz}, 1 \mathrm{H}, \mathrm{H}-1), 3.70$ (t, $J=$ $9.7 \mathrm{~Hz}, 1 \mathrm{H}, \mathrm{H}-4), 3.49$ (dd, $J=9.3,2.3 \mathrm{~Hz}, 1 \mathrm{H}, \mathrm{H}-3$ ), 3.20 (ddd, $J=10.2,5.8,3.2 \mathrm{~Hz}, 1 \mathrm{H}, \mathrm{H}-$ 5), $2.09\left(\mathrm{~s}, 3 \mathrm{H},-\mathrm{COOCH}_{3}\right), 1.42\left(\mathrm{~s}, 3 \mathrm{H},-\mathrm{C}\left(\mathrm{CH}_{3}\right)_{2}\right), 1.40\left(\mathrm{~s}, 3 \mathrm{H},-\mathrm{C}\left(\mathrm{CH}_{3}\right)_{2}\right) ;{ }^{13} \mathrm{C} \mathrm{NMR}(126$ $\left.\mathrm{MHz}, \mathrm{CDCl}_{3}\right) \delta 170.8(\mathrm{C}=\mathrm{O}), 135.3(\mathrm{C}-7), 116.8(\mathrm{C}-8), 109.3\left(\mathrm{C},-\mathrm{C}\left(\mathrm{CH}_{3}\right)_{2}\right), 78.3(\mathrm{C}-3), 71.8$ (C-4), 70.9 (C-2), 64.6 (C-6), 59.8 (C-1), $53.4(\mathrm{C}-5), 26.8,26.6\left(\mathrm{CH}_{3},-\mathrm{C}\left(\mathrm{CH}_{3}\right)_{2}\right), 20.8\left(\mathrm{CH}_{3}\right.$, $\left.\mathrm{C}\left(\mathrm{CH}_{3}\right)_{2}\right)$; HRMS (ESI) $\mathrm{m} / z$ calc for $\mathrm{C}_{13} \mathrm{H}_{22} \mathrm{O}_{5} \mathrm{~N}$ : 272.1498, Found: $272.1504[\mathrm{M}+\mathrm{H}]^{+} ; \mathrm{HRMS}$ (ESI) $m / z$ calc for $\mathrm{C}_{13} \mathrm{H}_{20} \mathrm{O}_{5} \mathrm{~N}: 270.1341$ Found: $270.1340[\mathrm{M}-\mathrm{H}]^{-}$.

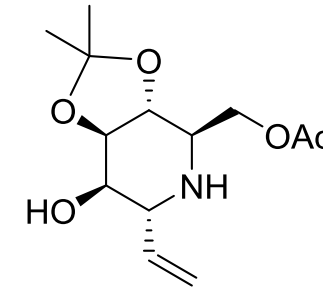

$18 \alpha$

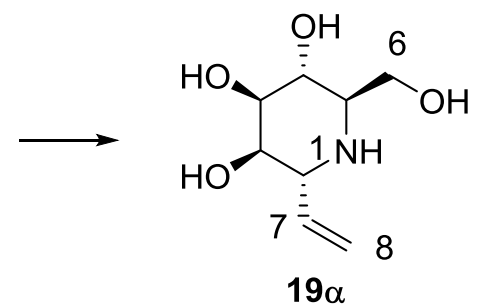

$19 \alpha$

Compound $19 \alpha$ Compound $18 \alpha(8.7 \mathrm{mg}, 0.032 \mathrm{mmol})$ was dissolved in aq $\mathrm{HCl}(5 \mathrm{~mL})$ and stirred at room temp for $4 \mathrm{~h}$. The solvent was evaporated and the residue was purified using ion-exchange chromatography (Dowex 50WX8 (200-400 mesh). The column was washed thoroughly with $\mathrm{MeOH}(25 \mathrm{~mL})$ and water $(25 \mathrm{~mL})$ followed by $1 \mathrm{M} \mathrm{NH}_{4} \mathrm{OH}(40 \mathrm{~mL})$ to give $19 \alpha(4.3 \mathrm{mg}, 70 \%)$ as a yellow foam. (1:9 MeOH:EtOH containing $1 \% \mathrm{NH}_{4} \mathrm{OH}, \mathrm{R}_{\mathrm{f}}$ 0.35); ${ }^{1} \mathrm{H}$ NMR (500 MHz, $\mathrm{D}_{2} \mathrm{O}$ ): $\delta 5.81$ (ddd, $J=17.8,10.6,5.7 \mathrm{~Hz}, 1 \mathrm{H}, \mathrm{H}-7$ ), 5.23 (apt d, $J$ 


\section{Supporting information - Experimental Section}

$=11.8 \mathrm{~Hz}, \mathrm{IH}, \mathrm{H}-8$ ), 5.23 (apt d, $J=16.6 \mathrm{~Hz}, 1 \mathrm{H}, \mathrm{H}-8^{\prime}$ ), 3.92 (t, $J=2.6 \mathrm{~Hz}, 1 \mathrm{H}, \mathrm{H}-2$ ), $3.69-$ 3.66 (m, 2H, H-6, H-6', overlapping signals), 3.60 (dd, $J=5.5,2.7 \mathrm{~Hz}, 1 \mathrm{H}, \mathrm{H}-1$ ), $3.58-3.57$ (m, 2H, H-3, H-4, overlapping signals), 2.74 (ddt, $J=7.8,5.1,3.0 \mathrm{~Hz}, 1 \mathrm{H}, \mathrm{H}-5) ;{ }^{13} \mathrm{C} \mathrm{NMR}$ (126 MHz, $\mathrm{D}_{2} \mathrm{O}$ ): $\delta 133.3$ (C-7), 118.5 (C-8), 71.9 (C-2), 71.2 (C-3), 68.1 (C-4), 60.3 (C-6), 59.0 (C-1), 55.6 (C-5). HRMS (ESI) m/z calc for $\mathrm{C}_{8} \mathrm{H}_{16} \mathrm{O}_{4} \mathrm{~N}$ : 190.1079, Found: 190.1080 [M$\mathrm{H}]^{-}$.

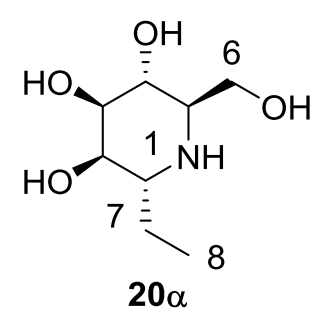

Compound 20 $\alpha$. The alkene precursor $(20 \mathrm{mg}, 0.11 \mathrm{mmol})$ was dissolved in $\mathrm{MeOH}$ (10 $\mathrm{mL})$. Pd-C (10 mol \%) and $\mathrm{HCl}(3.6 \mu \mathrm{L}, 0.12 \mathrm{mmol})$ were charged. $\mathrm{A}_{2}$ balloon was attached and the reaction was stirred at room temp for $4 \mathrm{~h}$. The reaction was filtered and the solvent was removed under diminished pressure. The residue was purified by ion exchange chromatography $\left(\mathrm{MeOH}, \mathrm{H}_{2} \mathrm{O}, 1.0 \mathrm{M} \mathrm{NH}_{4} \mathrm{OH}\right)$ to give $20 \alpha(16 \mathrm{mg}, 80 \%)$ as a clear oil; $[\alpha]_{\mathrm{D}}$ -2.2 (c 0.29, $\mathrm{D}_{2} \mathrm{O}$ ); ${ }^{1} \mathrm{H}$ NMR (500 MHz, $\left.\mathrm{D}_{2} \mathrm{O}\right) \delta 3.97$ (t, J = $\left.2.8 \mathrm{~Hz}, 1 \mathrm{H}, \mathrm{H}-2\right), 3.79$ (apt d, J = 4.0 Hz, 2H, H-6, H-6'), 3.76 (dd, J = 9.5, 3.2 Hz, 1H, H-3), 3.64 (t, J = 9.7 Hz, 1H, H-4), 2.93 $(\mathrm{td}, \mathrm{J}=7.6,2.4 \mathrm{~Hz}, 1 \mathrm{H}, \mathrm{H}-1), 2.74$ (dt, J = 9.9, 4.0 Hz, 1H, H-5), 1.59 (p, J = 7.5 Hz, 2H, $\mathrm{CH} 2), 0.97$ (t, J = 7.5 Hz, 3H, $\left.-\mathrm{CH}_{3}\right) ;{ }^{13} \mathrm{C} \mathrm{NMR}\left(126 \mathrm{MHz}, \mathrm{D}_{2} \mathrm{O}\right) \delta 71.4$ (C-3), 71.1 (C-2), 68.6 (C-4), 60.8 (C-6), 59.0 (C-1), 54.8 (C-5), $21.5\left(-\mathrm{CH}_{2}\right), 10.5\left(-\mathrm{CH}_{3}\right)$; FTIR 3301, 2964, 2934, 2881, 1664, 1613, 1572, 1461, 1404, 1344, 1250, 1150, 1054, 952, 891, 812, $749 \mathrm{~cm}^{-1}$.

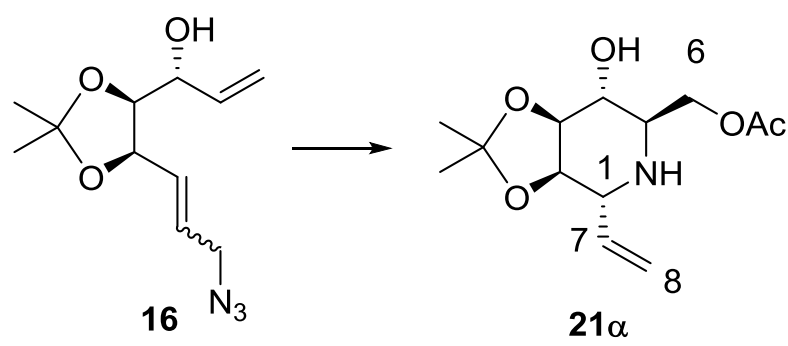

Compound 21 $\alpha$ Azide 16 (300 mg, $1.25 \mathrm{mmol}$ ) was dissolved in DMF (30 mL) and heated to $100{ }^{\circ} \mathrm{C}$ for $1 \mathrm{~h}$. The reaction was cooled to $50{ }^{\circ} \mathrm{C}$. Acetic acid $(0.36 \mathrm{~mL}, 6.27 \mathrm{mmol})$ was added and the solution was stirred at room temp for $8 \mathrm{~h}$. The mixture was diluted with $\mathrm{H}_{2} \mathrm{O}$ $(40 \mathrm{~mL})$ and extracted with EtOAc until no product remained in the aqueous layer as 


\section{Supporting information - Experimental Section}

evidenced by TLC analysis. The organic portion was dried over $\mathrm{Na}_{2} \mathrm{SO}_{4}$ and the solvent was removed. Flash column chromatography (1:1 EtOAc: hexane) gave 21 as a pale yellow foam $(162 \mathrm{mg}, 45 \%) ;[\alpha]_{\mathrm{D}}-17.6\left(c 0.47, \mathrm{CHCl}_{3}\right) ;{ }^{1} \mathrm{H} \mathrm{NMR}\left(500 \mathrm{MHz}, \mathrm{CDCl}_{3}\right) \delta 5.85$ (ddd, $J=$ 17.4, 10.6, $5.5 \mathrm{~Hz}, 1 \mathrm{H}, \mathrm{H}-7$ ), 5.31 (ddd, $J=17.4,1.7,1.1 \mathrm{~Hz}, 1 \mathrm{H}, \mathrm{H}-8$ ), 5.26 (dt, $J=10.6,1.4$ Hz, 1H, H-8'), 4.46 (dd, $J=11.4,5.8 \mathrm{~Hz}, 1 \mathrm{H}, \mathrm{H}-6$ ), 4.18 (dd, $J=11.4,3.3 \mathrm{~Hz}, 1 \mathrm{H}, \mathrm{H}-6$ '), 4.14 (dd, $J=5.6,4.1 \mathrm{~Hz}, 1 \mathrm{H}, \mathrm{H}-2), 4.05$ (dd, $J=6.7,5.6 \mathrm{~Hz}, 1 \mathrm{H}, \mathrm{H}-3), 3.72$ (ddt, $J=5.7,3.9$, $1.7 \mathrm{~Hz}, 1 \mathrm{H}, \mathrm{H}-1), 3.59$ (dd, $J=8.9,6.7 \mathrm{~Hz}, 1 \mathrm{H}, \mathrm{H}-4), 2.91$ (ddd, $J=9.0,5.8,3.3 \mathrm{~Hz}, 1 \mathrm{H}, \mathrm{H}-$ 5), $2.10\left(\mathrm{~s}, 3 \mathrm{H}, \mathrm{COCH}_{3}\right), 1.53\left(\mathrm{~s}, 3 \mathrm{H},-\mathrm{C}\left(\mathrm{CH}_{3}\right)_{2}\right), 1.38\left(\mathrm{~s}, 3 \mathrm{H},-\mathrm{C}\left(\mathrm{CH}_{3}\right)_{2}\right) ;{ }^{13} \mathrm{C} \mathrm{NMR}(126$ $\left.\mathrm{MHz}, \mathrm{CDCl}_{3}\right): \delta 171.3(\mathrm{C}=\mathrm{O}), 136.8(\mathrm{CH}, \mathrm{C}-7), 117.2\left(\mathrm{CH}_{2}, \mathrm{C}-8\right), 109.1\left(\mathrm{C},-\mathrm{C}_{\left.\left(\mathrm{CH}_{3}\right)_{2}\right), 78.6}\right.$ (CH, C-3), 76.8 (CH, C-2), 70.4 (CH, C-4), $64.0\left(\mathrm{CH}_{2}, \mathrm{C}-6\right), 55.1(\mathrm{CH}, \mathrm{C}-1), 53.2(\mathrm{CH}, \mathrm{C}-5)$, $28.1\left(\mathrm{CH}_{3}, \mathrm{C}\left(\mathrm{CH}_{3}\right)_{2}\right), 26.1\left(\mathrm{CH}_{3}, \mathrm{C}\left(\mathrm{CH}_{3}\right)_{2}\right), 20.8\left(\mathrm{CH}_{3},-\mathrm{COCH}_{3}\right)$; FTIR 3323, 2986, 2935, 1737, 1644, 1457, 1370, 1238, 1219, 1164, 1056, 924, 842, 786, $738 \mathrm{~cm}^{-1}$; HRMS (ESI) $\mathrm{m} / \mathrm{z}$ calc for $\mathrm{C}_{13} \mathrm{H}_{22} \mathrm{O}_{5} \mathrm{~N}$ : 272.1498, Found: $272.1507[\mathrm{M}+\mathrm{H}]^{+}$.

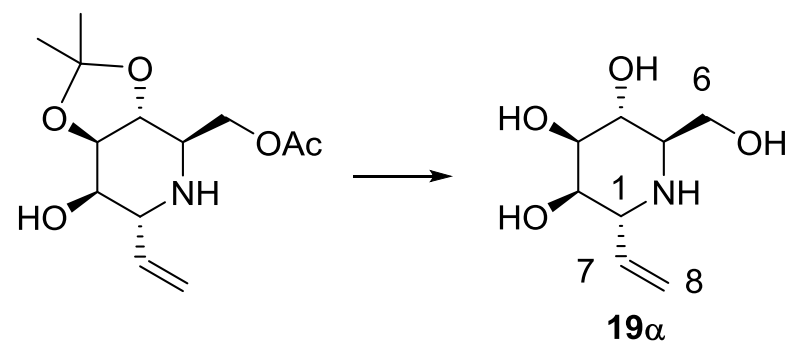

Compound $19 \alpha$ The protected precursor $(40 \mathrm{mg}, 0.15 \mathrm{mmol}$ ) was dissolved in aq $\mathrm{HCl}$ $(5 \mathrm{~mL})$ and stirred at room temp for $4 \mathrm{~h}$. The solvent was removed under diminished pressure and the residue was purified using ion-exchange chromatography (Dowex 50WX8, 200-400 mesh). The resin was washed thoroughly with $\mathrm{MeOH}(25 \mathrm{~mL})$, water $(25 \mathrm{~mL})$ followed by 1 $\mathrm{M} \mathrm{NH}_{4} \mathrm{OH}(40 \mathrm{~mL})$ to give $19 \alpha(25 \mathrm{mg}, 91 \%)$ as a yellow foam; $[\alpha]_{\mathrm{D}}+18.7(c 0.44, \mathrm{MeOH})$; ${ }^{1} \mathrm{H}$ NMR $\left(500 \mathrm{MHz}, \mathrm{D}_{2} \mathrm{O}\right) \delta 5.98$ (ddd, $J=17.7,10.5,6.0 \mathrm{~Hz}, 1 \mathrm{H}, \mathrm{H}-7$ ), 5.44 (apt d, $J=10.6$ Hz, 1H, H-8), 5.44 (apt d, $J=17.8$ Hz, 1H, H-8'), 4.11 (t, $J=2.8$ Hz, 1H, H-2), $3.890-3.83$ (m, 3H, H-1, H-6, H-6', overlapping signals), 3.81 - 3.76 (m, 2H, H-3, H-4, overlapping signals), 3.01 (m, 1H H-5); ${ }^{13} \mathrm{C}$ NMR (126 MHz, $\left.\mathrm{D}_{2} \mathrm{O}\right) \delta 131.9$ (C-7), 119.8 (C-8), 71.3 (C2), 70.8 (C-3), 67.6 (C-4), 59.7 (C-6), 59.2 (C-1), 55.8 (C-5); FTIR 3302, 2929, 2887, 2460, 1654, 1560, 1410, 1387, 1338, 1254, 1058, 928, $804 \mathrm{~cm}^{-1}$; HRMS (ESI) $\mathrm{m} / z$ calc for $\mathrm{C}_{8} \mathrm{H}_{16} \mathrm{O}_{4} \mathrm{~N}: 190.1079$, Found: $190.1084[\mathrm{M}+\mathrm{H}]^{+}$. 


\section{Supporting information - Experimental Section}

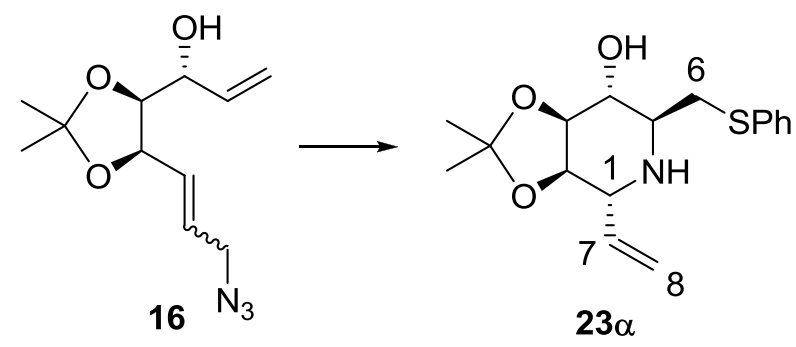

Compound 23 $\alpha$ Compound 16 (100 mg, $0.42 \mathrm{mmol}$ ) was dissolved in DMF (20 mL) and heated to $100^{\circ} \mathrm{C}$. After $1 \mathrm{hr} \mathrm{PhSH}(213 \mu \mathrm{L} 2.1 \mathrm{mmol})$ was added and solution adjusted to room temp. The reaction was then diluted with $\mathrm{H}_{2} \mathrm{O}(10 \mathrm{~mL})$ and extracted with EtOAc until no product remained in the aqueous layer. Combined organic layers were dried over $\mathrm{Na}_{2} \mathrm{SO}_{4}$ and the solvent was removed under diminished pressure. The crude residue was purified by flash chromatography (7:3, hexane-EtOAc) to give $\mathbf{2 3 \alpha}(40 \mathrm{mg}, 30 \%)$ as a yellow solid (TLC, 7:3 hexane-EtOAc, Rf, 0.1); ${ }^{1} \mathrm{H}$ NMR (500 MHz, $\left.\mathrm{CDCl}_{3}\right) \delta 7.40(\mathrm{~d}, J=8.0 \mathrm{~Hz}, 2 \mathrm{H}$, Ar-H), 7.28 (t, $J=7.9 \mathrm{~Hz}, 2 \mathrm{H}, \mathrm{Ar}-\mathrm{H}), 7.19$ (t, $J=7.3 \mathrm{~Hz}, 1 \mathrm{H}, \mathrm{Ar}-\mathrm{H}), 5.80$ (ddd, $J=16.9$, 10.5, 5.9 Hz, 1H, H-7), 5.25 (d, $J=17.3 \mathrm{~Hz}, 1 \mathrm{H}, \mathrm{H}-8), 5.19$ (d, $J=10.4 \mathrm{~Hz}, 1 \mathrm{H}, \mathrm{H}-8$ '), 4.08 (t, $J=5.1 \mathrm{~Hz}, 1 \mathrm{H}, \mathrm{H}-2), 4.02$ (t, $J=5.7 \mathrm{~Hz}, 1 \mathrm{H}, \mathrm{H}-3), 3.69$ (t, $J=6.8 \mathrm{~Hz}, 1 \mathrm{H}, \mathrm{H}-4), 3.62$ (t, $J$ $=5.2 \mathrm{~Hz}, 1 \mathrm{H}, \mathrm{H}-1), 3.39$ (dd, $J=13.9,3.6 \mathrm{~Hz}, 1 \mathrm{H}, \mathrm{H}-6), 3.15$ (dd, $J=13.7,9.1 \mathrm{~Hz}, 1 \mathrm{H}, \mathrm{H}-6$ ),

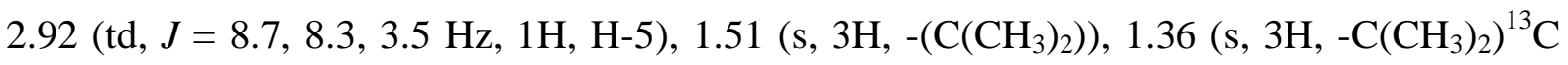
NMR (126 MHz, $\mathrm{CDCl}_{3}$ ) $\delta 137.14$ (C-7), 135.67, 130.06, 129.21, 126.63 (Ar-C), 117.25 (C8), $109.38\left(-\mathrm{C}\left(\mathrm{CH}_{3}\right)_{2}\right), 78.81$ (C-3), 76.97 (C-2), 72.60 (C-4), 55.16 (C-1), 53.52 (C-5), 36.16 (C-6), $28.29\left(-\mathrm{C}\left(\mathrm{CH}_{3}\right)_{2}\right), 26.25\left(-\mathrm{C}\left(\mathrm{CH}_{3}\right)_{2}\right)$. HRMS (ESI) m/z calc for $\mathrm{C}_{17} \mathrm{H}_{24} \mathrm{O}_{3} \mathrm{NS}: 322.1477$, found: $322.1470[\mathrm{M}+\mathrm{H}]^{+}$.
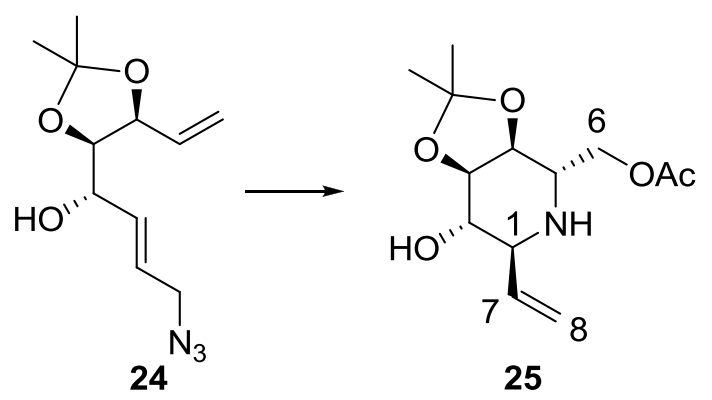

\section{Piperidine 25}

Compound 24 (200 mg, $0.84 \mathrm{mmol}$ ) was dissolved in DMF (40 mL) and heated to $100{ }^{\circ} \mathrm{C}$ for $1 \mathrm{~h}$. Acetic acid ( $24 \mu \mathrm{l}, 4.18 \mathrm{mmol})$ was added and the reaction was stirred at room temp. The reaction was diluted with $\mathrm{H}_{2} \mathrm{O}(20 \mathrm{~mL})$ and extracted with $\mathrm{Et}_{2} \mathrm{O}$ until no product remained in the aq layer (as observed by TLC 1:1 EtOAc-hexane, $\mathrm{R}_{\mathrm{f}} 0.4$ ). The combined organic layers 


\section{Supporting information - Experimental Section}

were dried over $\mathrm{Na}_{2} \mathrm{SO}_{4}$ and the solvent was removed under diminished pressure. Flash chromatography (1:1 EtOAc-hexane) gave $25(102 \mathrm{mg}, 45 \%)$ as a clear oil; $[\alpha]_{\mathrm{D}}+4.5(c 1.5$, $\left.\mathrm{CHCl}_{3}\right) ;{ }^{1} \mathrm{H}$ NMR $\left(500 \mathrm{MHz}, \mathrm{CDCl}_{3}\right) \delta 5.98(\mathrm{ddd}, J=17.3,10.3,7.1 \mathrm{~Hz}, 1 \mathrm{H}, \mathrm{H}-7), 5.29$ (dt, $J=17.2,1.3 \mathrm{~Hz}, 1 \mathrm{H}, \mathrm{H}-8), 5.24$ (dt, $J=10.3,1.2 \mathrm{~Hz}, 1 \mathrm{H}, \mathrm{H}-8$ '), 4.27 (dd, $J=11.2,4.0 \mathrm{~Hz}$, 1H, H-6), 4.13 (t, $J=6.1 \mathrm{~Hz}, 1 \mathrm{H}, \mathrm{H}-3$ ), 4.09 (dd, $J=11.2,7.4 \mathrm{~Hz}, 1 \mathrm{H}, \mathrm{H}-6$ '), 4.04 (t, $J=6.0$ $\mathrm{Hz}, 1 \mathrm{H}, \mathrm{H}-4), 3.69$ (dd, $J=7.4,6.3 \mathrm{~Hz}, 1 \mathrm{H}, \mathrm{H}-2), 3.35$ - 3.28 (m, 2H, H-1, H-5, overlapping signals), $2.09\left(\mathrm{~s}, 3 \mathrm{H},-\mathrm{OCH}_{3}\right), 1.50\left(\mathrm{~s}, 3 \mathrm{H},-\mathrm{C}\left(\mathrm{CH}_{3}\right)_{2}\right), 1.36\left(\mathrm{~s}, 3 \mathrm{H},-\mathrm{C}\left(\mathrm{CH}_{3}\right)_{2}\right) ;{ }^{13} \mathrm{C} \mathrm{NMR}(126$ $\left.\mathrm{MHz}, \mathrm{CDCl}_{3}\right) \delta 170.8(\mathrm{sC}=\mathrm{O}), 136.9(\mathrm{C}-7), 117.6(\mathrm{C}-8), 109.5\left(-\mathrm{C}\left(\mathrm{CH}_{3}\right)_{2}\right), 78.1(\mathrm{C}-3), 73.7$

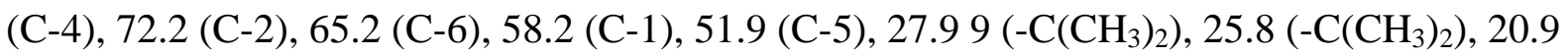
$\left(-\mathrm{OCH}_{3}\right)$; FTIR 3335, 2986, 2936, 1740, 1663, 1456, 1372, 1217, 1163, 1062, 1040, 922, 858, 787, 734, $701 \mathrm{~cm}^{-1}$; HRMS (ESI) $\mathrm{m} / z$ calc for $\mathrm{C}_{13} \mathrm{H}_{22} \mathrm{O}_{5} \mathrm{~N}: 272.1498$, Found: 272.1511 $[\mathrm{M}+\mathrm{H}]^{+}$.
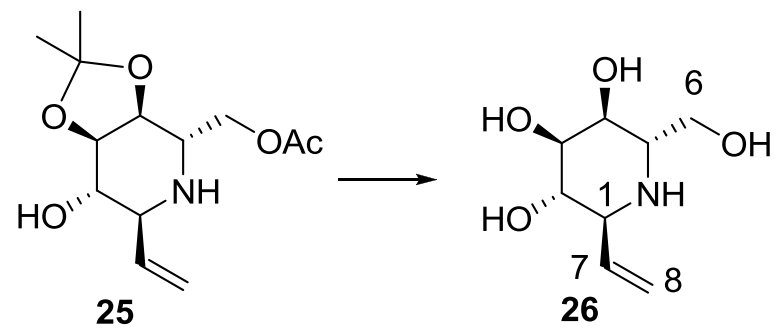

Piperidine 26 Compound 25 (97.1 mg, $0.36 \mathrm{mmol})$ was dissolved in aq $\mathrm{HCl}(5 \mathrm{~mL})$ and stirred at room temp. The reaction was the solvent was removed under diminished pressure to give a brown foam. Subsequent ion exchange chromatography $\left(\mathrm{MeOH}, \mathrm{H}_{2} \mathrm{O}\right.$ followed by $1 \mathrm{M}$ $\left.\mathrm{NH}_{4} \mathrm{OH}\right)$ gave $26(56 \mathrm{mg}, 82 \%)$ as a pale yellow foam; $[\alpha]_{\mathrm{D}}+13.1\left(\right.$ c 2.2, $\left.\mathrm{D}_{2} \mathrm{O}\right) ;{ }^{1} \mathrm{H}$ NMR $\left(500 \mathrm{MHz}, \mathrm{D}_{2} \mathrm{O}\right) \delta 5.72(\mathrm{ddd}, J=17.1,10.4,7.7 \mathrm{~Hz}, 1 \mathrm{H}, \mathrm{H}-7), 5.23$ (d, $J=17.3 \mathrm{~Hz}, 1 \mathrm{H}, \mathrm{H}-$ 8), 5.20 (d, $J=10.7 \mathrm{~Hz}, 1 \mathrm{H}, \mathrm{H}-8^{\prime}$ ), 3.92 (t, $\left.J=3.1 \mathrm{~Hz}, 1 \mathrm{H}, \mathrm{H}-4\right), 3.65$ (dd, $J=11.8,7.9 \mathrm{~Hz}$, 1H, H-6), 3.60 (dd, $J=11.8,6.4$ Hz, 1H, H-6'), 3.57 (dd, $J=9.2,3.3 \mathrm{~Hz}, 1 \mathrm{H}, \mathrm{H}-3), 3.48$ (t, $J$ $=9.1 \mathrm{~Hz}, 1 \mathrm{H}, \mathrm{H}-2), 3.15$ (apt t $, J=8.4 \mathrm{~Hz}, 1 \mathrm{H}, \mathrm{H}-1), 3.09$ (ddd, $J=7.8,6.4,2.9 \mathrm{~Hz}, 1 \mathrm{H}, \mathrm{H}-$ 5); ${ }^{13} \mathrm{C}$ NMR (126 MHz, D $\left.2 \mathrm{O}\right) \delta 134.9$ (C-7), 119.6 (C-8), 71.1 (C-3), 70.9 (C-2), 68.5 (C-4), 58.8 (C-6), 58.7 (C-5), 58.0 (C-1); FTIR 3295, 2902, 2457, 1645, 1561, 1416, 1342, 1255, 1043, 925, 843, $777 \mathrm{~cm}^{-1}$; HRMS (ESI) $\mathrm{m} / z$ calc for $\mathrm{C}_{8} \mathrm{H}_{14} \mathrm{O}_{4} \mathrm{~N}$ : 188.0923 , Found: 188.0919 $[\mathrm{M}-\mathrm{H}]^{-}$.

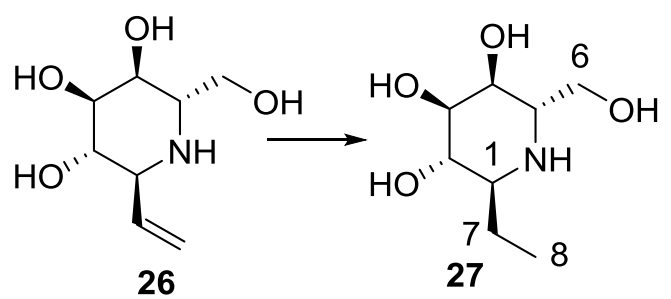




\section{Supporting information - Experimental Section}

Piperidine 27 Compound 26 (10 mg, $0.06 \mathrm{mmol}$ ) was dissolved in $\mathrm{MeOH}(5 \mathrm{~mL})$ and $\mathrm{Pd} / \mathrm{C}$ $(10 \mathrm{~mol} \%)$ and $\mathrm{HCl}(1.8 \mu \mathrm{L}, 0.06 \mathrm{mmol})$ were charged. $\mathrm{A}_{2}$ balloon was attached and the reaction was stirred under $\mathrm{H}_{2}$ at room temp for $4 \mathrm{~h}$. The reaction was filtered and the solvent was removed under diminished pressure. The residue was purified by ion exchange chromatography $\left(\mathrm{MeOH}, \mathrm{H}_{2} \mathrm{O}, 1.0 \mathrm{M} \mathrm{NH}_{4} \mathrm{OH}\right)$ to give $27(8.1 \mathrm{mg}, 80 \%)$ as a clear oil; $[\alpha]_{\mathrm{D}}$ 17.5 (c 0.63, MeOH); ${ }^{1} \mathrm{H}$ NMR (500 MHz, D $\left.2 \mathrm{O}\right) \delta 3.90$ (t, $\left.J=3.1 \mathrm{~Hz}, 1 \mathrm{H}, \mathrm{H}-4\right), 3.65-3.57$ (m, 2H, H-6, H-6', overlapping signals), 3.54 (dd, $J=9.1,3.3 \mathrm{~Hz}, 1 \mathrm{H}, \mathrm{H}-3), 3.43$ (t, $J=9.1$ Hz, 1H, H-2), $3.10-3.06$ (m, 1H, H-5), 2.55 (td, $J=8.5,3.8$ Hz, 1H, H-1), 1.73 (dqd, $J=$ 15.2, 7.8, 4.1 Hz, 1H, H-7), 1.36 (dp, $J=14.8,7.5 \mathrm{~Hz}, 1 \mathrm{H}, \mathrm{H}-7$ '), 0.83 (t, $J=7.6 \mathrm{~Hz}, 3 \mathrm{H}$, $\left.\mathrm{CH}_{3}\right) ;{ }^{13} \mathrm{C}$ NMR (126 MHz, D $\left.2 \mathrm{O}\right) \delta 71.4(\mathrm{C}-3), 70.8$ (C-2), 68.4 (C-4), 58.7 (C-5), 58.6 (C-6), 55.7 (C-1), 23.1 (C-7), 8.9 (C-8); HRMS (ESI) $m / z$ calc for $\mathrm{C}_{8} \mathrm{H}_{18} \mathrm{O}_{4} \mathrm{~N}$ : 192.1236, Found: $192.1232[\mathrm{M}+\mathrm{H}]^{+}$.
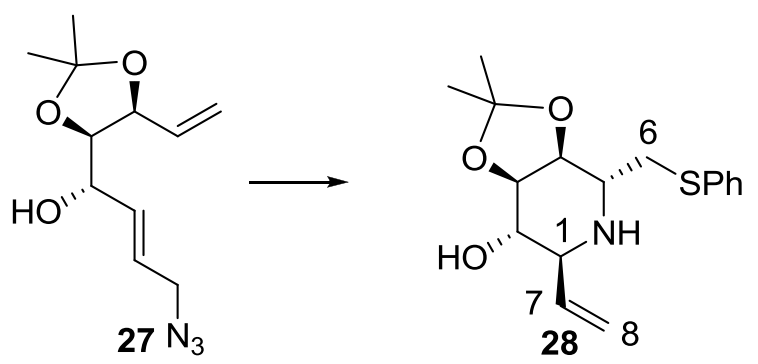

Piperidine 28 Compound 27 (120 mg, $0.50 \mathrm{mmol})$ was dissolved in DMF (2.5 mL) and heated to $100{ }^{\circ} \mathrm{C}$ for $1 \mathrm{~h}$. Thiophenol $(0.26 \mathrm{~mL}, 2.51 \mathrm{mmol})$ was added and the reaction was stirred at room temp. The reaction was diluted with $\mathrm{H}_{2} \mathrm{O}(5 \mathrm{~mL})$ and extracted with $\mathrm{Et}_{2} \mathrm{O}$ until no product remained in the aq. layer. The combined organic layers were dried over $\mathrm{Na}_{2} \mathrm{SO}_{4}$ and the solvent was removed under diminished pressure. Flash column chromatography $(2: 3$ EtOAc-hexane with 2\% $\left.\mathrm{NH}_{3}\right)$ gave $28(64 \mathrm{mg}, 40 \%)$ as a clear oil (TLC 2:3 EtOAc-hexane, $\mathrm{R}_{\mathrm{f}}$ 0.2); ${ }^{1} \mathrm{H}$ NMR (500 MHz, $\left.\mathrm{CDCl}_{3}\right) \delta 5.94(\mathrm{ddd}, J=17.4,10.3,7.1 \mathrm{~Hz}, 1 \mathrm{H}, \mathrm{H}-7), 5.24(\mathrm{~d}, J$ $=17.3 \mathrm{~Hz}, 1 \mathrm{H}, \mathrm{H}-8), 5.18$ (d, $J=10.4 \mathrm{~Hz}, 1 \mathrm{H}, \mathrm{H}-8$ '), 4.15 (t, $J=5.7 \mathrm{~Hz}, 1 \mathrm{H}, \mathrm{H}-3), 4.02$ (t, $J$ $=6.1 \mathrm{~Hz}, 1 \mathrm{H}, \mathrm{H}-4), 3.72$ (t, $J=6.1 \mathrm{~Hz}, 1 \mathrm{H}, \mathrm{H}-2), 3.37$ (dd, $J=13.6,3.6 \mathrm{~Hz}, 1 \mathrm{H}, \mathrm{H}-6$ '), 3.30 $(\mathrm{t}, J=6.8 \mathrm{~Hz}, 1 \mathrm{H}, \mathrm{H}-1), 3.13(\mathrm{ddd}, J=9.9,6.5,3.7 \mathrm{~Hz}, 1 \mathrm{H}, \mathrm{H}-5), 2.80$ (dd, $J=13.6,9.8 \mathrm{~Hz}$, $1 \mathrm{H}, \mathrm{H}-6), 1.40\left(\mathrm{~s}, 3 \mathrm{H},-\mathrm{C}\left(\mathrm{CH}_{3}\right)_{2}\right), 1.35\left(\mathrm{~s}, 3 \mathrm{H},-\mathrm{C}\left(\mathrm{CH}_{3}\right)_{2}\right) ;{ }^{13} \mathrm{C} \mathrm{NMR}\left(126 \mathrm{MHz}, \mathrm{CDCl}_{3}\right) \delta$ 136.6 (C-7), 134.7, 130.1, 129.0, 126.6 (Ar-C), 117.5 (C-8), $109.5\left(-\mathrm{C}\left(\mathrm{CH}_{3}\right)_{2}\right), 77.8$ (C-3), 76.3 (C-4), 71.7 (C-2), 58.5 (C-1), 51.2 (C-5), $37.3(\mathrm{C}-6), 27.7\left(-\mathrm{C}\left(\mathrm{CH}_{3}\right)_{2}\right), 25.9\left(-\mathrm{C}\left(\mathrm{CH}_{3}\right)_{2}\right)$; HRMS (ESI) m/z calc for $\mathrm{C}_{17} \mathrm{H}_{24} \mathrm{O}_{3} \mathrm{NS}: 322.1477$, found: $322.1469[\mathrm{M}+\mathrm{H}]^{+}$. 


\section{Supporting information - Experimental Section}
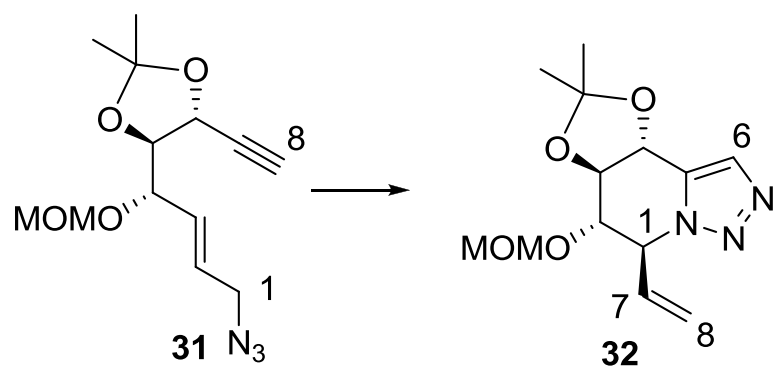

Triazole 32 Compound 31 (47 mg, $0.17 \mathrm{mmol}$ ) was dissolved in anhydrous DMF (5 mL) and heated to $100{ }^{\circ} \mathrm{C}$ for $8 \mathrm{~h}$. The mixture was cooled to room temp. Water $(5 \mathrm{~mL})$ was added and the layers were separated. The aq layer was extracted with EtOAc $(3 \times 10 \mathrm{~mL})$ and the combined organic layers were dried over $\mathrm{Na}_{2} \mathrm{SO}_{4}$, filtered and the solvent was removed under diminished pressure. Flash column chromatography of the residue (1:4 EtOAc-hexane) gave 32 (26 mg, $63 \%$ ) as a white solid. (TLC 1:1 EtOAc-hexane, $\mathrm{R}_{\mathrm{f}} 0.7$ ); $[\alpha]_{\mathrm{D}}-64.3$ (c 1.2, $\left.\mathrm{CHCl}_{3}\right) ;{ }^{1} \mathrm{H}$ NMR $\left(500 \mathrm{MHz}, \mathrm{CDCl}_{3}\right) \delta 7.69$ (d, $\left.J=1.0 \mathrm{~Hz}, 1 \mathrm{H}, \mathrm{H}-6\right), 6.04$ (ddd, $J=17.0$, 10.3, 6.9 Hz, 1H, H-7), 5.42 (dd, $J=10.3,1.1 \mathrm{~Hz}, 1 \mathrm{H}, \mathrm{H}-8), 5.25(\mathrm{dd}, J=16.9,1.2 \mathrm{~Hz}, 1 \mathrm{H}$, H-8'), 5.06 (t, $J=6.6 \mathrm{~Hz}, 1 \mathrm{H}, \mathrm{H}-1), 5.02$ (d, $\left.J=6.8 \mathrm{~Hz}, 1 \mathrm{H},-\mathrm{OCH}_{2} \mathrm{O}-\right), 4.72$ (d, $J=6.8 \mathrm{~Hz}$, $\left.1 \mathrm{H},-\mathrm{OCH}_{2} \mathrm{O}-\right), 4.60$ (d, $\left.J=9.2 \mathrm{~Hz}, 1 \mathrm{H}, \mathrm{H}-4\right), 4.30$ (dd, $\left.J=9.9,6.3 \mathrm{~Hz}, 1 \mathrm{H}, \mathrm{H}-2\right), 3.81$ (t, $J=$ $9.6 \mathrm{~Hz}, 1 \mathrm{H}, \mathrm{H}-3), 3.44$ (s, 3H, $\left.-\mathrm{OCH}_{3}\right), 1.55$ (s, $\left.3 \mathrm{H},-\mathrm{C}\left(\mathrm{CH}_{3}\right)_{2}\right), 1.50\left(\mathrm{~s}, 3 \mathrm{H},-\mathrm{C}\left(\mathrm{CH}_{3}\right)_{2}\right)$; ${ }^{13} \mathrm{C}$ NMR (126 MHz, CDCl ${ }_{3}$ ) $\delta 133.8$ (C-7), 133.2 (C-5), 128.3 (C-6), 119.7 (C-8), 114.6 ($\left.\mathrm{C}\left(\mathrm{CH}_{3}\right)_{2}\right), 96.0\left(-\mathrm{OCH}_{2} \mathrm{O}-\right), 80.7$ (C-3), 75.4 (C-2), $70.1(\mathrm{C}-4), 65.8(\mathrm{C}-1), 56.1\left(-\mathrm{OCH}_{3}\right), 26.8$ $\left(-\mathrm{C}\left(\mathrm{CH}_{3}\right)_{2}\right), 26.6\left(-\mathrm{C}\left(\mathrm{CH}_{3}\right)_{2}\right)$; FTIR 2998, 2951, 2885, 1646, 1421, 1381, 1296, 1228, 1195 , 1152, 1102, 1067, 1048, 1019, 984, 964, 947, 912, 881, 847, 810, 775, $679 \mathrm{~cm}^{-1}$; HRMS (ESI) $m / z$ calc for $\mathrm{C}_{13} \mathrm{H}_{20} \mathrm{~N}_{3} \mathrm{O}_{4}$ : 282.1454: found $282.1441[\mathrm{M}+\mathrm{H}]^{+}$.

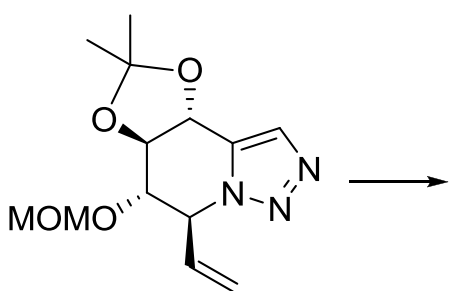

32

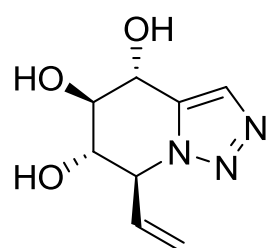

33

Compound 33 Compound 32 (26 mg, $0.09 \mathrm{mmol})$ was dissolved in methanolic $\mathrm{HCl}(5 \mathrm{~mL})$ and stirred at room temp overnight. The solvent was removed under diminished pressure to give 33 was obtained as a pale yellow foam $(18 \mathrm{mg},>90 \%)$. $[\alpha]_{\mathrm{D}}+23.6\left(c 3.9, \mathrm{D}_{2} \mathrm{O}\right) ;{ }^{1} \mathrm{H}$ NMR (500 MHz, CD 3 OD) $\delta 8.39$ (s, 1H, H-6), 5.92 (ddd, $J=17.0,10.1,8.3 \mathrm{~Hz}, 1 \mathrm{H}, \mathrm{H}-7)$, $5.54(\mathrm{~d}, J=17.0 \mathrm{~Hz}, 1 \mathrm{H}, \mathrm{H}-8), 5.50$ (d, $J=10.0 \mathrm{~Hz}, 1 \mathrm{H}, \mathrm{H}-8$ '), 4.83 (t, $J=8.3 \mathrm{~Hz}, 1 \mathrm{H}, \mathrm{H}-1)$, 4.71 (d, $J=7.9 \mathrm{~Hz}, 1 \mathrm{H}, \mathrm{H}-4), 3.82$ (dd, $J=9.3,8.4 \mathrm{~Hz}, 1 \mathrm{H}, \mathrm{H}-2), 3.75$ (dd, $J=9.3,7.9 \mathrm{~Hz}$, 1H, H-3); ${ }^{13} \mathrm{C}$ NMR (126 MHz, CD $\left.{ }_{3} \mathrm{OD}\right) \delta 141.4$ (C-5), 131.4 (C-7), 127.4 (C-6), 122.6 (C- 


\section{Supporting information - Experimental Section}

8), 74.2 (C-3), 70.8 (C-2), 67.7 (C-1), 65.9 (C-4); FTIR 3266, 2451, 1914, 1679, 1578, 1422, 1378, 1306, 1276, 1209, 1088, 1060, 1009, 933, 835, 794, $673 \mathrm{~cm}^{-1}$; HRMS (ESI) m/z calc for $\mathrm{C}_{8} \mathrm{H}_{12} \mathrm{~N}_{3} \mathrm{O}_{3}$ : 198.0879: found 198.0878 [M+H] $]^{+}$.

Supplementary Scheme 1: Synthesis of 31

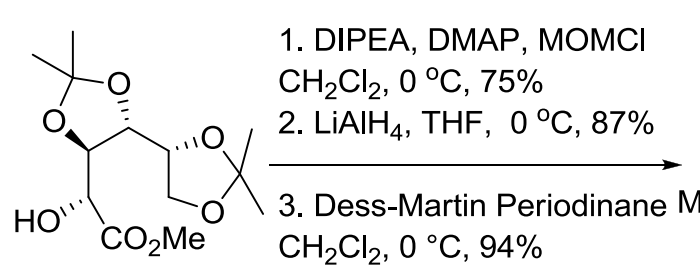

S1

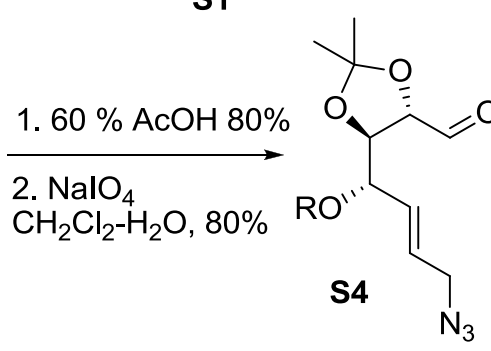

Supplementary Scheme 2: Synthesis of 24

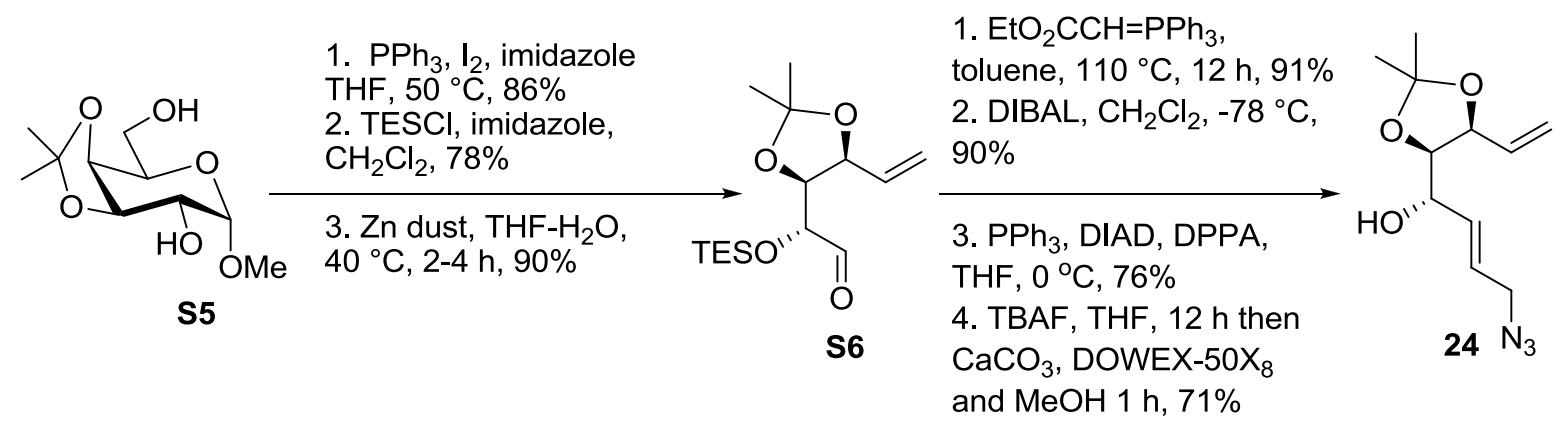

1. VinylMgBr, THF $0^{\circ} \mathrm{C}, 50-65 \%$ 2. $\mathrm{MsCl}, \mathrm{Et}_{3} \mathrm{~N}$ $\mathrm{CH}_{2} \mathrm{Cl}_{2}, 0^{\circ} \mathrm{C}, 86 \%$ 3. $\mathrm{NaN}_{3}, \mathrm{DMF}$ $100{ }^{\circ} \mathrm{C}, 79 \%$

S2<smiles>C#CC1OC(C)(C)O[C@H]1[C@H](/C=C/CN)OC</smiles><smiles>CO[C@H](/C=C/CN)[C@H]1OC(C)(C)O[C@H]1[C@H]1COC(C)(C)O1</smiles>

\section{Synthetic route to 31}<smiles></smiles>

S1<smiles>CO[C@H](C=O)[C@H]1OC(C)(C)O[C@H]1[C@H]1COC(C)(C)O1</smiles>

S2

Compound S2. $\quad$ Ester $\mathbf{S 1}^{3}(8.0 \mathrm{~g}, 0.028 \mathrm{~mol})$ in $\mathrm{CH}_{2} \mathrm{Cl}_{2}(150 \mathrm{~mL})$ was cooled to $0{ }^{\circ} \mathrm{C}$. $\mathrm{N}, \mathrm{N}$-Diisopropylethylamine $(49.2 \mathrm{~mL}, 0.28 \mathrm{~mol})$ and DMAP $(1.68 \mathrm{~g}, 0.014 \mathrm{~mol})$ were charged. $\mathrm{MOMCl}(20.9 \mathrm{~mL}, 0.28 \mathrm{~mol})$ was charged over $2 \mathrm{~h}$. The reaction was allowed to 


\section{Supporting information - Experimental Section}

reach room temp and stirred for $48 \mathrm{~h}$. The reaction was diluted with $\mathrm{CH}_{2} \mathrm{Cl}_{2}(100 \mathrm{~mL})$, washed with $2 \% \mathrm{HCl}(50 \mathrm{~mL}), \mathrm{NaHCO}_{3}(50 \mathrm{~mL})$, brine $(50 \mathrm{~mL})$, dried over $\mathrm{Na}_{2} \mathrm{SO}_{4}$ and the solvent was removed to give the MOM derivative as a yellow solid (7.5 g, $75 \%)$; $\mathrm{R}_{\mathrm{f}}$ on TLC 0.8, 1:2 EtOAc-hexane, $) ;[\alpha]_{\mathrm{D}}+59\left(c 1.4, \mathrm{CHCl}_{3}\right) ;{ }^{1} \mathrm{H} \mathrm{NMR}\left(500 \mathrm{MHz}, \mathrm{CDCl}_{3}\right) \delta 4.78(\mathrm{~d}, J=$ $\left.6.8 \mathrm{~Hz}, 1 \mathrm{H},-\mathrm{OCH}_{2} \mathrm{O}-\right), 4.74\left(\mathrm{~d}, J=6.8 \mathrm{~Hz}, 1 \mathrm{H},-\mathrm{OCH}_{2} \mathrm{O}-\right), 4.38(\mathrm{dd}, J=7.1,2.7 \mathrm{~Hz}, 1 \mathrm{H}, \mathrm{H}-$ 4), 4.35 (d, $J=2.6 \mathrm{~Hz}, 1 \mathrm{H}, \mathrm{H}-2), 4.18$ (dd, $J=8.5,6.2 \mathrm{~Hz}, 1 \mathrm{H}, \mathrm{H}-6), 4.08$ (dt, $J=8.4,6.0 \mathrm{~Hz}$, 1H, H-5), 4.03 - 3.98 (m, 1H, H-3), 3.92 (dd, $J=8.5,5.6$ Hz, 1H, H-6'), 3.80 (s, 3H, $\left.\mathrm{COOCH}_{3}\right), 3.44\left(\mathrm{~s}, 3 \mathrm{H},-\mathrm{OCH}_{2} \mathrm{OCH}_{3}\right), 1.43\left(\mathrm{~s}, 3 \mathrm{H},-\mathrm{C}\left(\mathrm{CH}_{3}\right)_{2}\right), 1.41\left(\mathrm{~s}, 3 \mathrm{H},-\mathrm{C}\left(\mathrm{CH}_{3}\right)_{2}\right), 1.37$ (s, 3H, - $\left.\mathrm{C}\left(\mathrm{CH}_{3}\right)_{2}\right), 1.35\left(\mathrm{~s}, 3 \mathrm{H},-\mathrm{C}\left(\mathrm{CH}_{3}\right)_{2}\right) ;{ }^{13} \mathrm{C} \mathrm{NMR}\left(126 \mathrm{MHz}, \mathrm{CDCl}_{3}\right) \delta 170.5(\mathrm{C}-1), 110.3$ $\left(-\mathrm{C}\left(\mathrm{CH}_{3}\right)_{2}\right), 109.8\left(-\mathrm{C}\left(\mathrm{CH}_{3}\right)_{2}\right), 96.7$ (-OCH $\left.2 \mathrm{O}-\right), 80.7$ (C-4), 77.1, 77.1 (C-3, C-5), 74.6 (C-2), $67.9(\mathrm{C}-6), 56.6\left(-\mathrm{OCH}_{2} \mathrm{OCH}_{3}\right), 52.2\left(-\mathrm{COOCH}_{3}\right), 27.2\left(-\mathrm{C}\left(\mathrm{CH}_{3}\right)_{2}\right), 26.6\left(-\mathrm{C}\left(\mathrm{CH}_{3}\right)_{2}\right), 26.4(-$ $\left.\mathrm{C}\left(\mathrm{CH}_{3}\right)_{2}\right), 25.1\left(-\mathrm{C}\left(\mathrm{CH}_{3}\right)_{2}\right)$; FTIR 2986, 2960, 2935, 1750, 1433, 1383, 1372, 1252, 1210 , 1149, 1061, 1022, 981, 920, 845, $797 \mathrm{~cm}^{-1}$; HRMS (ESI) $\mathrm{m} / z$ calc $\mathrm{C}_{15} \mathrm{H}_{26} \mathrm{O}_{8} \mathrm{Na}: 357.1525$, found: $357.1532[\mathrm{M}+\mathrm{Na}]^{+}$. Next a $1.0 \mathrm{M}$ soln of $\mathrm{LiAlH}_{4}(2.05 \mathrm{~g}, 0.05 \mathrm{~mol})$ in THF $(50 \mathrm{~mL})$ was cooled to $0{ }^{\circ} \mathrm{C}$. A solution of the MOM protected derivative of $\mathbf{S 1}(12.0 \mathrm{~g}, 0.04 \mathrm{~mol})$ in THF $(100 \mathrm{~mL})$ was added dropwise, maintaining the temperature at $0{ }^{\circ} \mathrm{C}$. The reaction was then stirred at room temp for $3 \mathrm{~h}$ and was then quenched at $0{ }^{\circ} \mathrm{C}$ with aq $\mathrm{Na}_{2} \mathrm{SO}_{4}$, filtered and washed with EtOAc $(3 \times 50 \mathrm{~mL})$. The organic layer was washed with brine $(50 \mathrm{~mL})$, dried over $\mathrm{Na}_{2} \mathrm{SO}_{4}$ and the solvent was removed to give the primary alcohol intermediate as a clear oil (11 g, $87 \%)$; $[\alpha]_{\mathrm{D}}-10\left(c 1.5, \mathrm{CHCl}_{3}\right) ;{ }^{1} \mathrm{H}$ NMR $\left(500 \mathrm{MHz}, \mathrm{CDCl}_{3}\right) \delta 4.82(\mathrm{~d}, J=6.7 \mathrm{~Hz}$, $\left.1 \mathrm{H},-\mathrm{OCH}_{2} \mathrm{O}-\right), 4.74$ (d, $\left.J=6.8 \mathrm{~Hz}, 1 \mathrm{H},-\mathrm{OCH}_{2} \mathrm{O}-\right), 4.18$ (dd, $\left.J=8.5,6.2 \mathrm{~Hz}, 1 \mathrm{H}, \mathrm{H}-6\right), 4.14$ - 4.03 (m, 2H, H-3, H-4), 3.96 - 3.92 (m, 2H, H-5, H-6'), 3.80 - 3.78 (m, 2H, - $\mathrm{CH}_{2} \mathrm{OH}$ ), 3.74 - $3.71(\mathrm{~m}, 1 \mathrm{H}, \mathrm{H}-2), 3.46\left(\mathrm{~s}, 3 \mathrm{H},-\mathrm{OCH}_{3}\right), 1.41\left(\mathrm{~s}, 6 \mathrm{H},-\mathrm{C}\left(\mathrm{CH}_{3}\right)_{2}\right), 1.38\left(\mathrm{~s}, 3 \mathrm{H},-\mathrm{C}\left(\mathrm{CH}_{3}\right)_{2}\right)$,

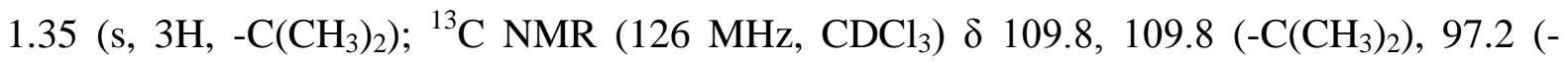
$\left.\mathrm{OCH}_{2} \mathrm{O}-\right), 81.4$ (C-4), 79.9 (C-2), 77.5, 77.4 (C-3, C-5), 68.0 (C-6), 63.5 (C-1), $55.9\left(-\mathrm{OCH}_{3}\right)$, $27.2\left(-\mathrm{C}\left(\mathrm{CH}_{3}\right)_{2}\right), 26.7\left(-\mathrm{C}\left(\mathrm{CH}_{3}\right)_{2}\right), 26.4\left(-\mathrm{C}\left(\mathrm{CH}_{3}\right)_{2}\right), 25.2\left(-\mathrm{C}\left(\mathrm{CH}_{3}\right)_{2}\right)$; FTIR 3467, 2987, 2937 , 2896, 1704, 1457, 1371, 1249, 1212, 1151, 1060, 1022, 917, 878, $845 \mathrm{~cm}^{-1}$; HRMS (ESI) $\mathrm{m} / \mathrm{z}$ calc for $\mathrm{C}_{14} \mathrm{H}_{26} \mathrm{O}_{7} \mathrm{Na}$ : 329.1576, found: $329.1591[\mathrm{M}+\mathrm{Na}]^{+}$. To a stirred solution of the DessMartin periodinane $(22.8 \mathrm{~g}, 0.05 \mathrm{~mol}$, prepared in situ $)$ in $\mathrm{CH}_{2} \mathrm{Cl}_{2}(50 \mathrm{~mL})$ at $0{ }^{\circ} \mathrm{C}$ was added a solution of the intermediate primary alcohol $(11.0 \mathrm{~g}, 0.04 \mathrm{~mol})$ in $\mathrm{CH}_{2} \mathrm{Cl}_{2}(0.35 \mathrm{M}$ solution). The suspension was stirred for $30 \mathrm{~min}$ at $0{ }^{\circ} \mathrm{C}$ and then allowed to attain room temp for $1 \mathrm{~h}$. The reaction mixture was diluted with $\mathrm{Et}_{2} \mathrm{O}(200 \mathrm{~mL})$ and poured into cold sat aq $\mathrm{NaHCO}_{3}$ (100 mL) containing $\mathrm{Na}_{2} \mathrm{~S}_{2} \mathrm{O}_{3}$ and was stirred for $30 \mathrm{~min}$. The organic layer was washed with sat aq $\mathrm{NaHCO}_{3}(50 \mathrm{~mL})$ and brine $(50 \mathrm{~mL})$, dried over $\mathrm{Na}_{2} \mathrm{SO}_{4}$, filtered and the solvent was 


\section{Supporting information - Experimental Section}

removed under reduced pressure to give $\mathbf{S 2}(9.1 \mathrm{~g}, 83 \%)$ as a colourless oil; $[\alpha]_{\mathrm{D}}+15.9(c$ 4.3, $\left.\mathrm{CHCl}_{3}\right) ;{ }^{1} \mathrm{H} \mathrm{NMR}\left(500 \mathrm{MHz}, \mathrm{CDCl}_{3}\right) \delta 9.73(\mathrm{~d}, J=1.5 \mathrm{~Hz}, 1 \mathrm{H}, \mathrm{H}-1), 4.82(\mathrm{~d}, J=6.8 \mathrm{~Hz}$, $\left.1 \mathrm{H},-\mathrm{OCH}_{2} \mathrm{O}-\right), 4.76\left(\mathrm{~d}, J=6.8 \mathrm{~Hz}, 1 \mathrm{H},-\mathrm{OCH}_{2} \mathrm{O}-\right), 4.33(\mathrm{dd}, J=7.7,2.5 \mathrm{~Hz}, 1 \mathrm{H}, \mathrm{H}-4), 4.18$ $(\mathrm{dd}, J=8.6,6.2 \mathrm{~Hz}, 1 \mathrm{H}, \mathrm{H}-6), 4.10$ (t, $J=2.0 \mathrm{~Hz}, 1 \mathrm{H}, \mathrm{H}-2), 4.06$ (dt, $J=8.7,5.7 \mathrm{~Hz}, 1 \mathrm{H}, \mathrm{H}-$ 5), 3.98 (m, 1H, H-3), 3.93 (dd, $J=8.6,5.4$ Hz, 1H, H-6'), 3.46 (s, 3H, -C(CH$\left.)_{2}\right), 1.43$ (s, $\left.3 \mathrm{H},-\mathrm{C}\left(\mathrm{CH}_{3}\right)_{2}\right), 1.40\left(\mathrm{~s}, 3 \mathrm{H},-\mathrm{C}\left(\mathrm{CH}_{3}\right)_{2}\right), 1.36\left(\mathrm{~s}, 3 \mathrm{H},-\mathrm{C}\left(\mathrm{CH}_{3}\right)_{2}\right), 1.34\left(\mathrm{~s}, 3 \mathrm{H},-\mathrm{C}\left(\mathrm{CH}_{3}\right)_{2}\right)$; ${ }^{13} \mathrm{C}$ NMR $\left(126 \mathrm{MHz}, \mathrm{CDCl}_{3}\right) \delta 201.7(\mathrm{C}-1), 110.3,109.8\left(-\mathrm{C}\left(\mathrm{CH}_{3}\right)_{2}\right), 97.6\left(-\mathrm{OCH}_{2} \mathrm{O}-\right), 81.7(\mathrm{C}-$ 2), $79.9(\mathrm{C}-4), 77.1,76.7$ (C-3, C-5), $68.0(\mathrm{C}-6), 56.4\left(-\mathrm{OCH}_{3}\right), 27.0\left(-\mathrm{C}\left(\mathrm{CH}_{3}\right)_{2}\right), 26.5$ ($\left.\mathrm{C}\left(\mathrm{CH}_{3}\right)_{2}\right), 26.4\left(-\mathrm{C}\left(\mathrm{CH}_{3}\right)_{2}\right), 25.0\left(-\mathrm{C}\left(\mathrm{CH}_{3}\right)_{2}\right)$; FTIR 2988, 2940, 2901, 1736, 1706, 1457 , $1372,1245,1213,1151,1062,1029,918,844 \mathrm{~cm}^{-1}$; HRMS (ESI) $\mathrm{m} / z$ calc for $\mathrm{C}_{14} \mathrm{H}_{24} \mathrm{O}_{7} \mathrm{Na}$ : 327.1420, found: $327.1418[\mathrm{M}+\mathrm{Na}]^{+}$.

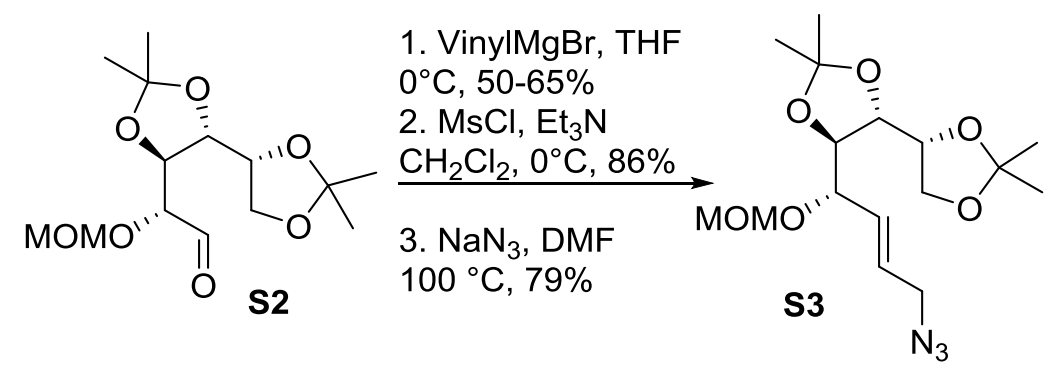

Compound S3. Compound S2 $(9.1 \mathrm{~g}, 0.03 \mathrm{~mol})$ was dissolved in THF (100 mL) and cooled to $-78{ }^{\circ} \mathrm{C}$. Vinyl magnesium bromide (44 mL, $0.04 \mathrm{~mol}, 1.0 \mathrm{M}$ in THF) was charged slowly. The reaction was stirred for $6 \mathrm{~h}$. The reaction was warmed to room temp and quenched with $\mathrm{NH}_{4} \mathrm{Cl}$ solution. The organic layer was washed with brine, dried over $\mathrm{Na}_{2} \mathrm{SO}_{4}$ and the solvent was removed under reduced pressure. The crude mixture was purified by flash column chromatography (EtOAc-hexane 1:1) to give a mixture of isomeric alcohols $(\sim 1: 1,5.5 \mathrm{~g}, 56 \%)$; Rf by TLC 0.55; 1:2 EtOAc-hexane); ${ }^{1} \mathrm{H}$ NMR (500 MHz, $\left.\mathrm{CDCl}_{3}\right) \delta 6.03$ (ddd, $J=17.2,10.5,4.8 \mathrm{~Hz}, 1 \mathrm{H}, \mathrm{C}-2), 5.94$ (ddd, $J=16.9,10.6,5.9 \mathrm{~Hz}, 1 \mathrm{H}, \mathrm{C}-2), 5.44$ (ddt, $J=17.3,8.3,1.8 \mathrm{~Hz}, 2 \mathrm{H}, \mathrm{C}-1^{\prime}$ '), 5.23 (dt, $J=10.4,1.8 \mathrm{~Hz}, 2 \mathrm{H}, \mathrm{C}-1$ '), 4.80 (apt t, $J=6.9 \mathrm{~Hz}$, $\left.2 \mathrm{H},-\mathrm{OCH}_{2} \mathrm{O}-\right), 4.76$ (d, $\left.J=6.7 \mathrm{~Hz}, 1 \mathrm{H},-\mathrm{OCH}_{2} \mathrm{O}-\right), 4.71\left(\mathrm{~d}, J=6.8 \mathrm{~Hz}, 1 \mathrm{H},-\mathrm{OCH}_{2} \mathrm{O}-\right), 4.42$ $-3.45\left(\mathrm{~m}, 14 \mathrm{H}, 12 \mathrm{x}-\mathrm{CH}, 2 \mathrm{x}-\mathrm{CH}_{2}\right), 3.45\left(\mathrm{~s}, 3 \mathrm{H},-\mathrm{OCH}_{3}\right), 3.43\left(\mathrm{~s}, 3 \mathrm{H},-\mathrm{OCH}_{3}\right), 2.03(\mathrm{~s}, 1 \mathrm{H}$, $-\mathrm{OH}), 1.43-1.31\left(\mathrm{~s}, 24 \mathrm{H}, 4 \mathrm{x}-\mathrm{C}\left(\mathrm{CH}_{3}\right)_{2}\right) ;{ }^{13} \mathrm{C} \mathrm{NMR}$ for first isomer $\left(126 \mathrm{MHz}, \mathrm{CDCl}_{3}\right): \delta$ 137.51 (C-2), 115.89 (C-1), 109.92, $109.77\left(-\mathrm{C}\left(\mathrm{CH}_{3}\right)_{2}\right), 97.18\left(-\mathrm{OCH}_{2} \mathrm{O}-\right), 80.24,79.33$, 77.59, 77.06 (C-4, C-5, C-6, C-7), 73.17 (C-3), 67.99 (C-8), $56.23\left(-\mathrm{OCH}_{3}\right), 27.18,26.66$, 26.42, $25.11\left(-\mathrm{C}\left(\mathrm{CH}_{3}\right)_{2}\right) ;{ }^{13} \mathrm{C} \mathrm{NMR}$ for second isomer $\left(126 \mathrm{MHz}, \mathrm{CDCl}_{3}\right): \delta 136.61(\mathrm{C}-2)$, 117.07 (C-1), 109.79, $109.46\left(-\mathrm{C}\left(\mathrm{CH}_{3}\right)_{2}\right), 98.51$ (-OCH $\left.2 \mathrm{O}-\right), 82.82,80.79,77.45,77.12$ (C-4, 


\section{Supporting information - Experimental Section}

C-5, C-6, C-7), 73.21 (C-3), 68.03 (C-8), $56.21\left(-\mathrm{OCH}_{3}\right), 26.93,26.72,26.40,25.11$ ($\left.\mathrm{C}\left(\mathrm{CH}_{3}\right)_{2}\right)$; FTIR 3466, 2987, 2936, 2886, 1456, 1371, 1212, 1150, 1063, 1027, 919, $845 \mathrm{~cm}^{-1}$; HRMS (ESI) $m / z$ calc for $\mathrm{C}_{16} \mathrm{H}_{28} \mathrm{O}_{7} \mathrm{Na}: 355.1733$, found: $355.1746\left[\mathrm{M}+\mathrm{Na}^{+}\right]$. This mixture of isomers (5.5 g, $16.56 \mathrm{mmol})$ was dissolved in $\mathrm{CH}_{2} \mathrm{Cl}_{2}(80 \mathrm{~mL})$ and the mixture was cooled to $-78{ }^{\circ} \mathrm{C}$. Triethylamine $(2.77 \mathrm{~mL}, 19.9 \mathrm{mmol})$ and $\mathrm{MsCl}(1.54 \mathrm{~mL}, 19.9 \mathrm{mmol})$ were then added slowly and the mixture was stirred for $3 \mathrm{~h}$ at $-78{ }^{\circ} \mathrm{C}$. Upon completion (TLC 1:4 EtOAc: Hexane, $\left.\mathrm{R}_{\mathrm{f}} 0.3\right)$, the mixture was diluted with $\mathrm{CH}_{2} \mathrm{Cl}_{2}(50 \mathrm{~mL})$, washed with sat $\mathrm{NaHCO}_{3}(40 \mathrm{~mL})$ and brine $(40 \mathrm{~mL})$, dried over $\mathrm{Na}_{2} \mathrm{SO}_{4}$, filtered and the solvent was removed under reduced pressure to give a mixture of isomeric mesylates $(\sim 1: 1)$ as a colourless oil (4.1 g, $61 \%)$; ${ }^{1} \mathrm{H}$ NMR (500 MHz, $\left.\mathrm{CDCl}_{3}\right) \delta 6.06-5.95$ (m, 2H, =CH), 5.59 $\left(\mathrm{dd}, J=17.3,10.0 \mathrm{~Hz}, 2 \mathrm{H},=\mathrm{CH}_{2}\right), 5.46\left(\mathrm{dd}, J=15.2,10.5 \mathrm{~Hz}, 2 \mathrm{H},=\mathrm{CH}_{2}\right), 5.32-5.29(\mathrm{~m}$, $1 \mathrm{H},-\mathrm{CH}), 5.21(\mathrm{t}, J=8.0 \mathrm{~Hz}, 1 \mathrm{H},-\mathrm{CH}), 4.80\left(\right.$ apt t $\left., J=7.5 \mathrm{~Hz}, 2 \mathrm{H},-\mathrm{OCH}_{2} \mathrm{O}-\right), 4.76(\mathrm{~d}, J=$ $\left.6.8 \mathrm{~Hz}, 1 \mathrm{H},-\mathrm{OCH}_{2} \mathrm{O}-\right), 4.72\left(\mathrm{~d}, J=6.8 \mathrm{~Hz}, 1 \mathrm{H},-\mathrm{OCH}_{2} \mathrm{O}-\right), 4.18-3.89\left(\mathrm{~m}, 11 \mathrm{H},-\mathrm{CH},-\mathrm{CH}_{2}\right.$, overlapping signals), 3.76 (dd, $J=8.1,1.5 \mathrm{~Hz}, 1 \mathrm{H},-\mathrm{CH}), 3.44\left(\mathrm{~s}, 3 \mathrm{H},-\mathrm{OCH}_{3}\right), 3.42(\mathrm{~s}, 3 \mathrm{H}$, $\left.\mathrm{OCH}_{3}\right), 3.05\left(\mathrm{~s}, 3 \mathrm{H},-\mathrm{SCH}_{3}\right), 3.02\left(\mathrm{~s}, 3 \mathrm{H},-\mathrm{SCH}_{3}\right), 1.43-1.33\left(5 \mathrm{x} \mathrm{s}, 24 \mathrm{H},-\mathrm{C}\left(\mathrm{CH}_{3}\right)_{2}\right) ;{ }^{13} \mathrm{C}$ NMR for the first isomer $\left(126 \mathrm{MHz}, \mathrm{CDCl}_{3}\right) \delta 132.25(\mathrm{C}-2), 121.16$ (C-1), $109.94(-$ $\left.\mathrm{C}\left(\mathrm{CH}_{3}\right)_{2}\right), 97.77$ (-OCH$\left.{ }_{2} \mathrm{O}-\right), 81.93$ (C-3), 79.41, 78.17, 77.10 (C-4, C-5, C-6, C-7), 68.14 (C8), $56.35\left(-\mathrm{OCH}_{3}\right), 39.06\left(-\mathrm{SCH}_{3}\right), 27.14,26.78,26.28,25.22\left(-\mathrm{C}\left(\mathrm{CH}_{3}\right)_{2}\right) ;{ }^{13} \mathrm{C} \mathrm{NMR}$ for the second isomer (126 MHz, $\left.\mathrm{CDCl}_{3}\right) ; \delta 132.01(\mathrm{C}-2), 122.10(\mathrm{C}-1), 109.79,109.78\left(-\mathrm{C}\left(\mathrm{CH}_{3}\right)_{2}\right)$, 98.62 (- $\left.\mathrm{OCH}_{2} \mathrm{O}-\right), 84.62$ (C-3), 78.83, 78.11, 77.35, 77.13 (C-4, C-5, C-6, C-7), 67.90 (C-8), $56.49\left(-\mathrm{OCH}_{3}\right), 39.17\left(-\mathrm{SCH}_{3}\right), 27.17,26.70,26.24,25.11\left(-\mathrm{C}\left(\mathrm{CH}_{3}\right)_{2}\right)$; FTIR 2988, 2937, 2901, 1457, 1415, 1359, 1250, 1213, 1175, 1152, 1065, 1024, 907, 842, 768, 736, $683 \mathrm{~cm}^{-1}$; HRMS (ESI) $m / z$ calc for $\mathrm{C}_{17} \mathrm{H}_{30} \mathrm{O}_{9} \mathrm{SNa}$ : 433.1508, found: 433.1500 [M+Na $\left.{ }^{+}\right]$. This mixture of mesylates (4.1 g, $10.04 \mathrm{mmol})$ was dissolved in anhydrous DMF (50 mL) and $\mathrm{NaN}_{3}(0.9 \mathrm{~g}$, $13.06 \mathrm{mmol}$ ) was added. The mixture was heated to $100{ }^{\circ} \mathrm{C}$ for $3 \mathrm{~h}$. The mixture was cooled to room temp, diluted with EtOAc $(50 \mathrm{~mL})$ and washed with $\mathrm{H}_{2} \mathrm{O}(20 \mathrm{~mL})$. The aq layer was extracted with EtOAc $(3 \times 20 \mathrm{~mL})$, dried over $\mathrm{Na}_{2} \mathrm{SO}_{4}$ and the solvent was removed to give a

yellow syrup. Chromatography of the residue (1:6 EtOAc-hexane) gave azide $\mathbf{S 3}$ as a colourless oil (2.40 g, $67 \%) ; \mathrm{R}_{\mathrm{f}}$ by TLC, 0.5, 1:4 EtOAc-hexane; $[\alpha]_{\mathrm{D}}+67\left(c 1.7, \mathrm{CHCl}_{3}\right) ;{ }^{1} \mathrm{H}$ NMR (500 MHz, $\left.\mathrm{CDCl}_{3}\right) \delta 5.87-5.77$ (m, 2H, C-2, C-3, overlapping signals), 4.74 (d, $J=$ $\left.6.8 \mathrm{~Hz}, 1 \mathrm{H},-\mathrm{OCH}_{2} \mathrm{O}-\right), 4.62$ (d, $\left.J=6.8 \mathrm{~Hz}, 1 \mathrm{H},-\mathrm{OCH}_{2} \mathrm{O}-\right), 4.23$ (dd, $J=7.0,3.7 \mathrm{~Hz}, 1 \mathrm{H}, \mathrm{H}-$ 4), 4.14 (dd, $J=8.2,6.1 \mathrm{~Hz}, 1 \mathrm{H}, \mathrm{H}-8$ ), 4.10 - 3.98 (m, 3H, H-5, H-6, H-7, overlapping signals), 3.94 (dd, $J=8.4,5.5 \mathrm{~Hz}, 1 \mathrm{H}, \mathrm{H}-8$ '), 3.86 - 3.78 (m, 2H, H-1, H-1'), 3.40 (s, 3H, $\left.\mathrm{OCH}_{3}\right), 1.44,1.42,1.39,1.35\left(\mathrm{~s}, 12 \mathrm{H}, 2 \mathrm{x}-\mathrm{C}\left(\mathrm{CH}_{3}\right)_{2}\right) ;{ }^{13} \mathrm{C} \mathrm{NMR}\left(126 \mathrm{MHz}, \mathrm{CDCl}_{3}\right) \delta 131.8$ 


\section{Supporting information - Experimental Section}

(C-2), $128.0(\mathrm{C}-3), 110.0\left(-\mathrm{C}\left(\mathrm{CH}_{3}\right)_{2}\right), 109.6\left(-\mathrm{C}\left(\mathrm{CH}_{3}\right)_{2}\right), 94.0\left(-\mathrm{OCH}_{2} \mathrm{O}\right), 82.4,77.5,77.1(\mathrm{C}-$ 5, C-6, C-7), 75.4 (C-4), 67.4 (C-8), $55.8\left(-\mathrm{OCH}_{3}\right), 52.1$ (C-1), 27.4, 27.0, 26.4, 25.2 ($\left.\mathrm{C}\left(\mathrm{CH}_{3}\right)_{2}\right)$; FTIR 2988, 2935, 2886, $2100\left(-\mathrm{N}_{3}\right), 1456,1381,1371,1212,1151,1064,1022$, 979, 919, $845 \mathrm{~cm}^{-1}$; HRMS (ESI) $\mathrm{m} / z$ calc for $\mathrm{C}_{16} \mathrm{H}_{27} \mathrm{O}_{6} \mathrm{~N}_{3} \mathrm{Na}$ : 380.1798, found: 380.1811 $[\mathrm{M}+\mathrm{Na}]^{+}$.

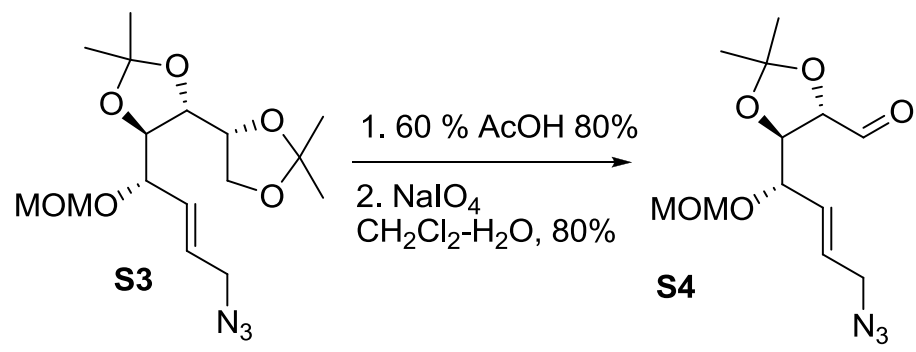

Compound S4 The allylic azide S3 (2.4 g, $6.72 \mathrm{mmol})$ was suspended in $60 \% \mathrm{AcOH}$ $(40 \mathrm{~mL})$ and stirred at room temp for $12 \mathrm{~h}$. The mixture was the solvent was removed and flash column chromatography (1:1 EtOAc-hexane) gave the intermediate diol as a clear oil (1.6 g, $73 \%)$; Rf by TLC, 0.1 (1:1 EtOAc-hexane); $[\alpha]_{\mathrm{D}}+66\left(c\right.$ 1.6, $\left.\mathrm{CHCl}_{3}\right) ;{ }^{1} \mathrm{H}^{\mathrm{NMR}}(500$ $\left.\mathrm{MHz}, \mathrm{CDCl}_{3}\right) \delta 5.88-5.77$ (m, 2H, H-2, H-3, overlapping signals), 4.75 (d, $J=6.8 \mathrm{~Hz}, 1 \mathrm{H}$, $\left.\mathrm{OCH}_{2} \mathrm{O}-\right), 4.65$ (d, $\left.J=6.6 \mathrm{~Hz}, 1 \mathrm{H},-\mathrm{OCH}_{2} \mathrm{O}-\right), 4.43$ (dd, $\left.J=6.7,4.3 \mathrm{~Hz}, 1 \mathrm{H}, \mathrm{H}-4\right), 4.08$ (dd, $J$ = 7.5, 4.2 Hz, 1H, H-5), 3.98 (t, $J=7.4 \mathrm{~Hz}, 1 \mathrm{H}, \mathrm{H}-6), 3.85-3.79$ (m, 3H, H-1, H-1', H-8, overlapping signals), $3.74-3.69$ (m, 2H, H-7, H-8', overlapping signals), 3.41 (s, 3H, $\left.\mathrm{OCH}_{3}\right), 1.40\left(\mathrm{~s}, 6 \mathrm{H},-\mathrm{C}\left(\mathrm{CH}_{3}\right)_{2}\right) ;{ }^{13} \mathrm{C}$ NMR $\left(126 \mathrm{MHz}, \mathrm{CDCl}_{3}\right) \delta 130.3$ (C-3), 128.5 (C-2), $109.6\left(-\mathrm{C}\left(\mathrm{CH}_{3}\right)_{2}\right), 94.5$ (-OCH $\left.2 \mathrm{O}-\right), 81.1$ (C-5), 77.0 (C-6), 75.1 (C-4), 72.7 (C-7), 63.9 (C-8), $56.0\left(-\mathrm{OCH}_{3}\right), 52.1(\mathrm{C}-1), 27.1\left(-\mathrm{C}\left(\mathrm{CH}_{3}\right)_{2}\right), 26.9\left(-\mathrm{C}\left(\mathrm{CH}_{3}\right)_{2}\right)$; FTIR 3423, 2988, 2935, 2891, $2100\left(-\mathrm{N}_{3}\right), 1456,1371,1237,1213,1151,1068,1022,980,919,864 \mathrm{~cm}^{-1}$; HRMS (ESI) $\mathrm{m} / z$ calc for $\mathrm{C}_{13} \mathrm{H}_{23} \mathrm{O}_{6} \mathrm{~N}_{3} \mathrm{Na}$ : 340.1509, found: $340.1498[\mathrm{M}+\mathrm{Na}]^{+}$. This diol (1.6 g, $\left.4.92 \mathrm{mmol}\right)$ was dissolved in $\mathrm{CH}_{2} \mathrm{Cl}_{2}-\mathrm{H}_{2} \mathrm{O}(1: 1,40 \mathrm{~mL})$ and cooled to $0{ }^{\circ} \mathrm{C} . \mathrm{NaIO}_{4}(1.4 \mathrm{~g}, 6.39 \mathrm{mmol})$ was then added and the mixture was allowed to attain room temp. The mixture was diluted with $\mathrm{H}_{2} \mathrm{O}(20 \mathrm{~mL})$ and the layers separated. The aq layer was extracted with $\mathrm{CH}_{2} \mathrm{Cl}_{2}(3 \times 20$ $\mathrm{mL}$ ) and the combined organic layers were dried over $\mathrm{Na}_{2} \mathrm{SO}_{4}$, filtered and the solvent was removed under reduced pressure to give $\mathbf{S} 4$ as a clear oil (1.1 g, $79 \%$ ); (Rf by TLC 0.35, 1:1 EtOAc-hexane); $[\alpha]_{\mathrm{D}}+37\left(c\right.$ 1.6, $\left.\mathrm{CHCl}_{3}\right) ;{ }^{1} \mathrm{H} \mathrm{NMR}\left(500 \mathrm{MHz}, \mathrm{CDCl}_{3}\right) \delta 9.69(\mathrm{~d}, J=1.4 \mathrm{~Hz}$, $1 \mathrm{H}, \mathrm{H}-1), 5.82$ (dt, $J=15.5,6.0 \mathrm{~Hz}, 1 \mathrm{H}, \mathrm{H}-2), 5.67$ (dd, $J=15.6,7.4 \mathrm{~Hz}, 1 \mathrm{H}, \mathrm{H}-3), 4.66$ (d, $J$ $\left.=6.6 \mathrm{~Hz}, 1 \mathrm{H},-\mathrm{OCH}_{2} \mathrm{O}-\right), 4.57\left(\mathrm{~d}, J=6.8 \mathrm{~Hz}, 1 \mathrm{H},-\mathrm{OCH}_{2} \mathrm{O}-\right), 4.24-4.21(\mathrm{~m}, 2 \mathrm{H}, \mathrm{H}-4, \mathrm{H}-6$, overlapping signals), 4.17 (dd, $J=6.6,5.3 \mathrm{~Hz}, 1 \mathrm{H}, \mathrm{H}-5), 3.80-3.73$ (m, 2H, H-1, H-1'), 


\section{Supporting information - Experimental Section}

$3.33\left(\mathrm{~s}, 3 \mathrm{H},-\mathrm{OCH}_{3}\right), 1.44,1.33\left(\mathrm{~s}, 6 \mathrm{H},-\mathrm{C}\left(\mathrm{CH}_{3}\right)_{2}\right) ;{ }^{13} \mathrm{C} \mathrm{NMR}\left(126 \mathrm{MHz}, \mathrm{CDCl}_{3}\right) \delta 199.7(\mathrm{C}-$ 1), 129.0 (C-3), 128.2 (C-2), 110.9 (-C( $\left.\left.\mathrm{CH}_{3}\right)_{2}\right), 93.1$ (-OCH $\left.2 \mathrm{O}-\right), 80.3$ (C-6), 77.5 (C-5), 74.4 (C-4), $54.7\left(-\mathrm{OCH}_{3}\right), 51.0(\mathrm{C}-1), 25.6,25.2\left(-\mathrm{C}\left(\mathrm{CH}_{3}\right)_{2}\right)$; FTIR 2989, 2937, 2897, $2099\left(-\mathrm{N}_{3}\right)$, $1734(\mathrm{C}=\mathrm{O}), 1456,1383,1373,1255,1212,1152,1071,1023,979,918,858,736,702 \mathrm{~cm}^{-1}$; HRMS (ESI) $m / z$ calc for $\mathrm{C}_{12} \mathrm{H}_{23} \mathrm{O}_{5} \mathrm{~N}_{4}: 303.1668$, found: $303.1661\left[\mathrm{M}+\mathrm{NH}_{4}\right]^{+}$.

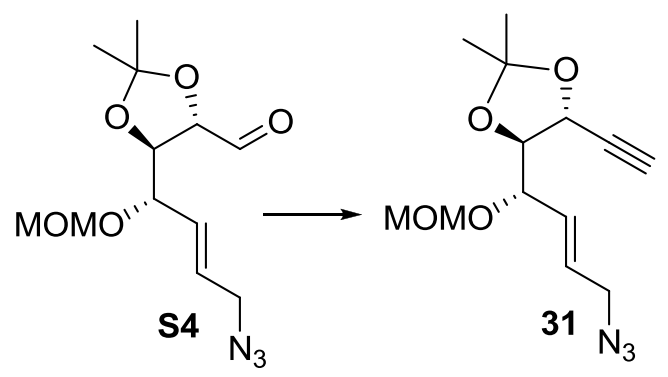

Compound 31 Aldehyde S4 (162 mg, $0.57 \mathrm{mmol})$ was dissolved in $\mathrm{MeOH}(20 \mathrm{~mL})$. $\mathrm{K}_{2} \mathrm{CO}_{3}(23.6 \mathrm{mg}, 1.70 \mathrm{mmol})$ and the Ohira-Bestmann reagent $(0.12 \mathrm{~mL}, 0.74 \mathrm{mmol}$, prepared in situ) were added. The mixture was stirred at room temp overnight. The solvent was removed under diminished pressure. The crude product was re-dissolved in $\mathrm{CH}_{2} \mathrm{Cl}_{2}(20$ $\mathrm{mL})$, washed with $\mathrm{H}_{2} \mathrm{O}(10 \mathrm{~mL})$, dried over $\mathrm{Na}_{2} \mathrm{SO}_{4}$ and the solvent was removed under reduced pressure. Flash column chromatography of the residue (1:4 EtOAc-hexane, $\mathbf{R}_{\mathrm{f}} 0.8$ ) gave 31 as a clear oil $(125 \mathrm{mg}, 78 \%)$. Analytical data for 31: $[\alpha]_{\mathrm{D}}+98.6\left(c 0.98, \mathrm{CHCl}_{3}\right) ;{ }^{1} \mathrm{H}$ $\operatorname{NMR}\left(500 \mathrm{MHz}, \mathrm{CDCl}_{3}\right) \delta 5.89(\mathrm{dt}, J=15.5,6.0 \mathrm{~Hz}, 1 \mathrm{H}, \mathrm{H}-2), 5.75(\mathrm{ddt}, J=15.4,7.3,1.4$ $\mathrm{Hz}, 1 \mathrm{H}, \mathrm{H}-3), 4.73$ (d, $\left.J=6.8 \mathrm{~Hz}, 1 \mathrm{H},-\mathrm{OCH}_{2} \mathrm{O}-\right), 4.64$ (d, $\left.J=6.8 \mathrm{~Hz}, 1 \mathrm{H},-\mathrm{OCH}_{2} \mathrm{O}-\right), 4.52$ (dd, $J=6.8,2.2 \mathrm{~Hz}, 1 \mathrm{H}, \mathrm{H}-6), 4.23$ (dd, $J=7.2,5.8 \mathrm{~Hz}, 1 \mathrm{H}, \mathrm{H}-4), 4.20$ (dd, $J=6.9,5.6 \mathrm{~Hz}$, 1H, H-5), 3.83 (apt t, $J=4.8 \mathrm{~Hz}, 1 \mathrm{H}, \mathrm{H}-1, \mathrm{H}-1$ ') 3.40 (s, 3H, $-\mathrm{OCH}_{3}$ ), 2.54 (d, $J=2.1 \mathrm{~Hz}$, $1 \mathrm{H}, \mathrm{H}-8), 1.51,1.44$ (s, 3H, $\left.-\mathrm{C}\left(\mathrm{CH}_{3}\right)_{2}\right) ;{ }^{13} \mathrm{C} \mathrm{NMR}\left(126 \mathrm{MHz}, \mathrm{CDCl}_{3}\right) \delta 130.1(\mathrm{C}-3), 129.0(\mathrm{C}-$ 2), $111.3\left(-\mathrm{C}\left(\mathrm{CH}_{3}\right)_{2}\right), 94.0$ (-OCH$\left.{ }_{2} \mathrm{O}-\right), 83.2$ (C-5), 81.2 (C-7), 75.1 (C-4), 74.8 (C-8), 66.8 (C-6), $55.6\left(-\mathrm{OCH}_{3}\right), 52.1(\mathrm{C}-1), 26.7,26.0\left(-\mathrm{C}\left(\mathrm{CH}_{3}\right)_{2}\right)$; FTIR 3419, 2988, 2936, $2100\left(-\mathrm{N}_{3}\right)$, 1747, 1371, 1213, 1068, 1025, 980, 917, $879 \mathrm{~cm}^{-1}$; HRMS (ESI) $\mathrm{m} / z$ calc for $\mathrm{C}_{13} \mathrm{H}_{20} \mathrm{~N}_{3} \mathrm{O}_{4}$ : 282.1454: found $282.1445[\mathrm{M}+\mathrm{H}]^{+}$.

\section{Synthetic route to 24}




\section{Supporting information - Experimental Section}

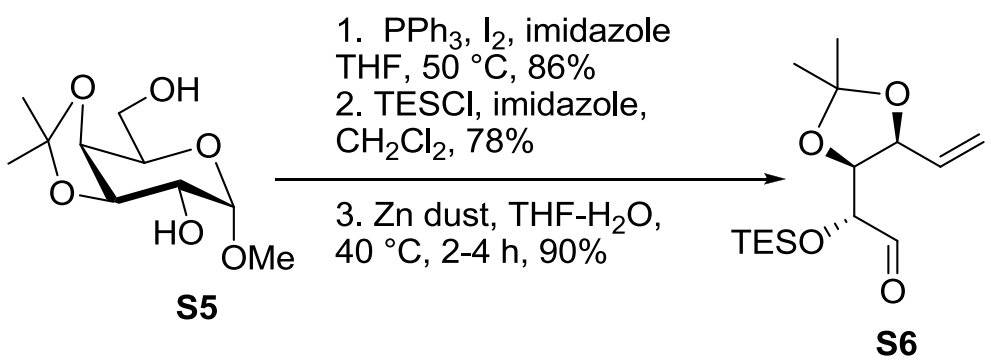

Compound S6. Compound S5 was prepared from methyl $\alpha$-D-galactopyranoside $(6.0 \mathrm{~g}$, $0.031 \mathrm{~mol})$, which was suspended in acetone $(100 \mathrm{~mL})$. 2,2-Dimethoxypropane $(9.5 \mathrm{~mL}$, $0.077 \mathrm{~mol})$ and CSA $(0.4 \mathrm{~g}, 1.54 \mathrm{mmol})$ were charged. The mixture became clear after 10 min and the solution was stirred at room temp for $1.5 \mathrm{~h}$. The reaction was quenched with $\mathrm{NEt}_{3}$ and the solvent was removed under reduced pressure. Flash column chromatography (10 \% MeOH: EtOAc) gave S5 (5.0 g, $70 \%$ ) as a white solid and a minor product, the 4,6-Oisopropylidene protected sugar $(0.7 \mathrm{~g}, 10 \%)$ was also isolated as a white solid. Isopropylidene S5 (2.6 g, $0.01 \mathrm{~mol}$ ), triphenyl phosphine (3.9 g, $0.015 \mathrm{~mol}$ ) and imidazole $(1.4 \mathrm{~g}, 0.02 \mathrm{~mol})$ were dissolved in THF $(100 \mathrm{~mL})$ and the mixture was heated to $50{ }^{\circ} \mathrm{C}$. A solution of iodine $(3.8 \mathrm{~g}, 0.015 \mathrm{~mol})$ in THF $(20 \mathrm{~mL})$ was added portion by portion. The reaction mixture was stirred at reflux for $4 \mathrm{~h}$ and was cooled to room temp and quenched with $10 \%$ aq $\mathrm{Na}_{2} \mathrm{~S}_{2} \mathrm{O}_{3}$. The product was extracted with EtOAc $(3 \times 50 \mathrm{~mL})$, the combined organic layers were washed with brine $(50 \mathrm{~mL})$, dried over $\mathrm{Na}_{2} \mathrm{SO}_{4}$ and the solvent was removed under reduced pressure. Flash column chromatography of the residue (1:10 EtOAc-hexane) gave the 6-deoxy-6-iodo derivative as a pale yellow solid $(3.28 \mathrm{~g}, 86 \%)$; $[\alpha]_{\mathrm{D}}+105.0^{\circ}(c 2.4$, $\left.\mathrm{CHCl}_{3}\right) ;{ }^{1} \mathrm{H} \mathrm{NMR}\left(500 \mathrm{MHz}, \mathrm{CDCl}_{3}\right) \delta 4.68(\mathrm{~d}, J=3.9 \mathrm{~Hz}, 1 \mathrm{H}, \mathrm{H}-1), 4.25(\mathrm{dd}, J=6.2,2.2$ $\mathrm{Hz}, 1 \mathrm{H}, \mathrm{H}-4), 4.21$ (t, $J=6.1 \mathrm{~Hz}, 1 \mathrm{H}, \mathrm{H}-3), 4.05$ (ddd, $J=9.0,6.2,2.7 \mathrm{~Hz}, 1 \mathrm{H}, \mathrm{H}-5), 3.77$ (q, $J=4.3 \mathrm{~Hz}, 1 \mathrm{H}, \mathrm{H}-2), 3.44\left(\mathrm{~s}, 3 \mathrm{H},-\mathrm{OCH}_{3}\right), 3.32$ - 3.19 (m, 2H, H-6, H-6', overlapping signals), $2.86(\mathrm{~s}, 1 \mathrm{H},-\mathrm{OH}), 1.41\left(\mathrm{~s}, 3 \mathrm{H},-\mathrm{C}\left(\mathrm{CH}_{3}\right)_{2}\right), 1.28\left(\mathrm{~s}, 3 \mathrm{H},-\mathrm{C}\left(\mathrm{CH}_{3}\right)_{2}\right) ;{ }^{13} \mathrm{C} \mathrm{NMR}(126$ $\left.\mathrm{MHz}, \mathrm{CDCl}_{3}\right) \delta 109.6\left(-\mathrm{C}\left(\mathrm{CH}_{3}\right)_{2}\right), 98.4$ (C-1), 75.8 (C-3), 73.5 (C-4), 69.2 (C-5), $68.6(\mathrm{C}-2)$, $55.5\left(-\mathrm{OCH}_{3}\right), 27.4\left(-\mathrm{C}\left(\mathrm{CH}_{3}\right)_{2}\right), 25.7\left(-\mathrm{C}\left(\mathrm{CH}_{3}\right)_{2}\right), 2.8$ (C-6); FTIR 3461, 2986, 2934, 2906, 2837, 1453, 1418, 1371, 1339, 1243, 1217, 1146, 1128, 1039, 1002, 904, 886, 865, 831, 797 , 731, $696 \mathrm{~cm}^{-1}$; HRMS (ESI) $\mathrm{m} / z$ calc for $\mathrm{C}_{11} \mathrm{H}_{18} \mathrm{O}_{7} \mathrm{I}$ : 389.0097 Found: $389.0101\left[\mathrm{M}+\mathrm{HCO}_{2}\right]^{-}$. The iodo derivative $(3.5 \mathrm{~g}, 10.29 \mathrm{mmol})$ was dissolved in $\mathrm{CH}_{2} \mathrm{Cl}_{2}(50 \mathrm{~mL})$. Chlorotriethylsilane $(2.1 \mathrm{~mL}, 12.35 \mathrm{mmol})$ and imidazole $(1.8 \mathrm{~g}, 12.35 \mathrm{mmol})$ were charged and the reaction mixture was stirred at room temp for $10 \mathrm{~h}$. The reaction was quenched by addition of $\mathrm{H}_{2} \mathrm{O}(30 \mathrm{~mL})$. The layers were separated and the organic layer washed with $\mathrm{H}_{2} \mathrm{O}$ ( 3 x $20 \mathrm{~mL}$ ), dried over $\mathrm{Na}_{2} \mathrm{SO}_{4}$ and the solvent was removed under reduced pressure. The 


\section{Supporting information - Experimental Section}

crude residue was purified by flash column chromatography (hexane followed by 1:10 EtOAc-hexane) to give TES derivative $(3.7 \mathrm{~g}, 78 \%)$ as a white solid; (1:4 EtOAc-hexane, $\mathrm{R}_{\mathrm{f}}$ 0.8); $[\alpha]_{\mathrm{D}}+92\left(c 1.7, \mathrm{CHCl}_{3}\right) ;{ }^{1} \mathrm{H} \mathrm{NMR}\left(500 \mathrm{MHz}, \mathrm{CDCl}_{3}\right) \delta 4.59(\mathrm{~d}, J=3.6 \mathrm{~Hz}, 1 \mathrm{H}, \mathrm{H}-1)$, $4.28(\mathrm{dd}, J=5.7,2.5 \mathrm{~Hz}, 1 \mathrm{H}, \mathrm{H}-4), 4.15$ (dd, $J=7.3,5.7 \mathrm{~Hz}, 1 \mathrm{H}, \mathrm{H}-3), 4.11$ (ddd, $J=8.3$, 5.7, $2.5 \mathrm{~Hz}, 1 \mathrm{H}, \mathrm{H}-5), 3.71$ (dd, $J=7.3,3.6 \mathrm{~Hz}, 1 \mathrm{H}, \mathrm{H}-2), 3.44$ (s, 3H, - $\left.\mathrm{OCH}_{3}\right), 3.39-3.32$ (m, 2H, H-6, H-6'), 1.48 (s, 3H, -C( $\left.\left.\mathrm{CH}_{3}\right)_{2}\right), 1.33$ (s, 3H, -C( $\left.\left.\mathrm{CH}_{3}\right)_{2}\right), 0.94$ (dt, J = 17.1, 8.0 Hz, $\left.9 \mathrm{H}, \mathrm{Si}\left(\mathrm{CH}_{2} \mathrm{CH}_{3}\right)_{3}\right), 0.62\left(\mathrm{qd}, J=7.9,3.5 \mathrm{~Hz}, 6 \mathrm{H}, \mathrm{Si}\left(\mathrm{CH}_{2} \mathrm{CH}_{3}\right)_{3}\right) ;{ }^{13} \mathrm{C} \mathrm{NMR}\left(126 \mathrm{MHz} \mathrm{CDCl}_{3}\right)$ $\delta 109.1\left(-\mathrm{C}\left(\mathrm{CH}_{3}\right)_{2}\right), 100.2(\mathrm{C}-1), 76.9$ (C-3), 74.0 (C-4), 71.2 (C-2), 68.3 (C-5), 55.7 ($\left.\mathrm{OCH}_{3}\right), 28.1 \quad\left(-\mathrm{C}\left(\mathrm{CH}_{3}\right)_{2}\right), \quad 26.2 \quad\left(-\mathrm{C}\left(\mathrm{CH}_{3}\right)_{2}\right), \quad 6.6 \quad\left(\mathrm{CH}_{3},-\mathrm{Si}\left(\mathrm{CH}_{2} \mathrm{CH}_{3}\right)_{3}\right), \quad 4.7 \quad\left(\mathrm{CH}_{2}\right.$, $\left.\mathrm{Si}\left(\mathrm{CH}_{2} \mathrm{CH}_{3}\right)_{3}\right), 2.8$ (C-6); FTIR 2953, 2910, 2877, 1626, 1552, 1458, 1380, 1314, 1241, 1208 , 1124, 1039, 1015, 968, 916, 871, 842, 800, $725 \mathrm{~cm}^{-1}$; HRMS (ESI) $\mathrm{m} / z$ calc for $\mathrm{C}_{18} \mathrm{H}_{34} \mathrm{O}_{5} \mathrm{SiINNa}$ : 522.1149, Found: 522.1139 [M+MeCN+Na] ${ }^{+}$. To this TES derivative (5.1 $\mathrm{g}, 11.1 \mathrm{mmol})$ was dissolved in a mixture of THF- $\mathrm{H}_{2} \mathrm{O}(9: 1,100 \mathrm{~mL})$. Pre-activated $\mathrm{Zn}$ dust $(7.3 \mathrm{~g}, 0.11 \mathrm{~mol})$ was charged and the mixture was sonicated at $40{ }^{\circ} \mathrm{C}$ for $2-4 \mathrm{~h}$. The mixture was filtered and washed with $\mathrm{Et}_{2} \mathrm{O}(100 \mathrm{~mL})$. The organic layer was washed with $\mathrm{H}_{2} \mathrm{O}(40$ $\mathrm{mL})$, sat aq $\mathrm{NaHCO}_{3}(40 \mathrm{~mL})$ and brine $(40 \mathrm{~mL})$, dried over $\mathrm{Na}_{2} \mathrm{SO}_{4}$ and the solvent was removed under reduced pressure to give S6 $(3.0 \mathrm{~g}, 90 \%)$ as a clear oil, which was used immediately in the next step due to a risk of epimerization; $[\alpha]_{\mathrm{D}}+31.8^{\circ}\left(c 0.77, \mathrm{CHCl}_{3}\right) ;{ }^{1} \mathrm{H}$ $\operatorname{NMR}\left(500 \mathrm{MHz}, \mathrm{CDCl}_{3}\right) \delta 9.65(\mathrm{~d}, J=0.9 \mathrm{~Hz}, 1 \mathrm{H}, \mathrm{C}-1), 5.92(\mathrm{ddd}, J=17.3,10.4,6.9 \mathrm{~Hz}$, 1H, H-5), 5.35 (dt, $J=17.2,1.4$ Hz, 1H, H-6), 5.22 (dt, $J=10.5,1.4$ Hz, 1H, H-6'), 4.72 (t, $J$ $=6.8 \mathrm{~Hz}, 1 \mathrm{H}, \mathrm{H}-4), 4.32(\mathrm{dd}, J=6.7,5.2 \mathrm{~Hz}, 1 \mathrm{H}, \mathrm{H}-3), 4.07$ (dd, $J=5.2,0.9 \mathrm{~Hz}, 1 \mathrm{H}, \mathrm{H}-2)$, $1.50\left(\mathrm{~s}, 3 \mathrm{H},-\mathrm{C}\left(\mathrm{CH}_{3}\right)_{2}\right), 1.35\left(\mathrm{~s}, 3 \mathrm{H},-\mathrm{C}\left(\mathrm{CH}_{3}\right)_{2}\right), 0.96\left(\mathrm{t}, J=7.9 \mathrm{~Hz}, 9 \mathrm{H},-\mathrm{Si}\left(\mathrm{CH}_{2} \mathrm{CH}_{3}\right)_{3}\right), 0.64$ $\left(\mathrm{q}, J=7.8 \mathrm{~Hz}, 6 \mathrm{H},-\mathrm{Si}\left(\mathrm{CH}_{2} \mathrm{CH}_{3}\right)_{3}\right) ;{ }^{13} \mathrm{C} \mathrm{NMR}\left(126 \mathrm{MHz}, \mathrm{CDCl}_{3}\right) \delta 201.7(\mathrm{C}-1), 133.6(\mathrm{C}-5)$, 118.8 (C-6), $109.2\left(-\mathrm{C}\left(\mathrm{CH}_{3}\right)_{2}\right), 78.9$ (C-3), $78.4(\mathrm{C}-4), 77.2(\mathrm{C}-2), 26.8\left(-\mathrm{C}\left(\mathrm{CH}_{3}\right)_{2}\right), 25.2$ ($\left.\mathrm{C}\left(\mathrm{CH}_{3}\right)_{2}\right), 6.7\left(\mathrm{CH}_{3},-\mathrm{Si}\left(\mathrm{CH}_{2} \mathrm{CH}_{3}\right)_{3}\right), 4.9\left(\mathrm{CH}_{2},-\mathrm{Si}\left(\mathrm{CH}_{2} \mathrm{CH}_{3}\right)_{3}\right)$; FTIR 2955, 2913, 2878, 1736, 1458, 1414, 1370, 1241, 1216, 1149, 1074, 1040, 1005, 928, 872, 789, $724 \mathrm{~cm}^{-1}$; HRMS (ESI) $\mathrm{m} / z$ calc for $\mathrm{C}_{17} \mathrm{H}_{31} \mathrm{O}_{5} \mathrm{SiNNa}$ : 364.1920, Found: $364.1905[\mathrm{M}+\mathrm{MeCN}+\mathrm{Na}]^{+}$. 


\section{Supporting information - Experimental Section}

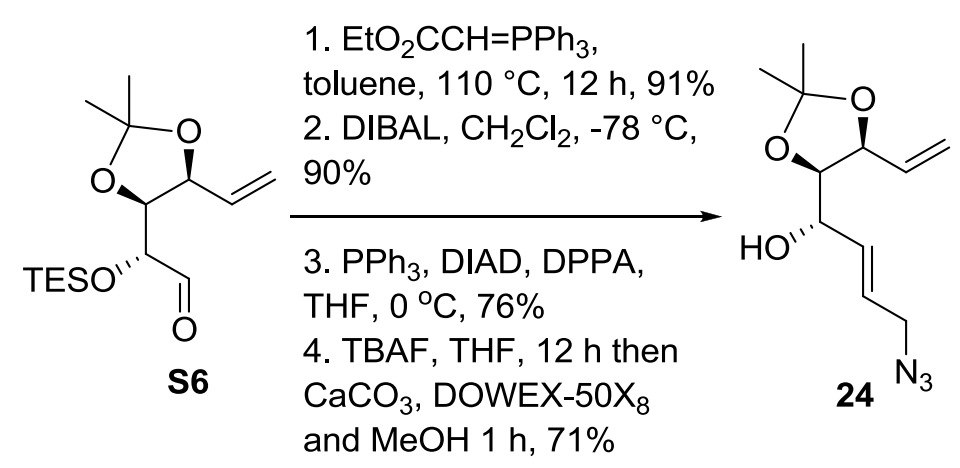

\section{(S,E)-4-Azido-1-((4R,5S)-2,2-dimethyl-5-vinyl-1,3-dioxolan-4-yl)but-2-en-1-ol 24.}

Intermediate S6 (3.4 g, $0.011 \mathrm{~mol})$ was dissolved in toluene $(70 \mathrm{~mL})$ and carbethoxymethylenetriphenylphosphorane $(5.9 \mathrm{~g}, 0.017 \mathrm{~mol})$ was charged and the reaction was heated to reflux for $12 \mathrm{~h}$. The solvent was removed under diminished pressure and flash column chromatography of the residue (1:10 EtOAc-hexane) gave the $E$-alkene product (3.8 g, $91 \%)$ as a clear oil; $[\alpha]_{\mathrm{D}}-4.2\left(c\right.$ 5.1, $\left.\mathrm{CHCl}_{3}\right) ;{ }^{1} \mathrm{H}$ NMR $\left(500 \mathrm{MHz}, \mathrm{CDCl}_{3}\right) \delta 6.96(\mathrm{dd}, J=$ $15.5,4.5 \mathrm{~Hz}, 1 \mathrm{H}, \mathrm{H}-3), 6.10$ (dd, $J=15.6,1.8 \mathrm{~Hz}, 1 \mathrm{H}, \mathrm{H}-2), 5.94$ (ddd, $J=17.1,10.2,8.2$ $\mathrm{Hz}, 1 \mathrm{H}, \mathrm{H}-7$ ), 5.35 (dt, $J=17.1,1.2 \mathrm{~Hz}, 1 \mathrm{H}, \mathrm{H}-8$ ), 5.27 (apt d, $J=10.3, \mathrm{~Hz}, 1 \mathrm{H}, \mathrm{H}-8$ '), 4.50 $(\mathrm{dd}, J=8.1,6.3 \mathrm{~Hz}, 1 \mathrm{H}, \mathrm{H}-6), 4.35$ (ddd $J=7.1,4.5,1.8 \mathrm{~Hz}, 1 \mathrm{H}, \mathrm{H}-4), 4.19$ (q, $J=7.1 \mathrm{~Hz}$, $\left.2 \mathrm{H},-\mathrm{OCH}_{2}\right), 4.01(\mathrm{dd}, J=7.1,6.1 \mathrm{~Hz}, 1 \mathrm{H}, \mathrm{H}-5), 1.51\left(\mathrm{~s}, 3 \mathrm{H},-\mathrm{C}\left(\mathrm{CH}_{3}\right)_{2}\right), 1.35(\mathrm{~d}, 3 \mathrm{H}$, $\left.\mathrm{C}\left(\mathrm{CH}_{3}\right)_{2}\right), 1.29\left(\mathrm{t}, J=7.2 \mathrm{~Hz}, 3 \mathrm{H},-\mathrm{CH}_{3}\right), 0.96\left(\mathrm{t}, J=7.9 \mathrm{~Hz}, 9 \mathrm{H},-\mathrm{Si}\left(\mathrm{CH}_{2} \mathrm{CH}_{3}\right)_{3}\right), 0.62(\mathrm{qd}, J$ = 7.9, $\left.3.5 \mathrm{~Hz}, 6 \mathrm{H},-\mathrm{Si}\left(\mathrm{CH}_{2} \mathrm{CH}_{3}\right)_{3}\right) ;{ }^{13} \mathrm{C} \mathrm{NMR}\left(126 \mathrm{MHz}, \mathrm{CDCl}_{3}\right) \delta 166.3(\mathrm{C}-1), 146.2(\mathrm{C}-3)$, 134.0 (C-7), 122.3 (C-2), 119.2 (C-8), 108.9 (-C( $\left.\left.\mathrm{CH}_{3}\right)_{2}\right), 81.0$ (C-5), 78.9 (C-6), 71.1 (C-4), $60.4\left(-\mathrm{OCH}_{2}\right), 27.6,25.4\left(-\mathrm{C}\left(\mathrm{CH}_{3}\right)_{2}\right), 14.2\left(-\mathrm{CH}_{3}\right), 6.8\left(\mathrm{CH}_{3},-\mathrm{Si}\left(\mathrm{CH}_{2} \mathrm{CH}_{3}\right)_{3}\right), 4.9\left(\mathrm{CH}_{2}\right.$, $\left.\mathrm{Si}\left(\mathrm{CH}_{2} \mathrm{CH}_{3}\right)_{3}\right)$; FTIR 2983, 2956, 2920, 2877, 1722, 1660, 1458, 1413, 1380, 1368, 1279, 1216, 1163, 1131, 1073, 1038, 1003, 931, 839, 796, 739, $727 \mathrm{~cm}^{-1}$; HRMS (ESI) $\mathrm{m} / \mathrm{z}$ calc for $\mathrm{C}_{19} \mathrm{H}_{34} \mathrm{O}_{5} \mathrm{SiNa}$ : 393.2073, Found: $393.2089[\mathrm{M}+\mathrm{Na}]^{+}$. This unsaturated ester $(3.6 \mathrm{~g}, 9.78$ mmol) was dissolved in $\mathrm{CH}_{2} \mathrm{Cl}_{2}(100 \mathrm{~mL})$ and cooled to $-78{ }^{\circ} \mathrm{C}$. DIBAL-H $(30 \mathrm{~mL}, 29.35$ mmol, $1.0 \mathrm{M}$ in $\mathrm{CH}_{2} \mathrm{Cl}_{2}$ ) was added dropwise. The reaction was stirred at $-78{ }^{\circ} \mathrm{C}$ for $4 \mathrm{~h}$. The mixture was quenched with $\mathrm{MeOH}$ at $-78{ }^{\circ} \mathrm{C}$ and warmed to room temp. The mixture was stirred with aq sodium potassium tartrate solution until clear. The layers were separated and the organic layer was washed with $\mathrm{H}_{2} \mathrm{O}(50 \mathrm{~mL})$, dried over $\mathrm{Na}_{2} \mathrm{SO}_{4}$ and the solvent was removed. The residue was purified by column chromatography (1:4 EtOAc-hexane) to give the primary alcohol product $(2.9 \mathrm{~g}, 90 \%)$ as a clear oil (1:4 EtOAc-hexane, $\left.\mathrm{R}_{\mathrm{f}} 0.45\right) ;[\alpha]_{\mathrm{D}}$ $4.5\left(\mathrm{c} 2.7, \mathrm{CHCl}_{3}\right) ;{ }^{1} \mathrm{H}$ NMR $\left(500 \mathrm{MHz}, \mathrm{CDCl}_{3}\right) \delta 5.94(\mathrm{ddd}, J=17.2,10.3,8.1 \mathrm{~Hz}, 1 \mathrm{H}, \mathrm{H}-$ 7), 5.87 (dtd, $J=15.5,5.3,1.3 \mathrm{~Hz}, 1 \mathrm{H}, \mathrm{H}-2), 5.71$ (ddt, $J=15.4,5.8,1.5 \mathrm{~Hz}, 1 \mathrm{H}, \mathrm{H}-3), 5.27$ 


\section{Supporting information - Experimental Section}

(apt d, $J=17.2 \mathrm{~Hz}, 1 \mathrm{H}, \mathrm{H}-8$ ), 5.21 (apt d, $J=10.2 \mathrm{~Hz}, 1 \mathrm{H}, \mathrm{H}-8^{\prime}$ ), $4.46-4.43$ (apt t, $J=7.0$ Hz, 1H, H-6), 4.18 - 4.15 (m, 1H, H-4), 4.10 (d, $J=5.2$ Hz, 2H, H-1, H-1'), 3.97 (t, $J=6.5$ $\mathrm{Hz}, 1 \mathrm{H}, \mathrm{H}-5), 1.47$ (s, 3H, -C( $\left.\left(\mathrm{CH}_{3}\right)_{2}\right), 1.33\left(\mathrm{~s}, 3 \mathrm{H},-\mathrm{C}\left(\mathrm{CH}_{3}\right)_{2}\right), 0.93$ (t, $J=8.0 \mathrm{~Hz}, 9 \mathrm{H}$, $\left.\mathrm{Si}\left(\mathrm{CH}_{2} \mathrm{CH}_{3}\right)_{3}\right), 0.59\left(\mathrm{qd}, J=7.9,3.4 \mathrm{~Hz}, 6 \mathrm{H},-\mathrm{Si}\left(\mathrm{CH}_{2} \mathrm{CH}_{3}\right)_{3}\right) ;{ }^{13} \mathrm{C} \mathrm{NMR}\left(126 \mathrm{MHz}, \mathrm{CDCl}_{3}\right) \delta$ 134.5 (C-7), 131.4 (C-2), 129.8 (C-3), 118.6 (C-8), $108.7\left(-\mathrm{C}\left(\mathrm{CH}_{3}\right)_{2}\right), 81.8$ (C-5), 79.0 (C-6), 71.6 (C-4), $62.8(\mathrm{C}-1), 27.6,25.5\left(-\mathrm{C}\left(\mathrm{CH}_{3}\right)_{2}\right), 6.8\left(\mathrm{CH}_{3},-\mathrm{Si}\left(\mathrm{CH}_{2} \mathrm{CH}_{3}\right)_{3}\right), 5.0\left(\mathrm{CH}_{2}\right.$, $\left.\mathrm{Si}\left(\mathrm{CH}_{2} \mathrm{CH}_{3}\right)_{3}\right)$; FTIR 3391, 2954, 2909, 2877, 1458, 1414, 1379, 1369, 1238, 1217, 1132, 1090, 1061, 1037, 1002, 975, 927, 873, 847, 739, $725 \mathrm{~cm}^{-1}$; HRMS (ESI) $\mathrm{m} / z$ calc for $\mathrm{C}_{17} \mathrm{H}_{32} \mathrm{O}_{4} \mathrm{SiNa}: 351.1968$, Found: $351.1975[\mathrm{M}+\mathrm{Na}]^{+}$. This alcohol (2.5 g, $\left.7.50 \mathrm{mmol}\right)$ was dissolved in THF (50 mL) and triphenylphosphine (3.3 g, $12.74 \mathrm{~mol})$ was charged and the mixture was cooled to $0{ }^{\circ} \mathrm{C}$. Diisopropylazodicarboxylate $(2.50 \mathrm{~mL}, 12.7 \mathrm{mmol})$ and diphenylphosphoryl azide $(2.80 \mathrm{~mL}, 12.7 \mathrm{mmol})$ were added dropwise. The mixture was warmed to room temp and stirred for $8 \mathrm{~h}$. The solvent was removed under diminished pressure and flash column chromatography of the residue (100\% hexane) gave the TES protected allylic azide $(2.0 \mathrm{~g}, 76 \%)$ as a pale yellow oil. (TLC 1:20 EtOAc-hexane, $\mathrm{R}_{\mathrm{f}} 0.8$ ); $[\alpha]_{\mathrm{D}}-4.0\left(c 6.0\right.$ in $\left.\mathrm{CHCl}_{3}\right) ;{ }^{1} \mathrm{H}$ NMR $\left(500 \mathrm{MHz}, \mathrm{CDCl}_{3}\right) \delta 5.95(\mathrm{ddd}, J=17.1,10.3,8.1 \mathrm{~Hz}$, $1 \mathrm{H}, \mathrm{H}-7$ ), $5.86-5.78$ (m, 2H, H-2, H-2, overlapping signals), 5.30 (apt d, $J=17.2 \mathrm{~Hz}, 1 \mathrm{H}$, H-8), 5.25 (apt d, $J=10.1 \mathrm{~Hz}, 1 \mathrm{H}, \mathrm{H}-8$ '), 4.46 (dd, $J=8.2,6.2 \mathrm{~Hz}, 1 \mathrm{H}, \mathrm{H}-6), 4.21$ (dd, $J=$ 6.9, 3.1 Hz, 1H, H-4), 3.98 (t, $J=6.5 \mathrm{~Hz}, 1 \mathrm{H}, \mathrm{H}-5$ ), 3.75 (apt d, $J=4.1 \mathrm{~Hz}, 2 \mathrm{H}, \mathrm{H}-1, \mathrm{H}-1$ ' overlapping signals), 1.49 (s, 3H, - $\left.\mathrm{C}\left(\mathrm{CH}_{3}\right)_{2}\right), 1.35$ (s, 3H, - $\left.\mathrm{C}\left(\mathrm{CH}_{3}\right)_{2}\right), 0.96(\mathrm{t}, J=8.0 \mathrm{~Hz}, 9 \mathrm{H}$, $\left.\mathrm{Si}\left(\mathrm{CH}_{2} \mathrm{CH}_{3}\right)_{3}\right), 0.62\left(\mathrm{qd}, J=7.8,3.8 \mathrm{~Hz}, 6 \mathrm{H},-\mathrm{Si}\left(\mathrm{CH}_{2} \mathrm{CH}_{3}\right)_{3}\right) ;{ }^{13} \mathrm{C} \mathrm{NMR}\left(126 \mathrm{MHz}, \mathrm{CDCl}_{3}\right) \delta$ 134.5 (d, C-7), 134.0 (d, C-2), 125.3 (d, C-3), 118.8 (t, C-8), 108.7 (s, -C( $\left.\left.\mathrm{CH}_{3}\right)_{2}\right), 81.8$ (d, C5), 79.0 (d, C-6), 71.4 (d, C-4), 52.2 (t, C-1), 27.7 (q, - $\left.\mathrm{C}\left(\mathrm{CH}_{3}\right)_{2}\right), 25.5$ (q, - $\left.\mathrm{C}\left(\mathrm{CH}_{3}\right)_{2}\right), 6.8$ (q, $\left.\mathrm{Si}\left(\mathrm{CH}_{2} \mathrm{CH}_{3}\right)_{3}\right), 5.0$ (t, -Si $\left.\left(\mathrm{CH}_{2} \mathrm{CH}_{3}\right)_{3}\right)$; FTIR 2988, 2955, 2913, 2877, $2098\left(-\mathrm{N}_{3}\right), 1458,1414$, 1380, 1370, 1238, 1215, 1133, 1068, 1038, 1003, 975, 930, 871, 848, 739, $725 \mathrm{~cm}^{-1}$; HRMS (ESI) $\mathrm{m} / z$ calc for $\mathrm{C}_{17} \mathrm{H}_{32} \mathrm{O}_{3} \mathrm{~N}_{3} \mathrm{Si}$ : 354.2213, Found: $354.2225[\mathrm{M}+\mathrm{H}]^{+}$. This intermediate $(2.0 \mathrm{~g}, 5.7 \mathrm{mmol})$ was dissolved in THF $70(\mathrm{~mL})$. TBAF (12 mL, $11.3 \mathrm{mmol}, 1.0 \mathrm{M}$ in THF) was added slowly and the reaction was stirred at room temp for $12 \mathrm{~h}$. The solution was then stirred with $\mathrm{CaCO}_{3}$, DOWEX-50X8 and $\mathrm{MeOH}$ for $1 \mathrm{~h}$. The slurry was filtered through a pad of celite and washed thoroughly with $\mathrm{MeOH}$ and the solvent was removed under reduced pressure. Flash column chromatography (1:1 EtOAc-hexane) gave $24(1.0 \mathrm{~g}, 71 \%)$ as a clear oil (1:4 EtOAc-hexane $\left.\mathrm{R}_{\mathrm{f}} 0.3\right) ;[\alpha]_{\mathrm{D}}+6.8\left(c 6.0, \mathrm{CHCl}_{3}\right) ;{ }^{1} \mathrm{H} \mathrm{NMR}\left(500 \mathrm{MHz}, \mathrm{CDCl}_{3}\right) \delta 6.01$ (ddd, $J=17.1,10.3,7.8 \mathrm{~Hz}, 1 \mathrm{H}, \mathrm{H}-7), 5.88$ (dtd, $J=15.5,6.1,1.2 \mathrm{~Hz}, 1 \mathrm{H}, \mathrm{H}-2), 5.78$ (apt dd, $J=15.4,5.6 \mathrm{~Hz}, 1 \mathrm{H}, \mathrm{H}-3), 5.40$ (apt d, $J=17.0 \mathrm{~Hz}, 1 \mathrm{H}, \mathrm{H}-8), 5.33$ (apt d, $J=10.3 \mathrm{~Hz}, 1 \mathrm{H}$, 


\section{Supporting information - Experimental Section}

H-8'), 4.64 (t, $J=7.2 \mathrm{~Hz}, 1 \mathrm{H}, \mathrm{H}-6), 4.18-4.17$ (m, 1H, H-4), 4.08 (dd, $J=6.8,5.5 \mathrm{~Hz}, 1 \mathrm{H}$, H-5), 3.80 (apt d, J = 6.0 Hz, 2H, H-1, H-1'), 2.41 (s, 1H, -OH), 1.55 (s, 3H, -C(CH (CH) $\left._{2}\right), 1.41$ (s, 3H, -C( $\left.\left(\mathrm{CH}_{3}\right)_{2}\right) ;{ }^{13} \mathrm{C}$ NMR (126 MHz, $\left.\mathrm{CDCl}_{3}\right) \delta 133.6$ (C-7), 133.5 (C-3), 126.0 (C-2), 119.7 (C-8), 108.9 (-C(CH$\left.)_{2}\right), 80.6$ (C-5), 78.9 (C-6), 69.7 (C-4), 52.1 (C-1), 27.4, 25.0 ($\left.\mathrm{C}\left(\mathrm{CH}_{3}\right)_{2}\right)$; FTIR 3470, 2987, 2936, 2893, $2097\left(-\mathrm{N}_{3}\right), 1734,1644,1429,1381,1243,1213$,

1164, 1038, 993, 975, 869, 813, $664 \mathrm{~cm}^{-1}$; HRMS (ESI) $\mathrm{m} / z$ calc for $\mathrm{C}_{11} \mathrm{H}_{18} \mathrm{O}_{3} \mathrm{~N}_{3}: 240.1348$, Found: $240.1351[\mathrm{M}+\mathrm{H}]^{+}$. 


\section{Supporting information - Experimental Section}
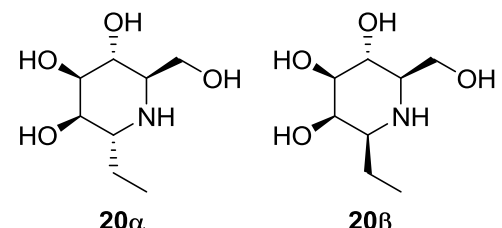

Table S1: ${ }^{1} \mathrm{H}-\mathrm{NMR}$ data for $20 \alpha$ and comparison with natural product

\begin{tabular}{|c|c|c|c|}
\hline Proton & $\begin{array}{l}\delta 20 \alpha \text { prepared in this } \\
\text { manuscript }\left(D_{2} \mathrm{O}, 500 \mathrm{MHz}, \mathrm{J}\right. \\
\text { in } \mathrm{Hz} \text {, ref HOD at } \delta 4.80 \\
\text { ppm) }\end{array}$ & $\begin{array}{l}\text { Natural product } \\
\text { isolated by Kato et al ( } \\
\text { J. Nat. Prod. 2007, 70, } \\
993-997 \text { ) }\end{array}$ & $\begin{array}{l}20 \beta \text { prepared by } \\
\text { Fleet et al } \\
\text { (Tetrahedron Lett. } \\
1989,30,4439-4442 \text { ) }\end{array}$ \\
\hline H-1 & $2.93, \mathrm{td}, \mathrm{J}=7.7,2.4$ & 2.98, not given & \multirow{8}{*}{ Not reported } \\
\hline H-2 & $3.97, \mathrm{t}, \mathrm{J}=2.8$ & $3.98, J=2.5$ & \\
\hline H-3 & $3.76, d d, J=9.5,3.2$ & $3.76, J=9.5,3.1$ & \\
\hline $\mathrm{H}-4$ & $3.64, t, J=9.7$ & $3.66, J=9.5$ & \\
\hline H-5 & $2.74, \mathrm{dt}, \mathrm{J}=4.0,9.9$ & 2.79, not given & \\
\hline H-6a, H-6b & $3.79, d, J=4.0$ & $3.79, \mathrm{~J}=3.5$ & \\
\hline $\mathrm{CH}_{2}$ & $1.59, p, J=7.5$ & 1.60 , not given & \\
\hline $\mathrm{CH}_{3}$ & $0.97, t, J=7.5$ & $0.97, \mathrm{~J}=7.6$ & \\
\hline
\end{tabular}

Table S2: ${ }^{13}$ C-NMR data for $20 \alpha$ and comparison with natural product and $20 \beta$

\begin{tabular}{|c|c|c|c|}
\hline Carbon & $\begin{array}{l}\delta 20 \alpha \text { prepared as described } \\
\text { in this manuscript }\left(D_{2} O, 500\right. \\
M H z \text { and adjusted so that } C- \\
5 \text { are equal }\end{array}$ & $\begin{array}{l}\text { Natural product } \\
\text { isolated by Kato et al } \\
\text { ( J. Nat. Prod. 2007, 70, } \\
993-997 \text { ) }\end{array}$ & $\begin{array}{l}20 \beta \text { prepared by Fleet } \\
\text { et al (Tetrahedron Lett. } \\
1989,30,4439-4442)\end{array}$ \\
\hline C-1 & 62.0 & 62.1 & 60.95 or 59.17 \\
\hline C-2 & 74.1 & 73.1 & 76.21 or 70.42 or 69.14 \\
\hline C-3 & 74.4 & 74.1 & 76.21 or 70.42 or 69.14 \\
\hline C-4 & 71.6 & 71.2 & 76.21 or 70.42 or 69.14 \\
\hline C-5 & 57.8 & 57.8 & 60.95 or 59.17 \\
\hline C-6 & 63.8 & 63.4 & 61.61 \\
\hline $\mathrm{CH}_{2}$ & 24.5 & 24.3 & 24.19 \\
\hline $\mathrm{CH}_{3}$ & 13.5 & 13.4 & 10.66 \\
\hline
\end{tabular}




\section{Supporting information - Experimental Section}

\section{Plot of $\Delta \delta$ (Y axis) against signal \\ number (X-axis)}

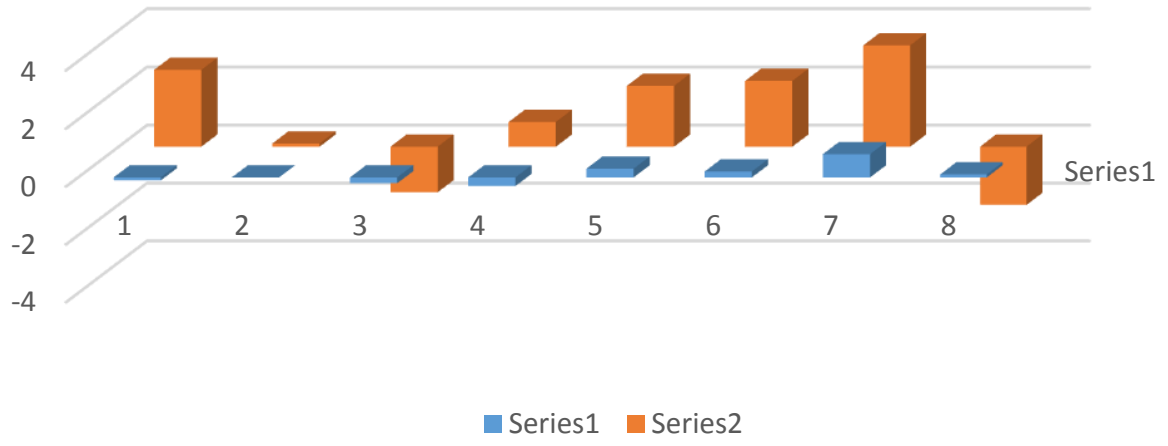

Series 1 (see Table S3) = (Moynihan et al, this manuscript, A) - (Kato et al, B) or column A minus B Series 2 (See Table S3) = (Moynihan et al, this manuscript, A) - (Fleet et al, C) or column A minus C Table S3 Data used in plot above

\begin{tabular}{|l|r|r|r|r|r|}
\hline Signal & \multicolumn{1}{c|}{$\begin{array}{c}\text { Moynihan } \\
\text { et al }\end{array}$} & $\begin{array}{c}\text { Kato et } \\
\text { al }\end{array}$ & $\begin{array}{c}\text { Fleet et } \\
\text { al }\end{array}$ & \multicolumn{1}{c|}{$\begin{array}{c}\Delta \delta \\
\text { C }\end{array}$} & $\begin{array}{c}\Delta \\
\text { A-B }\end{array}$ \\
\hline 1 & 13.5 & 13.4 & 10.66 & 0.1 & \multicolumn{1}{c|}{ A-C } \\
\hline 2 & 24.5 & 24.3 & 24.19 & 0.2 & 0.31 \\
\hline 3 & 57.8 & 57.8 & 59.17 & 0 & -1.37 \\
\hline 4 & 62 & 62.1 & 60.95 & -0.1 & 1.05 \\
\hline 5 & 63.8 & 63.4 & 61.61 & 0.4 & 2.19 \\
\hline 6 & 71.6 & 71.2 & 69.14 & 0.4 & 2.46 \\
\hline 7 & 74.1 & 73.1 & 70.42 & 1 & 3.68 \\
\hline 8 & 74.4 & 74.1 & 76.21 & 0.3 & -1.81 \\
\hline
\end{tabular}




\section{Supporting information - Experimental Section}

${ }^{13}$ C NMR Data used for assignment of stereochemical configuration to 27

\begin{tabular}{|c|c|c|c|}
\hline Signal number & $\begin{array}{l}{ }^{13} \mathrm{C}-\mathrm{NMR} \text { data for } \mathbf{2 7} \text { for ring carbon } \\
\text { atoms }(\delta, \mathrm{ppm}) \text { adjusted to make } \\
\text { signal } 4 \text { equal to the corresponding } \\
\text { signal in } \mathbf{3 0}\end{array}$ & $\begin{array}{c}{ }^{13} \mathrm{C}-\mathrm{NMR} \text { data for } \mathbf{3 0} \\
\text { for ring carbon } \\
\text { atoms }(\delta, \mathrm{ppm}) \\
\text { G }\end{array}$ & $\begin{array}{c}\Delta \delta \\
\text { Columns } \\
\text { F minus G }\end{array}$ \\
\hline
\end{tabular}

Reference for NMR data for $\mathbf{2 9 / 3 0}$ is J. Chem. Soc., Perkin Trans. 1, 1999, 2735-2745

\begin{tabular}{|c|c|c|c|c|}
\hline \multirow{3}{*}{ Signal number } & \multirow{2}{*}{\multicolumn{2}{|c|}{ 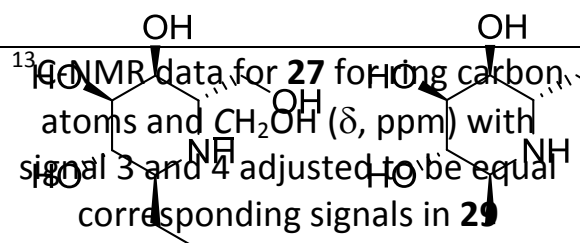 }} & \multicolumn{2}{|l|}{$\mathrm{OH}$} \\
\hline & & & 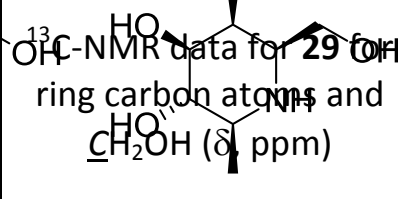 & $\Delta \delta$ \\
\hline & $27 \quad$ D & 29 & 30 & minus $\mathrm{E}$ \\
\hline 1 & 56.7 & & 51.2 & 5.5 \\
\hline 2 & 59.5 & & 59.3 & 0.2 \\
\hline 3 & 59.6 & & 59.6 & 0 \\
\hline 4 & 69.3 & & 69.3 & 0 \\
\hline 5 & 71.7 & & 71.8 & -0.1 \\
\hline 6 & 72.3 & & 73.6 & -1.3 \\
\hline
\end{tabular}

Table S4

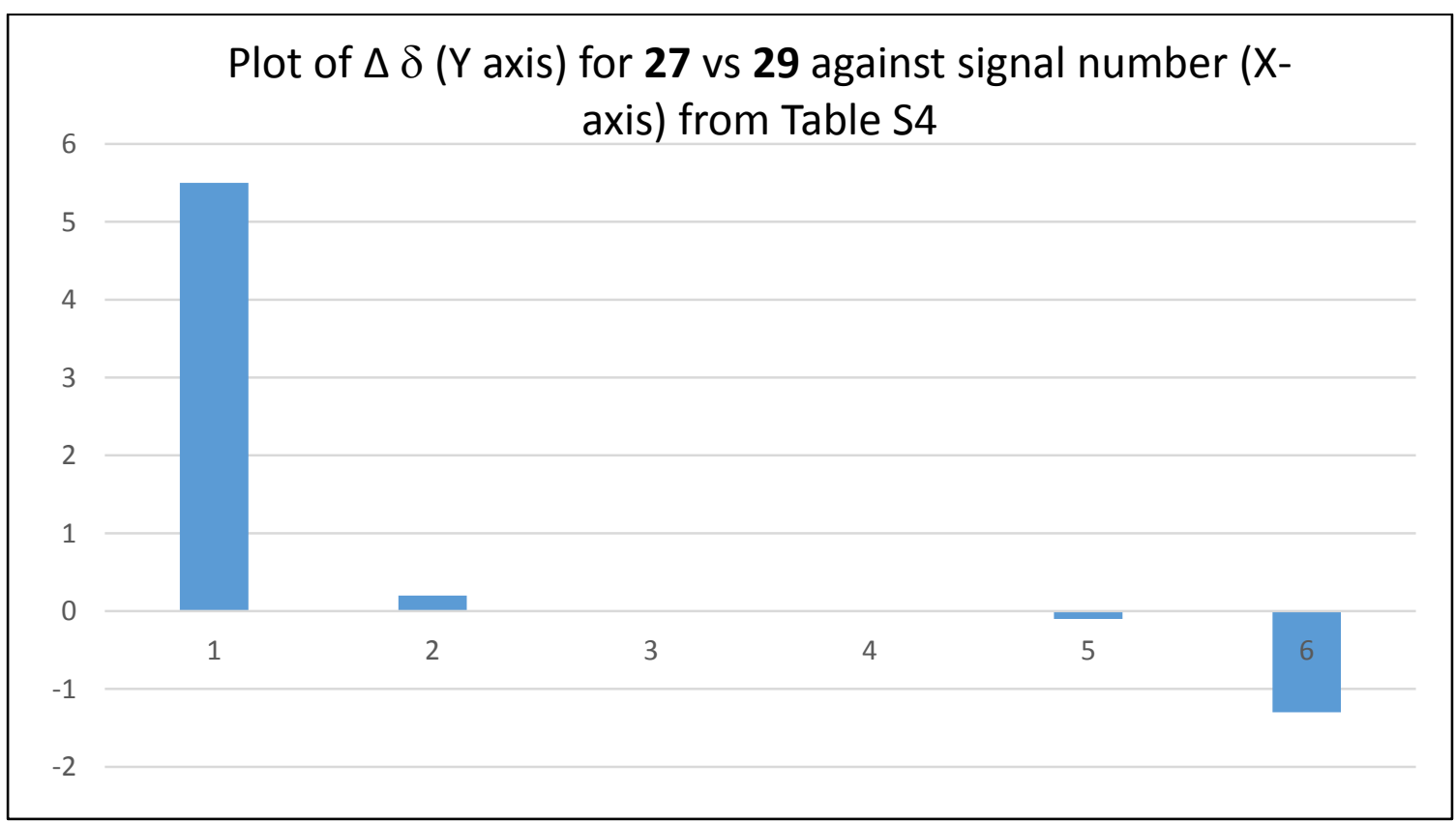


Supporting information - Experimental Section

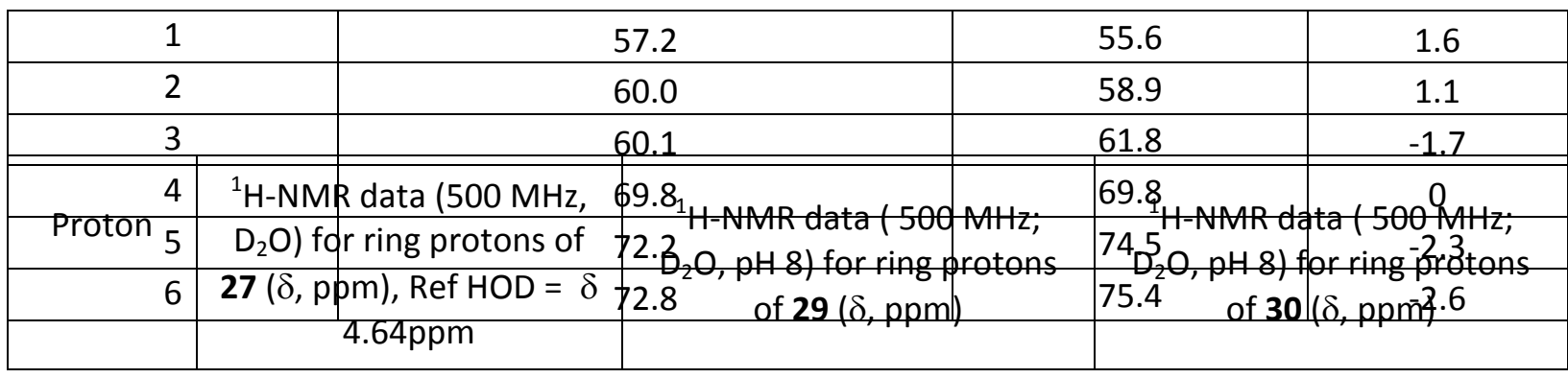

Table S5

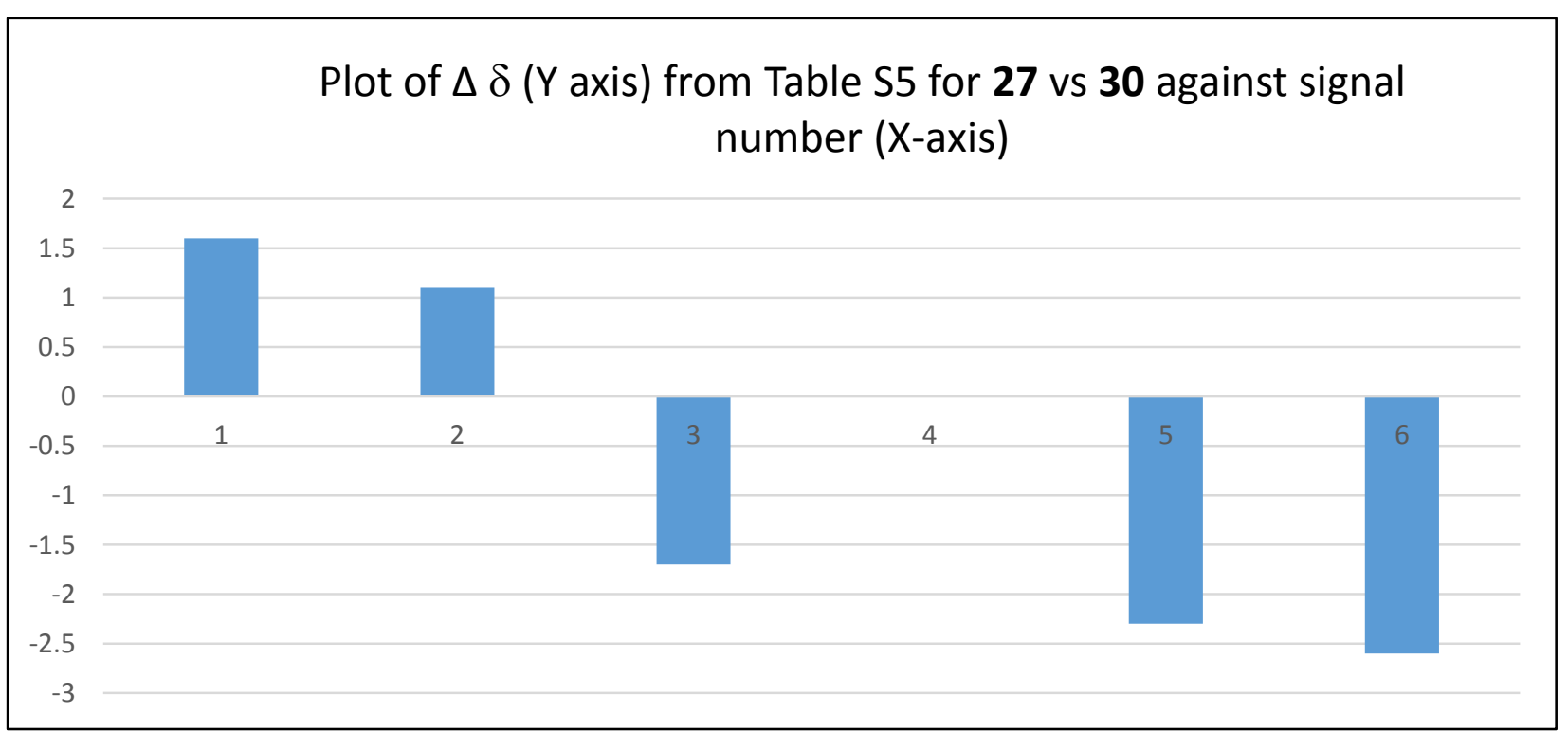

There is greater difference in Chemical shifts when comparing 27 with 30 than when comparing 27 with 29. 


\section{Supporting information - Experimental Section}

\begin{tabular}{|c|c|c|c|}
\hline $\mathrm{H}-1$ & $\begin{array}{l}\text { 2.55, ddd, } J=9.3,7.5 \\
3.9 \mathrm{~Hz}\end{array}$ & $2.59,1 \mathrm{H}, \mathrm{dq}, \mathrm{J} 9.4, \mathrm{~J} 6.3$ & $2.45, \mathrm{dq}, \mathrm{J} 9.6,6.4$ \\
\hline $\mathrm{H}-2$ & $3.43, \mathrm{t}, J=9.1 \mathrm{~Hz}$ & $3.26, \mathrm{dd}, \mathrm{J} 9.4, \mathrm{~J} 9.5$ & $3.21, \mathrm{dd}, \mathrm{J} 9.6,9.7$ \\
\hline $\mathrm{H}-3$ & $3.53, \mathrm{dd}, J=9.1,3.3$ & $3.51, \mathrm{dd}, \mathrm{J} 9.5,3.3$ & $3.40, \mathrm{dd}, \mathrm{J} 9.7,3.2$ \\
\hline $\mathrm{H}-4$ & $3.90, \mathrm{t}, J=3.1 \mathrm{~Hz}, 1 \mathrm{H}$ & $3.91, \mathrm{dd}, \mathrm{J} 2.3,3.3$ & $3.90, \mathrm{dd}, \mathrm{J} 1.3,3.2$ \\
\hline $\mathrm{H}-5$ & $\begin{array}{l}3.07 \text { (ddd, } J=7.7,7.2,2.7 \\
\mathrm{~Hz}, 1 \mathrm{H})\end{array}$ & $2.96, \mathrm{ddd}, \mathrm{J} 7.7,7.1,2.3$ & $2.73, \mathrm{ddd}, \mathrm{J} 6.9,6.7,1.3$ \\
\hline $\mathrm{H}-6$ & $\begin{array}{l}3.58, \mathrm{dd}, 11.8,7.2 \\
3.62 \mathrm{dd}, 11.8,7.7\end{array}$ & $\begin{array}{l}3.56, \mathrm{dd}, \mathrm{J} \text { 11.7, } 7.1 \\
3.62, \mathrm{dd}, \mathrm{J} 11.7,7.7\end{array}$ & $\begin{array}{l}3.49, \mathrm{dd}, \mathrm{J} 11.2,6.7 \\
3.53, \mathrm{dd}, \mathrm{J} 11.2,6.9\end{array}$ \\
\hline
\end{tabular}

Table S6 ${ }^{1}$ H-NMR data for 27 and comparison with 29/30

The source of ${ }^{1} \mathrm{H}-\mathrm{NMR}$ data for $\mathbf{2 9 / 3 0}$ is J. Chem. Soc., Perkin Trans. 1, 1999, 2735-2745

\section{References}

\footnotetext{
${ }^{1}$ Skaanderup, P. R.; Poulsen, C. S.; Hyldtoft, L.; Jørgensen, M. R.; Madsen, R., Synthesis 2002, 2002 (12), 1721-1727.

${ }^{2}$ Kumamoto, H.; Deguchi, K.; Wagata, T.; Furuya, Y.; Odanaka, Y.; Kitade, Y.; Tanaka, H., Tetrahedron 2009, 65 (38), 8007-8013.

${ }^{3}$ Long, D. D.; Smith, M. D.; Martin, A.; Wheatley, J. R.; Watkin, D. G.; Müller, M.; Fleet, G. W. J. J. Chem. Soc., Perkin Trans. 1, 2002, 1982.
} 